\title{
Linear statistics of matrix ensembles in classical background
}

\author{
Yang Chen and Chao Min \\ (yangbrookchen@yahoo.co.uk, chaominrunner@gmail.com) \\ Department of Mathematics, University of Macau, \\ Avenida da Universidade, Taipa, Macau, China
}

August 3, 2018

\begin{abstract}
Given a joint probability density function of $N$ real random variables, $\left\{x_{j}\right\}_{j=1}^{N}$, obtained from the eigenvector-eigenvalue decomposition of $N \times N$ random matrices, one constructs a random variable, the linear statistics, defined by the sum of smooth functions evaluated at the eigenvalues or singular values of the random matrix, namely, $\sum_{j=1}^{N} F\left(x_{j}\right)$.

For the jpdfs obtained from the Gaussian and Laguerre ensembles, we compute, in this paper the moment generating function $\mathbb{E}_{\beta}\left(\exp \left(-\lambda \sum_{j} F\left(x_{j}\right)\right)\right)$, where $\mathbb{E}_{\beta}$ denotes expectation value over the Orthogonal $(\beta=1)$ and Symplectic $(\beta=4)$ ensembles, in the form one plus a Schwartz function, none vanishing over $\mathbb{R}$ for the Gaussian ensembles and $\mathbb{R}^{+}$for the Laguerre ensembles.

These are ultimately expressed in the form of the determinants of identity plus a scalar operator, from which we obtained the large $N$ asymptotic of the linear statistics from suitably scaled $F(\cdot)$.
\end{abstract}

\section{Introduction}

The well-known joint probability density function for the eigenvalues $\left\{x_{j}\right\}_{j=1}^{N}$ of $N \times N$ Hermitian matrices from an orthogonal ensemble $(\beta=1)$, unitary ensemble $(\beta=2)$ or symplectic ensemble 
$(\beta=4)$ is given by 11

$$
P^{(\beta)}\left(x_{1}, x_{2}, \ldots, x_{N}\right) \prod_{j=1}^{N} d x_{j}=C_{N}^{(\beta)} \prod_{1 \leq j<k \leq N}\left|x_{j}-x_{k}\right|^{\beta} \prod_{j=1}^{N} w\left(x_{j}\right) d x_{j},
$$

where $w(x)$ is a weight function or a probability density supported on $[a, b]$. In this paper, we require that the moments of $w$, namely,

$$
s_{j}:=\int_{a}^{b} x^{j} w(x) d x
$$

to exist for $j=0,1,2, \ldots$ Here the normalization constant $C_{N}^{(\beta)}$ reads,

$$
C_{N}^{(\beta)}=\frac{1}{\int_{[a, b]^{N}} \prod_{1 \leq j<k \leq N}\left|x_{j}-x_{k}\right|^{\beta} \prod_{j=1}^{N} w\left(x_{j}\right) d x_{j}} .
$$

Many years ago, Selberg [12], obtained closed form expression for $C_{N}^{(\beta)}$, where $w(x)=x^{a}(1-x)^{b}, a>-1, b>-1, x \in[0,1]$, the Jacobi weight. The constant $C_{N}^{(\beta)}$ with the Gaussian weight $w(x)=\mathrm{e}^{-x^{2}}, x \in \mathbb{R}$ and Laguerre weight, $w(x)=x^{\alpha} \mathrm{e}^{-x}, \alpha>-1, \quad x \in \mathbb{R}^{+}$, can be found in [11].

If we take $w(x)=\mathrm{e}^{-x^{2}}, x \in \mathbb{R}$, these are known as the Gaussian orthogonal ensemble (GOE), Gaussian unitary ensemble (GUE) and Gaussian symplectic ensemble (GSE). If $w(x)=x^{\alpha} \mathrm{e}^{-x}, \quad \alpha>-1, \quad x \in \mathbb{R}^{+}$, then we have analogously the LOE, LUE and LSE.

The moment generating function,

$$
G_{N}^{(\beta)}(f):=\mathbb{E}_{\beta}\left(\mathrm{e}^{-\lambda \sum_{j=1}^{N} F\left(x_{j}\right)}\right):=\frac{\int_{[a, b]^{N}} \prod_{1 \leq j<k \leq N}\left|x_{j}-x_{k}\right|^{\beta} \prod_{j=1}^{N} w\left(x_{j}\right) \mathrm{e}^{-\lambda F\left(x_{j}\right)} d x_{j}}{\int_{[a, b]^{N}} \prod_{1 \leq j<k \leq N}\left|x_{j}-x_{k}\right|^{\beta} \prod_{j=1}^{N} w\left(x_{j}\right) d x_{j}},
$$

is given by

$$
G_{N}^{(\beta)}(f)=C_{N}^{(\beta)} \int_{[a, b]^{N}} \prod_{1 \leq j<k \leq N}\left|x_{j}-x_{k}\right|^{\beta} \prod_{j=1}^{N} w\left(x_{j}\right)\left[1+f\left(x_{j}\right)\right] d x_{j},
$$

where $\beta=1,2,4$. Here $\mathrm{e}^{-\lambda F(x)}:=1+f(x)$, and we do not indicate that $f(\cdot)$ also depends on $\lambda$. Throughout this paper, we assume $f(x)$ lies in the Schwartz space, and $1+f(x) \neq 0$ over $[a, b]$.

The simplest, very well-studied, unitary case corresponds to $\beta=2$, see [1, 4, 2, 3, 6, 15], and the references therein.

We state here, for later development, facts on orthogonal polynomials. Let $\varphi_{j}(x):=P_{j}(x) \sqrt{w(x)}, j=0,1,2, \ldots$, where $P_{j}(x)$ are the orthonormal polynomials of degree $j$ with respect to the weight $w(x)$ supported on $[a, b]$,

$$
\int_{a}^{b} P_{j}(x) P_{k}(x) w(x) d x=\delta_{j, k}, \quad j, k=0,1,2, \ldots
$$


It is a well-known fact that,

$$
G_{N}^{(2)}(f)=\operatorname{det}\left(I+K_{N}^{(2)} f\right)
$$

where $K_{N}^{(2)} f$ is an integral operator with kernel $K_{N}^{(2)}(x, y) f(y)$. Here $K_{N}^{(2)}(x, y):=\sum_{j=0}^{N-1} \varphi_{j}(x) \varphi_{j}(y)$, can be evaluated via the Christoffel-Darboux formula.

With Gaussian background, where $w(x)=\mathrm{e}^{-x^{2}}, x \in \mathbb{R}$, we have

$$
P_{j}(x):=\frac{H_{j}(x)}{c_{j}}, \quad c_{j}=\pi^{\frac{1}{4}} 2^{\frac{j}{2}} \sqrt{\Gamma(j+1)}, \quad j=0,1,2, \ldots
$$

where $H_{j}(x)$ are the Hermite polynomials of degree $j$.

With Laguerre background, where $w(x)=x^{\alpha} \mathrm{e}^{-x}, \alpha>-1, x \in \mathbb{R}^{+}$, we have

$$
P_{j}(x):=\frac{L_{j}^{(\alpha)}(x)}{c_{j}^{(\alpha)}}, \quad c_{j}^{(\alpha)}=\sqrt{\frac{\Gamma(j+\alpha+1)}{\Gamma(j+1)}}, j=0,1,2, \ldots,
$$

where $L_{j}^{(\alpha)}(x)$ are the Laguerre polynomials of degree $j$. Properties of the Hermite and Laguerre polynomials can be found in [13].

We shall mainly deal with the $\beta=4$ and $\beta=1$ cases, which are more complicated than $\beta=2$ situation, especially so for $\beta=1$.

We always begin with the general case, and then apply the results obtained to the two classical ensembles, i.e., the Gaussian ensembles and Laguerre ensembles.

Furthermore, in $\beta=1$ situation, for convenience, $N$ is taken to be even, and it is expeditious to make use of the square root of the Gaussian weight, $\mathrm{e}^{-x^{2} / 2}, x \in \mathbb{R}$ and the square root of the Laguerre weight, $x^{\alpha / 2} \mathrm{e}^{-x / 2}, \alpha>-2, x \in \mathbb{R}^{+}$in later discussion.

In this paper, we shall be concerned with the large $N$ behavior of $G_{N}^{(\beta)}(f)$ for the Gaussian ensembles and Laguerre ensembles. For the Gaussian ensembles, we replace $f(x)$ by $f(\sqrt{2 N} x)$, in the orthogonal case, and $f(\sqrt{4 N} x)$, in the symplectic case. For the Laguerre ensembles, we replace $f(x)$ by $f(\sqrt{4 N x})$, in the orthogonal case and $f(\sqrt{8 N x})$, in the symplectic case.

We will ultimately give the mean and variance of the linear statistics, as $N \rightarrow \infty$, together with leading correction terms.

For comparison purposes, we write down results in the GUE, where, $w(x)=\mathrm{e}^{-x^{2}}, x \in \mathbb{R}$. Denote by $\mu_{N}^{(G U E)}$ and $\mathcal{V}_{N}^{(G U E)}$ the mean and variance of the linear statistics $\sum_{j=1}^{N} F\left(\sqrt{2 N} x_{j}\right)$. It is shown in [3], that,

$$
\mu_{N}^{(G U E)} \rightarrow \frac{1}{\pi} \int_{-\infty}^{\infty} F(x) d x, \quad N \rightarrow \infty
$$




$$
\mathcal{V}_{N}^{(G U E)} \rightarrow \frac{1}{\pi} \int_{-\infty}^{\infty} F^{2}(x) d x-\int_{-\infty}^{\infty} \int_{-\infty}^{\infty}\left[\frac{\sin (x-y)}{\pi(x-y)}\right]^{2} F(x) F(y) d x d y, \quad N \rightarrow \infty .
$$

For the LUE, where $w(x)=x^{\alpha} \mathrm{e}^{-x}, \alpha>-1, x \in \mathbb{R}^{+}$. Denote by $\mu_{N}^{(L U E, \alpha)}$ and $\mathcal{V}_{N}^{(L U E, \alpha)}$ the mean and variance of the linear statistics $\sum_{j=1}^{N} F\left(\sqrt{4 N x_{j}}\right)$. It is shown in[2], that,

$$
\begin{gathered}
\mu_{N}^{(L U E, \alpha)} \rightarrow \int_{0}^{\infty} B^{(\alpha)}(x, x) F(x) d x, \quad N \rightarrow \infty \\
\mathcal{V}_{N}^{(L U E, \alpha)} \rightarrow \int_{0}^{\infty} B^{(\alpha)}(x, x) F^{2}(x) d x-\int_{0}^{\infty} \int_{0}^{\infty} B^{(\alpha)}(x, y) B^{(\alpha)}(y, x) F(x) F(y) d x d y, \quad N \rightarrow \infty
\end{gathered}
$$

where

$$
\begin{gathered}
B^{(\alpha)}(x, y):=\frac{J_{\alpha}(x) y J_{\alpha}^{\prime}(y)-J_{\alpha}(y) x J_{\alpha}^{\prime}(x)}{x^{2}-y^{2}} x, \\
B^{(\alpha)}(x, x):=\frac{J_{\alpha}^{2}(x)-J_{\alpha-1}(x) J_{\alpha+1}(x)}{2} x .
\end{gathered}
$$

Here $J_{\alpha}(\cdot)$ is the Bessel function of order $\alpha$.

We want to point out, the motivation of this paper comes from [6], which provided results both for symplectic ensembles and orthogonal ensembles, and specialize to the Gaussian case, i.e., GSE and GOE. The [6] dealt with the situation where $f($.$) is the characteristic function of an interval$ (or the union of disjoint intervals), and focus on the distribution of the $m$ th largest eigenvalue in the GSE and GOE, while we are interested for "smooth" $f$, and we also consider the Laguerre case, i.e., LSE and LOE.

This paper is organized as follows. In Section 2, we recall a number of theorems, the operators $D$ and $\varepsilon$, and end with two Lemmas relevant for later development. Section 3 begins with a general discussion of the Symplectic ensembles in a general setting, followed by detailed discussions on the GSE and LSE cases and ends with the computation of the mean and variance of linear statistics for large $N$. Section 4 repeats the development in Section 3 but for the Orthogonal ensembles, which is harder. We conclude in Section 5.

\section{Preliminaries}

For orientation purposes, we introduce here a number of results, which will be used throughout this paper. 
Theorem 2.1. The Stirling's formula [10]

$$
\Gamma(n)=\sqrt{2 \pi} n^{n-\frac{1}{2}} \mathrm{e}^{-n}\left[1+O\left(n^{-1}\right)\right], \quad n \rightarrow \infty .
$$

\section{Lemma 2.2.}

$$
{ }_{2} F_{1}\left(-N, 1 ; \frac{3}{2} ; 2\right)=(-1)^{N} \sqrt{\frac{\pi}{8 N}}+O\left(N^{-1}\right), \quad N \rightarrow \infty .
$$

Proof. From the integral representation of the hypergeometric function,

$$
{ }_{2} F_{1}(\alpha, \beta ; \gamma ; z)=\frac{\Gamma(\gamma)}{\Gamma(\beta) \Gamma(\gamma-\beta)} \int_{0}^{1} t^{\beta-1}(1-t)^{\gamma-\beta-1}(1-t z)^{-\alpha} d t, \quad \operatorname{Re} \gamma>\operatorname{Re} \beta>0,
$$

we obtain,

$$
{ }_{2} F_{1}\left(-N, 1 ; \frac{3}{2} ; 2\right)=\frac{1}{2} \int_{0}^{1} \frac{(1-2 t)^{N}}{\sqrt{1-t}} d t .
$$

Let

$$
x=\sqrt{1-t},
$$

then

$$
\begin{aligned}
{ }_{2} F_{1}\left(-N, 1 ; \frac{3}{2} ; 2\right) & =\int_{0}^{1}\left(2 x^{2}-1\right)^{N} d x \\
& =\int_{0}^{\frac{\sqrt{2}}{2}}\left(2 x^{2}-1\right)^{N} d x+\int_{\frac{\sqrt{2}}{2}}^{1}\left(2 x^{2}-1\right)^{N} d x \\
& =(-1)^{N} \int_{0}^{\frac{\sqrt{2}}{2}}\left(1-2 x^{2}\right)^{N} d x+\int_{\frac{\sqrt{2}}{2}}^{1}\left(2 x^{2}-1\right)^{N} d x .
\end{aligned}
$$

Consider the first integral in (2.3) $)$. Let

$$
x=\frac{\sqrt{2}}{2} \cos \theta, \quad \theta \in\left[0, \frac{\pi}{2}\right],
$$

then

$$
\begin{aligned}
\int_{0}^{\frac{\sqrt{2}}{2}}\left(1-2 x^{2}\right)^{N} d x & =\frac{\sqrt{2}}{2} \int_{0}^{\frac{\pi}{2}}(\sin \theta)^{2 N+1} d \theta \\
& =\frac{\sqrt{2}}{2} \frac{\left[2^{N} \Gamma(N+1)\right]^{2}}{\Gamma(2 N+2)} \\
& =\sqrt{\frac{\pi}{8 N}}\left[1+O\left(N^{-1}\right)\right], \quad N \rightarrow \infty,
\end{aligned}
$$

where use has been made of the Stirling's formula (2.1), in the last equality. 
Now consider the second integral in (2.3),

$$
\begin{aligned}
\int_{\frac{\sqrt{2}}{2}}^{1}\left(2 x^{2}-1\right)^{N} d x & \leq \int_{\frac{\sqrt{2}}{2}}^{1}(2 x-1)^{N} d x \\
& =\frac{1-(\sqrt{2}-1)^{N+1}}{2(N+1)} .
\end{aligned}
$$

Hence

$$
{ }_{2} F_{1}\left(-N, 1 ; \frac{3}{2} ; 2\right)=(-1)^{N} \sqrt{\frac{\pi}{8 N}}+O\left(N^{-1}\right), \quad N \rightarrow \infty .
$$

Lemma 2.3. If $\alpha>0$, then

$$
\sum_{m=0}^{2 n} \frac{(-1)^{m}}{m ! \prod_{l=m}^{2 n}(\alpha+2 l)}\left(\begin{array}{c}
2 n+\alpha \\
2 n-m+1
\end{array}\right)=\frac{1}{(2 n+1) !}, \quad n=0,1,2, \ldots,
$$

where $\left(\begin{array}{l}j \\ k\end{array}\right):=\frac{\Gamma(j+1)}{\Gamma(k+1) \Gamma(j-k+1)}$.

Proof. Firstly,

$$
\begin{aligned}
& \sum_{m=0}^{2 n} \frac{(-1)^{m}}{m ! \prod_{l=m}^{2 n}(\alpha+2 l)}\left(\begin{array}{c}
2 n+\alpha \\
2 n-m+1
\end{array}\right) \\
= & \frac{1}{(2 n+1) !}+\frac{\Gamma(2 n+\alpha+1) \Gamma\left(\frac{\alpha}{2}\right){ }_{2} F_{1}\left(-2 n-1, \frac{\alpha}{2} ; \alpha ; 2\right)}{2^{2 n+1} \Gamma(2 n+2) \Gamma\left(2 n+\frac{\alpha}{2}+1\right) \Gamma(\alpha)} .
\end{aligned}
$$

From (2.2), we see that,

$$
{ }_{2} F_{1}\left(-2 n-1, \frac{\alpha}{2} ; \alpha ; 2\right)=\frac{\Gamma(\alpha)}{\Gamma^{2}\left(\frac{\alpha}{2}\right)} \int_{0}^{1} t^{\frac{\alpha}{2}-1}(1-t)^{\frac{\alpha}{2}-1}(1-2 t)^{2 n+1} d t .
$$

Let

$$
t=\cos ^{2}\left(\frac{\theta}{2}\right), \quad \theta \in[0, \pi]
$$

then

$$
\int_{0}^{1} t^{\frac{\alpha}{2}-1}(1-t)^{\frac{\alpha}{2}-1}(1-2 t)^{2 n+1} d t=-\left(\frac{1}{2}\right)^{\alpha-1} \int_{0}^{\pi}(\sin \theta)^{\alpha-1}(\cos \theta)^{2 n+1} d \theta .
$$

We carry out mathematical induction in $n$ to prove that for $\alpha>0, \int_{0}^{\pi}(\sin \theta)^{\alpha-1}(\cos \theta)^{2 n+1} d \theta=0$. Setting if $n=0$, in the integral above, we have,

$$
\begin{aligned}
& \int_{0}^{\pi}(\sin \theta)^{\alpha-1} \cos \theta d \theta \\
= & \int_{0}^{\pi}(\sin \theta)^{\alpha-1} d \sin \theta \\
= & \left.\frac{(\sin \theta)^{\alpha}}{\alpha}\right|_{0} ^{\pi} \\
= & 0 .
\end{aligned}
$$


Suppose $\int_{0}^{\pi}(\sin \theta)^{\alpha-1}(\cos \theta)^{2 k+1} d \theta=0$, for $\alpha>0$, then

$$
\begin{aligned}
& \int_{0}^{\pi}(\sin \theta)^{\alpha-1}(\cos \theta)^{2 k+3} d \theta \\
= & \int_{0}^{\pi}(\sin \theta)^{\alpha-1}(\cos \theta)^{2 k+1} \cos ^{2} \theta d \theta \\
= & \int_{0}^{\pi}(\sin \theta)^{\alpha-1}(\cos \theta)^{2 k+1}\left(1-\sin ^{2} \theta\right) d \theta \\
= & \int_{0}^{\pi}(\sin \theta)^{\alpha-1}(\cos \theta)^{2 k+1} d \theta-\int_{0}^{\pi}(\sin \theta)^{\alpha+1}(\cos \theta)^{2 k+1} d \theta \\
= & 0 .
\end{aligned}
$$

Hence,

$$
\int_{0}^{\pi}(\sin \theta)^{\alpha-1}(\cos \theta)^{2 n+1} d \theta=0, \quad \alpha>0 .
$$

It follows that, for $\alpha>0$,

$$
{ }_{2} F_{1}\left(-2 n-1, \frac{\alpha}{2} ; \alpha ; 2\right)=0
$$

and from (2.4), the lemma follows.

\section{Theorem 2.4.}

$$
\prod_{1 \leq j<k \leq N}\left(x_{j}-x_{k}\right)^{4}=\operatorname{det}\left(x_{k}^{j}, j x_{k}^{j-1}\right)_{\substack{j=0, \ldots, 2 N-1 \\ k=1, \ldots, N}},
$$

where the determinant on the right is a $2 N \times 2 N$ determinant with alternating columns $\left\{x_{1}^{j}\right\},\left\{j x_{1}^{j-1}\right\},\left\{x_{2}^{j}\right\},\left\{j x_{2}^{j-1}\right\}, \ldots[9]$.

The next theorem, due to de Bruijn [5], is instrumental for the finite $N$ computations in Sections 3 and 4 .

Theorem 2.5. For any integrable functions $p_{j}(x)$ and $q_{j}(x), j=1,2, \ldots$, we have

$$
\begin{gathered}
\left(\int_{[a, b]^{N}} \operatorname{det}\left(p_{j}\left(x_{k}\right), q_{j}\left(x_{k}\right)\right)_{\substack{j=1, \ldots, 2 N \\
k=1, \ldots, N}} d x_{1} \cdots d x_{N}\right)^{2}=(N !)^{2} \operatorname{det}\left(\int_{a}^{b}\left(p_{j}(x) q_{k}(x)-p_{k}(x) q_{j}(x)\right) d x\right)_{j, k=1}^{2 N}, \\
\left(\int_{a \leq x_{1} \leq \cdots \leq x_{N} \leq b} \operatorname{det}\left(p_{j}\left(x_{k}\right)\right)_{j, k=1}^{N} d x_{1} \cdots d x_{N}\right)^{2}=\operatorname{det}\left(\int_{a}^{b} \int_{a}^{b} \operatorname{sgn}(y-x) p_{j}(x) p_{k}(y) d x d y\right)_{j, k=1}^{N},
\end{gathered}
$$

where the determinant on the left of the first equality is a $2 N \times 2 N$ determinant with alternating columns $\left\{p_{j}\left(x_{1}\right)\right\},\left\{q_{j}\left(x_{1}\right)\right\},\left\{p_{j}\left(x_{2}\right)\right\},\left\{q_{j}\left(x_{2}\right)\right\}, \ldots$ In addition, $N$ is even in the second equality. 
Theorem 2.6. If $A, B$ are Hilbert-Schmidt operators on a Hilbert space $\mathcal{H}$, then [7]

$$
\operatorname{det}(I+A B)=\operatorname{det}(I+B A) .
$$

Following [14, 15, 16], we introduce here operators $\varepsilon$ and $D$ which will be crucial for later development. Let $\varepsilon$ be the integral operator with kernel

$$
\varepsilon(x, y):=\frac{1}{2} \operatorname{sgn}(x-y),
$$

then for any integrable function $g$ defined on $[a, b]$,

$$
\varepsilon g(x)=\int_{a}^{b} \varepsilon(x, t) g(t) d t=\frac{1}{2}\left(\int_{a}^{x} g(t) d t-\int_{x}^{b} g(t) d t\right), \quad x \in[a, b] .
$$

It is clear that $\varepsilon(y, x)=-\varepsilon(x, y)$, i.e., $\varepsilon^{t}=-\varepsilon$, where ${ }^{t}$ denotes transpose.

Let $D$ be the operator that acts by differentiation, thus for any differentiable function $g$ defined on $[a, b]$,

$$
D g(x)=\frac{d g(x)}{d x}=g^{\prime}(x) .
$$

With further conditions on $g(x)$, we prove an easy lemma on the commutator $[D, \varepsilon]$.

Lemma 2.7. For any function $g \in C^{1}[a, b]$ and $g(a)=g(b)=0, D \varepsilon g(x)=\varepsilon D g(x)=g(x)$, i.e., $D \varepsilon=\varepsilon D=I$.

Proof. For any function $g \in C^{1}[a, b]$ and $g(a)=g(b)=0$, we have

$$
\begin{aligned}
(D \varepsilon) g(x) & =\frac{d}{d x} \int_{a}^{b} \varepsilon(x, t) g(t) d t \\
& =\frac{1}{2} \frac{d}{d x}\left[\int_{a}^{x} g(t) d t-\int_{x}^{b} g(t) d t\right] \\
& =\frac{1}{2} g(x)+\frac{1}{2} g(x)=g(x),
\end{aligned}
$$

and

$$
\begin{aligned}
(\varepsilon D) g(x) & =\int_{a}^{b} \varepsilon(x, t) g^{\prime}(t) d t \\
& =\frac{1}{2} \int_{a}^{x} g^{\prime}(t) d t-\frac{1}{2} \int_{x}^{b} g^{\prime}(t) d t \\
& =\frac{1}{2}[g(x)-g(a)]-\frac{1}{2}[g(b)-g(x)] \\
& =g(x)-\frac{1}{2}[g(a)+g(b)] \\
& =g(x) .
\end{aligned}
$$


The proof is complete.

Denote by $u \otimes v$ the integral operator with kernel $(u \otimes v)(x, y):=u(x) v(y)$. We have the following lemma.

Lemma 2.8. If $A$ is an integral operator with kernel $A(x, y)$, then

$$
A(u \otimes v)=(A u) \otimes v, \quad(u \otimes v) A=u \otimes\left(A^{t} v\right)
$$

\section{The symplectic ensembles}

\subsection{General case}

Taking $\beta=4$ in (1.1) gives the symplectic ensembles and generating function becomes,

$$
G_{N}^{(4)}(f)=C_{N}^{(4)} \int_{[a, b]^{N}} \prod_{1 \leq j<k \leq N}\left(x_{j}-x_{k}\right)^{4} \prod_{j=1}^{N} w\left(x_{j}\right)\left[1+f\left(x_{j}\right)\right] d x_{j}
$$

where

$$
C_{N}^{(4)}=\frac{1}{\int_{[a, b]^{N}} \prod_{1 \leq j<k \leq N}\left(x_{j}-x_{k}\right)^{4} \prod_{j=1}^{N} w\left(x_{j}\right) d x_{j}}
$$

is a constant depending on $N$.

We follow closely the computations of Dieng [6], and Tracy and Widom [15].

From Theorem 2.4 and Theorem 2.5 and some linear algebra, we get

$$
\left[G_{N}^{(4)}(f)\right]^{2}=\widehat{C_{N}^{(4)}} \operatorname{det}\left(\int_{a}^{b}\left[\pi_{j}(x) \pi_{k}^{\prime}(x)-\pi_{j}^{\prime}(x) \pi_{k}(x)\right] w(x)(1+f(x)) d x\right)_{j, k=0}^{2 N-1},
$$

where $\widehat{C_{N}^{(4)}}$ is a $N$ dependent constant, and $\pi_{j}(x)$ is any polynomial of degree $j$. Let

$$
\psi_{j}(x)=\pi_{j}(x) \sqrt{w(x)}
$$

and following [6, 15], we see that,

$$
\left[G_{N}^{(4)}(f)\right]^{2}=\operatorname{det}\left(I+\left(M^{(4)}\right)^{-1} L^{(4)}\right)
$$

where $M^{(4)}, L^{(4)}$ are matrices given by

$$
M^{(4)}=\left(\int_{a}^{b}\left(\psi_{j}(x) \psi_{k}^{\prime}(x)-\psi_{j}^{\prime}(x) \psi_{k}(x)\right) d x\right)_{j, k=0}^{2 N-1}
$$




$$
L^{(4)}=\left(\int_{a}^{b}\left(\psi_{j}(x) \psi_{k}^{\prime}(x)-\psi_{j}^{\prime}(x) \psi_{k}(x)\right) f(x) d x\right)_{j, k=0}^{2 N-1}
$$

With the notation,

$$
\left(M^{(4)}\right)^{-1}=:\left(\mu_{j k}\right)_{j, k=0}^{2 N-1}
$$

we obtain, finally,

$$
\left[G_{N}^{(4)}(f)\right]^{2}=\operatorname{det}\left(I+K_{N}^{(4)} f\right)
$$

where $K_{N}^{(4)}$ is the integral operator

$$
K_{N}^{(4)}=\left(\begin{array}{cc}
\sum_{j, k=0}^{2 N-1} \mu_{j k} \psi_{j}^{\prime} \otimes \psi_{k} & -\sum_{j, k=0}^{2 N-1} \mu_{j k} \psi_{j}^{\prime} \otimes \psi_{k}^{\prime} \\
\sum_{j, k=0}^{2 N-1} \mu_{j k} \psi_{j} \otimes \psi_{k} & -\sum_{j, k=0}^{2 N-1} \mu_{j k} \psi_{j} \otimes \psi_{k}^{\prime}
\end{array}\right)=:\left(\begin{array}{cc}
K_{N, 4}^{(1,1)} & K_{N, 4}^{(1,2)} \\
K_{N, 4}^{(2,1)} & K_{N, 4}^{(2,2)}
\end{array}\right)
$$

In the next theorem, we obtain further relations on $K_{N, 4}^{(i, j)}, i, j=1,2$, which ultimately expresses $K_{N}^{(4)}$ in terms of $K_{N, 4}^{(2,2)}$.

\section{Theorem 3.1.}

$$
K_{N, 4}^{(2,1)}=K_{N, 4}^{(2,2)} \varepsilon, \quad K_{N, 4}^{(1,1)}=D K_{N, 4}^{(2,2)} \varepsilon, \quad K_{N, 4}^{(1,2)}=D K_{N, 4}^{(2,2)}, \quad K_{N, 4}^{(2,2)} \varepsilon D=K_{N, 4}^{(2,2)}
$$

Proof. First of all,

$$
\begin{aligned}
K_{N, 4}^{(2,2)} \varepsilon & =-\sum_{j, k=0}^{2 N-1}\left(\mu_{j k} \psi_{j} \otimes \psi_{k}^{\prime}\right) \varepsilon \\
& =\sum_{j, k=0}^{2 N-1} \mu_{j k} \psi_{j} \otimes\left(\varepsilon \psi_{k}^{\prime}\right) \\
& =\sum_{j, k=0}^{2 N-1} \mu_{j k} \psi_{j} \otimes \psi_{k} \\
& =K_{N, 4}^{(2,1)}
\end{aligned}
$$

Secondly, for any integrable function $g(x)$ defined on $[a, b]$, we have

$$
\begin{aligned}
D K_{N, 4}^{(2,1)} g(x) & =\frac{d}{d x} \int_{a}^{b} K_{N, 4}^{(2,1)}(x, y) g(y) d y \\
& =\int_{a}^{b} \frac{\partial}{\partial x} K_{N, 4}^{(2,1)}(x, y) g(y) d y \\
& =\int_{a}^{b} K_{N, 4}^{(1,1)}(x, y) g(y) d y \\
& =K_{N, 4}^{(1,1)} g(x),
\end{aligned}
$$


i.e.,

$$
D K_{N, 4}^{(2,1)}=K_{N, 4}^{(1,1)} .
$$

It follows that

$$
K_{N, 4}^{(1,1)}=D K_{N, 4}^{(2,2)} \varepsilon .
$$

Similarly,

$$
\begin{aligned}
D K_{N, 4}^{(2,2)} g(x) & =\frac{d}{d x} \int_{a}^{b} K_{N, 4}^{(2,2)}(x, y) g(y) d y=\int_{a}^{b} \frac{\partial}{\partial x} K_{N, 4}^{(2,2)}(x, y) g(y) d y \\
& =\int_{a}^{b} K_{N, 4}^{(1,2)}(x, y) g(y) d y \\
& =K_{N, 4}^{(1,2)} g(x)
\end{aligned}
$$

i.e.,

$$
K_{N, 4}^{(1,2)}=D K_{N, 4}^{(2,2)} .
$$

Finally, we find

$$
\begin{aligned}
K_{N, 4}^{(2,2)} \varepsilon D g(x) & =K_{N, 4}^{(2,1)} D g(x)=\int_{a}^{b} K_{N, 4}^{(2,1)}(x, y) g^{\prime}(y) d y \\
& =\left.K_{N, 4}^{(2,1)}(x, y) g(y)\right|_{a} ^{b}-\int_{a}^{b} g(y) \frac{\partial}{\partial y} K_{N, 4}^{(2,1)}(x, y) d y
\end{aligned}
$$

Note that

$$
\begin{aligned}
K_{N, 4}^{(2,1)}(x, y): & =\sum_{j, k=0}^{2 N-1} \psi_{j}(x) \mu_{j k} \psi_{k}(y) \\
& =\sum_{j, k=0}^{2 N-1} \psi_{j}(x) \mu_{j k} \pi_{k}(y) \sqrt{w(y)}
\end{aligned}
$$

and

$$
w(a)=w(b)=0,
$$

hence

$$
K_{N, 4}^{(2,1)}(x, a)=K_{N, 4}^{(2,1)}(x, b)=0 .
$$

Continuing,

$$
\begin{aligned}
K_{N, 4}^{(2,2)} \varepsilon D g(x) & =-\int_{a}^{b} g(y) \frac{\partial}{\partial y} K_{N, 4}^{(2,1)}(x, y) d y=\int_{a}^{b} K_{N, 4}^{(2,2)}(x, y) g(y) d y \\
& =K_{N, 4}^{(2,2)} g(x)
\end{aligned}
$$


i.e.,

$$
K_{N, 4}^{(2,2)} \varepsilon D=K_{N, 4}^{(2,2)}
$$

The proof is complete.

According to Theorem 3.1, $K_{N}^{(4)}$ can be written as

$$
\begin{aligned}
K_{N}^{(4)} & =\left(\begin{array}{cc}
D K_{N, 4}^{(2,2)} \varepsilon & D K_{N, 4}^{(2,2)} \\
K_{N, 4}^{(2,2)} \varepsilon & K_{N, 4}^{(2,2)}
\end{array}\right)=\left(\begin{array}{ll}
D & 0 \\
0 & I
\end{array}\right)\left(\begin{array}{ll}
K_{N, 4}^{(2,2)} \varepsilon & K_{N, 4}^{(2,2)} \\
K_{N, 4}^{(2,2)} \varepsilon & K_{N, 4}^{(2,2)}
\end{array}\right) \\
& =: \widetilde{A} \widetilde{B}
\end{aligned}
$$

where

$$
\widetilde{A}=\left(\begin{array}{cc}
D & 0 \\
0 & I
\end{array}\right), \quad \widetilde{B}=\left(\begin{array}{ll}
K_{N, 4}^{(2,2)} \varepsilon & K_{N, 4}^{(2,2)} \\
K_{N, 4}^{(2,2)} \varepsilon & K_{N, 4}^{(2,2)}
\end{array}\right) .
$$

From (3.2) and using Theorem 2.6, we have

$$
\left[G_{N}^{(4)}(f)\right]^{2}=\operatorname{det}(I+(\widetilde{A} \widetilde{B}) f)=\operatorname{det}(I+\widetilde{A}(\widetilde{B} f))=\operatorname{det}(I+(\widetilde{B} f) \widetilde{A})=\operatorname{det}(I+\widetilde{B}(f \widetilde{A})) .
$$

Since

$$
\begin{aligned}
\widetilde{B}(f \widetilde{A}) & =\left(\begin{array}{ll}
K_{N, 4}^{(2,1)} & K_{N, 4}^{(2,2)} \\
K_{N, 4}^{(2,1)} & K_{N, 4}^{(2,2)}
\end{array}\right)\left(f\left(\begin{array}{ll}
D & 0 \\
0 & I
\end{array}\right)\right) \\
& =\left(\begin{array}{ll}
K_{N, 4}^{(2,2)} \varepsilon & K_{N, 4}^{(2,2)} \\
K_{N, 4}^{(2,2)} \varepsilon & K_{N, 4}^{(2,2)}
\end{array}\right)\left(\begin{array}{cc}
f D & 0 \\
0 & f
\end{array}\right) \\
& =\left(\begin{array}{ll}
K_{N, 4}^{(2,2)} \varepsilon f D & K_{N, 4}^{(2,2)} f \\
K_{N, 4}^{(2,2)} \varepsilon f D & K_{N, 4}^{(2,2)} f
\end{array}\right)
\end{aligned}
$$

then

$$
\left[G_{N}^{(4)}(f)\right]^{2}=\operatorname{det}\left(\begin{array}{cc}
I+K_{N, 4}^{(2,2)} \varepsilon f D & K_{N, 4}^{(2,2)} f \\
K_{N, 4}^{(2,2)} \varepsilon f D & I+K_{N, 4}^{(2,2)} f
\end{array}\right) .
$$

The computation below reduces the above into a determinant of scalar operators. We subtract row 1 from row 2 ,

$$
\left[G_{N}^{(4)}(f)\right]^{2}=\operatorname{det}\left(\begin{array}{cc}
I+K_{N, 4}^{(2,2)} \varepsilon f D & K_{N, 4}^{(2,2)} f \\
-I & I
\end{array}\right) .
$$


Next, add column 2 to column 1,

$$
\begin{aligned}
{\left[G_{N}^{(4)}(f)\right]^{2} } & =\operatorname{det}\left(\begin{array}{cc}
I+K_{N, 4}^{(2,2)} \varepsilon f D+K_{N, 4}^{(2,2)} f & K_{N, 4}^{(2,2)} f \\
0 & I
\end{array}\right) \\
& =\operatorname{det}\left(I+K_{N, 4}^{(2,2)} \varepsilon f D+K_{N, 4}^{(2,2)} f\right) .
\end{aligned}
$$

Remark. The above result agrees with [6] for the GUE case if we take $f=-\mu \chi_{J}$, where $\chi_{J}$ is the characteristic function of the interval $J$.

Now we use the commutator $[D, f]:=D f-f D$ to obtain a better suited result for our purpose. For a given function smooth $g(x)$, we have

$$
\begin{aligned}
{[D, f] g(x) } & =\operatorname{Dfg}(x)-f D g(x) \\
& =(f(x) g(x))^{\prime}-f(x) g^{\prime}(x) \\
& =f^{\prime}(x) g(x),
\end{aligned}
$$

this is,

$$
[D, f]=f^{\prime}
$$

It follows that

$$
f D=D f-[D, f]=D f-f^{\prime}
$$

Taking this into account, we have the following theorem.

\section{Theorem 3.2.}

$$
\left[G_{N}^{(4)}(f)\right]^{2}=\operatorname{det}\left(I+2 K_{N, 4}^{(2,2)} f-K_{N, 4}^{(2,2)} \varepsilon f^{\prime}\right)
$$

where the kernel of $K_{N, 4}^{(2,2)}$ reads,

$$
K_{N, 4}^{(2,2)}(x, y)=-\sum_{j, k=0}^{2 N-1} \psi_{j}(x) \mu_{j k} \psi_{k}^{\prime}(y)
$$

\subsection{GSE}

In the case of the Gaussian weight $w(x)=\mathrm{e}^{-x^{2}}, \quad x \in \mathbb{R}$, we again follow the discussions [6, 15], and choose a special $\psi_{j}$ to simplify $M^{(4)}$ as much as possible. To proceed, let

$$
\psi_{2 j+1}(x):=\frac{1}{\sqrt{2}} \varphi_{2 j+1}(x), \quad \psi_{2 j}(x):=-\frac{1}{\sqrt{2}} \varepsilon \varphi_{2 j+1}(x), \quad j=0,1,2, \ldots,
$$


where $\varphi_{j}(x)$ is given by

$$
\varphi_{j}(x)=\frac{H_{j}(x)}{c_{j}} \mathrm{e}^{-\frac{x^{2}}{2}}, \quad c_{j}=\pi^{\frac{1}{4}} 2^{\frac{j}{2}} \sqrt{\Gamma(j+1)},
$$

and $H_{j}(x), j=0,1, \ldots$ are the usual Hermite polynomials with the orthogonality condition

$$
\int_{-\infty}^{\infty} H_{m}(x) H_{n}(x) \mathrm{e}^{-x^{2}} d x=c_{n}^{2} \delta_{m n}
$$

We show in the next lemma that, this definition satisfies (3.1), i.e., $\psi_{j}(x)=\pi_{j}(x) \mathrm{e}^{-\frac{x^{2}}{2}}, j=0,1,2, \ldots$, where $\pi_{j}(x)$ is a polynomial of degree $j$.

Lemma 3.3. $\psi_{j}(x) \mathrm{e}^{\frac{x^{2}}{2}}, j=0,1,2, \ldots$ is a polynomial of degree $j$.

Proof. If the index is odd, then it is clear that $\psi_{2 j+1}(x) \mathrm{e}^{\frac{x^{2}}{2}}$ is a polynomial of degree $2 j+1$. For even index,

$$
\begin{aligned}
\psi_{2 j}(x) \mathrm{e}^{\frac{x^{2}}{2}} & =-\frac{1}{\sqrt{2}}\left(\varepsilon \varphi_{2 j+1}(x)\right) \mathrm{e}^{\frac{x^{2}}{2}} \\
& =-\frac{1}{\sqrt{2}} \mathrm{e}^{\frac{x^{2}}{2}} \varepsilon\left(\frac{1}{c_{2 j+1}} H_{2 j+1}(x) \mathrm{e}^{-\frac{x^{2}}{2}}\right) \\
& =-\frac{1}{2 \sqrt{2} c_{2 j+1}} \mathrm{e}^{\frac{x^{2}}{2}}\left[\int_{-\infty}^{x} H_{2 j+1}(y) \mathrm{e}^{-\frac{y^{2}}{2}} d y-\int_{x}^{\infty} H_{2 j+1}(y) \mathrm{e}^{-\frac{y^{2}}{2}} d y\right], j=0,1,2, \cdots
\end{aligned}
$$

Since $H_{2 j+1}(y) \mathrm{e}^{-\frac{y^{2}}{2}}, j=0,1, \ldots$ is an odd function, we have

$$
0=\int_{-\infty}^{\infty} H_{2 j+1}(y) \mathrm{e}^{-\frac{y^{2}}{2}} d y=\int_{-\infty}^{x} H_{2 j+1}(y) \mathrm{e}^{-\frac{y^{2}}{2}} d y+\int_{x}^{\infty} H_{2 j+1}(y) \mathrm{e}^{-\frac{y^{2}}{2}} d y
$$

Hence

$$
\int_{x}^{\infty} H_{2 j+1}(y) \mathrm{e}^{-\frac{y^{2}}{2}} d y=-\int_{-\infty}^{x} H_{2 j+1}(y) \mathrm{e}^{-\frac{y^{2}}{2}} d y
$$

and we find,

$$
\psi_{2 j}(x) \mathrm{e}^{\frac{x^{2}}{2}}=-\frac{1}{\sqrt{2} c_{2 j+1}} \mathrm{e}^{\frac{x^{2}}{2}} \int_{-\infty}^{x} H_{2 j+1}(y) \mathrm{e}^{-\frac{y^{2}}{2}} d y .
$$

From mathematical induction, it follows that

$$
\begin{aligned}
\int_{-\infty}^{x} y^{k} \mathrm{e}^{-\frac{y^{2}}{2}} d y & =-\mathrm{e}^{-\frac{x^{2}}{2}}\left[x^{k-1}+(k-1) x^{k-3}+(k-1)(k-3) x^{k-5}\right. \\
& +\cdots+(k-1)(k-3) \cdots 2], \quad k=1,3,5, \cdots
\end{aligned}
$$

Since $H_{2 j+1}(y)$ is a linear combination of $y, y^{3}, \ldots, y^{2 j+1}$, we see that $\int_{-\infty}^{x} H_{2 j+1}(y) \mathrm{e}^{-\frac{y^{2}}{2}} d y$ is equal to $\mathrm{e}^{-\frac{x^{2}}{2}}$ multiplying a polynomial of degree $2 j$. It follows that $\psi_{2 j}(x) \mathrm{e}^{\frac{x^{2}}{2}}$ is a polynomial of degree $2 j$. The proof is complete. 
Using (3.4) to compute

$$
M^{(4)}:=\left(\int_{-\infty}^{\infty}\left(\psi_{j}(x) \psi_{k}^{\prime}(x)-\psi_{j}^{\prime}(x) \psi_{k}(x)\right) d x\right)_{j, k=0}^{2 N-1}
$$

we obtain the following lemma.

\section{Lemma 3.4. (Dieng, Tracy - Widom)}

$$
M^{(4)}=\left(\begin{array}{ccccccc}
0 & 1 & 0 & 0 & \cdots & 0 & 0 \\
-1 & 0 & 0 & 0 & \cdots & 0 & 0 \\
0 & 0 & 0 & 1 & \cdots & 0 & 0 \\
0 & 0 & -1 & 0 & \cdots & 0 & 0 \\
\vdots & \vdots & \vdots & \vdots & & \vdots & \vdots \\
0 & 0 & 0 & 0 & \cdots & 0 & 1 \\
0 & 0 & 0 & 0 & \cdots & -1 & 0
\end{array}\right)_{2 N \times 2 N}
$$

It is clear that $\left(M^{(4)}\right)^{-1}=-M^{(4)}$, so $\mu_{2 j, 2 j+1}=-1, \mu_{2 j+1,2 j}=1$, and $\mu_{j k}=0$ for other cases. Hence

$$
\begin{aligned}
K_{N, 4}^{(2,2)}(x, y) & =-\sum_{j, k=0}^{2 N-1} \psi_{j}(x) \mu_{j k} \psi_{k}^{\prime}(y) \\
& =\sum_{j=0}^{N-1} \psi_{2 j}(x) \psi_{2 j+1}^{\prime}(y)-\sum_{j=0}^{N-1} \psi_{2 j+1}(x) \psi_{2 j}^{\prime}(y) \\
& =\frac{1}{2}\left[\sum_{j=0}^{N-1} \varphi_{2 j+1}(x) \varphi_{2 j+1}(y)-\sum_{j=0}^{N-1} \varepsilon \varphi_{2 j+1}(x) \varphi_{2 j+1}^{\prime}(y)\right] .
\end{aligned}
$$

Recall that the Hermite polynomials $H_{j}$ satisfy the differentiation formulas [10]

$$
\begin{gathered}
H_{j}^{\prime}(x)=2 x H_{j}(x)-H_{j+1}(x), \quad j=0,1,2, \ldots, \\
H_{j}^{\prime}(x)=2 j H_{j-1}(x), \quad j=0,1,2, \ldots
\end{gathered}
$$

Using the fact that $H_{j}(x)=c_{j} \varphi_{j}(x) \mathrm{e}^{\frac{x^{2}}{2}},(3.7)$ becomes

$$
\varphi_{j}^{\prime}(x)=x \varphi_{j}(x)-\sqrt{2(j+1)} \varphi_{j+1}(x), \quad j=0,1,2, \ldots,
$$

and similarly, (3.8) becomes,

$$
\varphi_{j}^{\prime}(x)=-x \varphi_{j}(x)+\sqrt{2 j} \varphi_{j-1}(x), \quad j=0,1,2, \ldots
$$


Combining (3.9) and (3.10), to eliminate $\varphi_{j}(x)$, we obtain

$$
\varphi_{j}^{\prime}(x)=\sqrt{\frac{j}{2}} \varphi_{j-1}(x)-\sqrt{\frac{j}{2}+\frac{1}{2}} \varphi_{j+1}(x), \quad j=0,1,2, \ldots
$$

Using (3.11) to replace $\varphi_{2 j+1}^{\prime}(y)$, we find,

$$
\begin{aligned}
& \sum_{j=0}^{N-1} \varepsilon \varphi_{2 j+1}(x) \varphi_{2 j+1}^{\prime}(y) \\
= & \sum_{j=0}^{N-1} \varepsilon \varphi_{2 j+1}(x)\left[\sqrt{j+\frac{1}{2}} \varphi_{2 j}(y)-\sqrt{j+1} \varphi_{2 j+2}(y)\right] \\
= & \sum_{j=0}^{N-1} \sqrt{j+\frac{1}{2}} \varepsilon \varphi_{2 j+1}(x) \varphi_{2 j}(y)-\sum_{j=0}^{N-1} \sqrt{j+1} \varepsilon \varphi_{2 j+1}(x) \varphi_{2 j+2}(y) \\
= & \sum_{j=0}^{N} \sqrt{j+\frac{1}{2}} \varepsilon \varphi_{2 j+1}(x) \varphi_{2 j}(y)-\sum_{j=0}^{N} \sqrt{j} \varepsilon \varphi_{2 j-1}(x) \varphi_{2 j}(y)-\sqrt{N+\frac{1}{2}} \varepsilon \varphi_{2 N+1}(x) \varphi_{2 N}(y) \\
= & -\sum_{j=0}^{N}\left[\sqrt{j} \varepsilon \varphi_{2 j-1}(x)-\sqrt{j+\frac{1}{2}} \varepsilon \varphi_{2 j+1}(x)\right] \varphi_{2 j}(y)-\sqrt{N+\frac{1}{2}} \varepsilon \varphi_{2 N+1}(x) \varphi_{2 N}(y)
\end{aligned}
$$

To proceed further, using Lemma 2.7, together with (3.11) we find

$$
\begin{aligned}
\varphi_{2 j}(x) & =\varepsilon D \varphi_{2 j}(x)=\varepsilon \varphi_{2 j}^{\prime}(x) \\
& =\sqrt{j} \varepsilon \varphi_{2 j-1}(x)-\sqrt{j+\frac{1}{2}} \varepsilon \varphi_{2 j+1}(x) .
\end{aligned}
$$

Substituting (3.13) into (3.12), it follows that

$$
\sum_{j=0}^{N-1} \varepsilon \varphi_{2 j+1}(x) \varphi_{2 j+1}^{\prime}(y)=-\sum_{j=0}^{N} \varphi_{2 j}(x) \varphi_{2 j}(y)-\sqrt{N+\frac{1}{2}} \varepsilon \varphi_{2 N+1}(x) \varphi_{2 N}(y) .
$$

Hence, (3.6) becomes,

$$
\begin{aligned}
K_{N, 4}^{(2,2)}(x, y) & =\frac{1}{2}\left[\sum_{j=0}^{N-1} \varphi_{2 j+1}(x) \varphi_{2 j+1}(y)+\sum_{j=0}^{N} \varphi_{2 j}(x) \varphi_{2 j}(y)+\sqrt{N+\frac{1}{2}} \varepsilon \varphi_{2 N+1}(x) \varphi_{2 N}(y)\right] \\
& =\frac{1}{2}\left[\sum_{j=0}^{2 N} \varphi_{j}(x) \varphi_{j}(y)+\sqrt{N+\frac{1}{2}} \varepsilon \varphi_{2 N+1}(x) \varphi_{2 N}(y)\right] \\
& =\frac{1}{2}\left[S_{N}(x, y)+\sqrt{N+\frac{1}{2}} \varepsilon \varphi_{2 N+1}(x) \varphi_{2 N}(y)\right],
\end{aligned}
$$

where

$$
S_{N}(x, y):=\sum_{j=0}^{2 N} \varphi_{j}(x) \varphi_{j}(y)=\sqrt{N+\frac{1}{2}} \frac{\varphi_{2 N+1}(x) \varphi_{2 N}(y)-\varphi_{2 N+1}(y) \varphi_{2 N}(x)}{x-y}
$$

and here the last equality comes from the Christoffel-Darboux formula.

By Theorem 3.2 , we have the following theorem. 


\section{Theorem 3.5.}

$$
\left[G_{N}^{(4)}(f)\right]^{2}=\operatorname{det}\left(I+S_{N} f-\frac{1}{2} S_{N} \varepsilon f^{\prime}+\sqrt{N+\frac{1}{2}}\left(\varepsilon \varphi_{2 N+1} \otimes \varphi_{2 N} f\right)+\frac{1}{2} \sqrt{N+\frac{1}{2}}\left(\varepsilon \varphi_{2 N+1} \otimes \varepsilon \varphi_{2 N}\right) f^{\prime}\right)
$$

\subsection{Large $N$ behavior of the GSE moment generating function}

To proceed with the large $N$ investigation, write, $\left[G_{N}^{(4)}(f)\right]^{2}$, as

$$
\left[G_{N}^{(4)}(f)\right]^{2}=: \operatorname{det}(I+T)
$$

where

$$
T:=S_{N} f-\frac{1}{2} S_{N} \varepsilon f^{\prime}+\sqrt{N+\frac{1}{2}}\left(\varepsilon \varphi_{2 N+1} \otimes \varphi_{2 N} f\right)+\frac{1}{2} \sqrt{N+\frac{1}{2}}\left(\varepsilon \varphi_{2 N+1} \otimes \varepsilon \varphi_{2 N}\right) f^{\prime}
$$

We find,

$$
\log \operatorname{det}(I+T)=\operatorname{Tr} \log (I+T)=\operatorname{Tr} T-\frac{1}{2} \operatorname{Tr} T^{2}+\frac{1}{3} \operatorname{Tr} T^{3}-\cdots
$$

This is obtained by the trace-log expansion of $\log \operatorname{det}(I+\nu T)$, where $0<\nu<1$, follow by a continuation to $\nu=1$. We assume that similar continuation also holds in other cases.

\section{Theorem 3.6.}

$$
\begin{gathered}
\lim _{N \rightarrow \infty} \frac{1}{\sqrt{4 N}} S_{N}\left(\frac{x}{\sqrt{4 N}}, \frac{y}{\sqrt{4 N}}\right)=\frac{\sin (x-y)}{\pi(x-y)}, \\
\lim _{N \rightarrow \infty} \frac{1}{\sqrt{4 N}} S_{N}\left(\frac{x}{\sqrt{4 N}}, \frac{x}{\sqrt{4 N}}\right)=\frac{1}{\pi} .
\end{gathered}
$$

The next theorem characterizes the large $N$ asymptotic of various "scaled" quantities.

\section{Theorem 3.7.}

$$
\begin{gathered}
\varphi_{2 N}\left(\frac{x}{\sqrt{4 N}}\right)=(-1)^{N} \pi^{-\frac{1}{2}} N^{-\frac{1}{4}} \cos x+O\left(N^{-\frac{3}{4}}\right), N \rightarrow \infty \\
\varepsilon \varphi_{2 N}\left(\frac{x}{\sqrt{4 N}}\right)=2^{-1}(-1)^{N} \pi^{-\frac{1}{2}} N^{-\frac{3}{4}} \sin x+O\left(N^{-\frac{5}{4}}\right), \quad N \rightarrow \infty \\
\varepsilon \varphi_{2 N+1}\left(\frac{x}{\sqrt{4 N}}\right)=-2^{-\frac{1}{2}} N^{-\frac{1}{4}}+O\left(N^{-\frac{3}{4}}\right), \quad N \rightarrow \infty .
\end{gathered}
$$


Proof. From the asymptotic expansion of Hermite polynomials [13],

$$
H_{n}(x) \mathrm{e}^{-\frac{x^{2}}{2}}=\lambda_{n}\left[\cos \left(\sqrt{2 n+1} x-\frac{n \pi}{2}\right)+O\left(n^{-\frac{1}{2}}\right)\right], n \rightarrow \infty,
$$

where

$$
\lambda_{2 n}=\frac{\Gamma(2 n+1)}{\Gamma(n+1)} \quad \text { and } \quad \lambda_{2 n+1}=\frac{\Gamma(2 n+3)}{\Gamma(n+2)}(4 n+3)^{-\frac{1}{2}} .
$$

We have

$$
\begin{aligned}
\varphi_{2 N}\left(\frac{x}{\sqrt{4 N}}\right) & =\frac{1}{\pi^{\frac{1}{4}} 2^{N} \sqrt{\Gamma(2 N+1)}} H_{2 N}\left(\frac{x}{\sqrt{4 N}}\right) \mathrm{e}^{-\frac{x^{2}}{8 N}} \\
& =\frac{1}{\pi^{\frac{1}{4}} 2^{N} \sqrt{\Gamma(2 N+1)}} \cdot \frac{\Gamma(2 N+1)}{\Gamma(N+1)}\left[\cos \left(\sqrt{\frac{4 N+1}{4 N}} x-N \pi\right)+O\left(N^{-\frac{1}{2}}\right)\right] \\
& =\frac{1}{\pi^{\frac{1}{4}} 2^{N}} \cdot \frac{\sqrt{\Gamma(2 N+1)}}{\Gamma(N+1)}\left[\cos (x-N \pi)+O\left(N^{-\frac{1}{2}}\right)\right] \\
& =(-1)^{N} \pi^{-\frac{1}{2}} N^{-\frac{1}{4}} \cos x+O\left(N^{-\frac{3}{4}}\right), \quad N \rightarrow \infty,
\end{aligned}
$$

where we have used the Stirling's formula (2.1).

A straightforward computation gives,

$$
\begin{aligned}
\varepsilon \varphi_{2 N}(x) & =\frac{1}{2}\left(\int_{-\infty}^{x} \varphi_{2 N}(y) d y-\int_{x}^{\infty} \varphi_{2 N}(y) d y\right) \\
& =\frac{1}{2}\left(\int_{-\infty}^{0} \varphi_{2 N}(y) d y+\int_{0}^{x} \varphi_{2 N}(y) d y-\int_{x}^{0} \varphi_{2 N}(y) d y-\int_{0}^{\infty} \varphi_{2 N}(y) d y\right) \\
& =\frac{1}{2}\left(2 \int_{0}^{x} \varphi_{2 N}(y) d y+\int_{-\infty}^{0} \varphi_{2 N}(y) d y-\int_{0}^{\infty} \varphi_{2 N}(y) d y\right) \\
& =\frac{1}{\pi^{\frac{1}{4}} 2^{N+1} \sqrt{\Gamma(2 N+1)}}\left(2 \int_{0}^{x} H_{2 N}(y) \mathrm{e}^{-\frac{y^{2}}{2}} d y+\int_{-\infty}^{0} H_{2 N}(y) \mathrm{e}^{-\frac{y^{2}}{2}} d y-\int_{0}^{\infty} H_{2 N}(y) \mathrm{e}^{-\frac{y^{2}}{2}} d y\right) \\
& =\frac{1}{\pi^{\frac{1}{4}} 2^{N} \sqrt{\Gamma(2 N+1)}} \int_{0}^{x} H_{2 N}(y) \mathrm{e}^{-\frac{y^{2}}{2}} d y .
\end{aligned}
$$

So we find, for large $N$,

$$
\begin{aligned}
\varepsilon \varphi_{2 N}\left(\frac{x}{\sqrt{4 N}}\right) & =\frac{1}{\pi^{\frac{1}{4}} 2^{N} \sqrt{\Gamma(2 N+1)}} \int_{0}^{\frac{x}{\sqrt{4 N}}} H_{2 N}(y) \mathrm{e}^{-\frac{y^{2}}{2}} d y \\
& =\frac{1}{\pi^{\frac{1}{4}} 2^{N} \sqrt{\Gamma(2 N+1)}} \cdot \frac{1}{\sqrt{4 N}} \int_{0}^{x} H_{2 N}\left(\frac{y}{\sqrt{4 N}}\right) \mathrm{e}^{-\frac{y^{2}}{8 N}} d y \\
& =\frac{1}{\pi^{\frac{1}{4}} 2^{N} \sqrt{\Gamma(2 N+1)}} \cdot \frac{1}{\sqrt{4 N}} \cdot \frac{\Gamma(2 N+1)}{\Gamma(N+1)} \int_{0}^{x}\left[(-1)^{N} \cos y+O\left(N^{-\frac{1}{2}}\right)\right] d y \\
& =\frac{\sqrt{\Gamma(2 N+1)}}{\pi^{\frac{1}{4}} 2^{N} \sqrt{4 N} \Gamma(N+1)}\left[(-1)^{N} \sin x+O\left(N^{-\frac{1}{2}}\right)\right] \\
& =2^{-1}(-1)^{N} \pi^{-\frac{1}{2}} N^{-\frac{3}{4}} \sin x+O\left(N^{-\frac{5}{4}}\right), \quad N \rightarrow \infty,
\end{aligned}
$$


where we have used the Stirling's formula (2.1) in the last step.

Finally, a straightforward computation gives,

$$
\begin{aligned}
\varepsilon \varphi_{2 N+1}(x) & =\frac{1}{2}\left[\int_{-\infty}^{x} \varphi_{2 N+1}(y) d y-\int_{x}^{\infty} \varphi_{2 N+1}(y) d y\right] \\
& =\frac{1}{\pi^{\frac{1}{4}} 2^{N+\frac{3}{2}} \sqrt{\Gamma(2 N+2)}}\left[\int_{-\infty}^{x} H_{2 N+1}(y) \mathrm{e}^{-\frac{y^{2}}{2}} d y-\int_{x}^{\infty} H_{2 N+1}(y) \mathrm{e}^{-\frac{y^{2}}{2}} d y\right] \\
& =\frac{1}{\pi^{\frac{1}{4}} 2^{N+\frac{3}{2}} \sqrt{\Gamma(2 N+2)}}\left[\int_{-\infty}^{0} H_{2 N+1}(y) \mathrm{e}^{-\frac{y^{2}}{2}} d y+\int_{0}^{x} H_{2 N+1}(y) \mathrm{e}^{-\frac{y^{2}}{2}} d y\right. \\
& \left.-\int_{x}^{0} H_{2 N+1}(y) \mathrm{e}^{-\frac{y^{2}}{2}} d y-\int_{0}^{\infty} H_{2 N+1}(y) \mathrm{e}^{-\frac{y^{2}}{2}} d y\right] \\
& =\frac{1}{\pi^{\frac{1}{4}} 2^{N+\frac{3}{2}} \sqrt{\Gamma(2 N+2)}}\left[2 \int_{0}^{x} H_{2 N+1}(y) \mathrm{e}^{-\frac{y^{2}}{2}} d y-2 \int_{0}^{\infty} H_{2 N+1}(y) \mathrm{e}^{-\frac{y^{2}}{2}} d y\right] \\
& =\frac{1}{\pi^{\frac{1}{4}} 2^{N+\frac{1}{2}} \sqrt{\Gamma(2 N+2)}}\left[\int_{0}^{x} H_{2 N+1}(y) \mathrm{e}^{-\frac{y^{2}}{2}} d y-\int_{0}^{\infty} H_{2 N+1}(y) \mathrm{e}^{-\frac{y^{2}}{2}} d y\right],
\end{aligned}
$$

continuing, we see that,

$$
\begin{aligned}
\varepsilon \varphi_{2 N+1}\left(\frac{x}{\sqrt{4 N}}\right) & =\frac{1}{\pi^{\frac{1}{4}} 2^{N+\frac{1}{2}} \sqrt{\Gamma(2 N+2)}} \int_{0}^{\frac{x}{\sqrt{4 N}}} H_{2 N+1}(y) \mathrm{e}^{-\frac{y^{2}}{2}} d y \\
& -\frac{1}{\pi^{\frac{1}{4}} 2^{N+\frac{1}{2}} \sqrt{\Gamma(2 N+2)}} \int_{0}^{\infty} H_{2 N+1}(y) \mathrm{e}^{-\frac{y^{2}}{2}} d y \\
& =\frac{1}{\pi^{\frac{1}{4}} 2^{N+\frac{1}{2}} \sqrt{\Gamma(2 N+2)}} \cdot \frac{1}{\sqrt{4 N}} \int_{0}^{x} H_{2 N+1}\left(\frac{y}{\sqrt{4 N}}\right) \mathrm{e}^{-\frac{y^{2}}{8 N}} d y \\
& -\frac{1}{\pi^{\frac{1}{4}} 2^{N+\frac{1}{2}} \sqrt{\Gamma(2 N+2)}} \int_{0}^{\infty} H_{2 N+1}(y) \mathrm{e}^{-\frac{y^{2}}{2}} d y .
\end{aligned}
$$

By (3.15), we have

$$
H_{2 N+1}\left(\frac{y}{\sqrt{4 N}}\right) \mathrm{e}^{-\frac{y^{2}}{8 N}}=\frac{\Gamma(2 N+3)}{\Gamma(N+2)}(4 N+3)^{-\frac{1}{2}}\left[(-1)^{N} \sin y+O\left(N^{-\frac{1}{2}}\right)\right], \quad N \rightarrow \infty .
$$

It follows that

$$
\int_{0}^{x} H_{2 N+1}\left(\frac{y}{\sqrt{4 N}}\right) \mathrm{e}^{-\frac{y^{2}}{8 N}} d y=\frac{\Gamma(2 N+3)}{\Gamma(N+2)}(4 N+3)^{-\frac{1}{2}}\left[(-1)^{N}(1-\cos x)+O\left(N^{-\frac{1}{2}}\right)\right], \quad N \rightarrow \infty .
$$

On the other hand, from [8],

$$
\int_{0}^{\infty} H_{2 N+1}(y) \mathrm{e}^{-\frac{y^{2}}{2}} d y=\frac{2(-1)^{N} \Gamma(2 N+2)}{\Gamma(N+1)}{ }_{2} F_{1}\left(-N, 1 ; \frac{3}{2} ; 2\right) .
$$

It follows from Lemma 2.2, that,

$$
\int_{0}^{\infty} H_{2 N+1}(y) \mathrm{e}^{-\frac{y^{2}}{2}} d y=\frac{2(-1)^{N} \Gamma(2 N+2)}{\Gamma(N+1)}\left[(-1)^{N} \sqrt{\frac{\pi}{8 N}}+O\left(N^{-1}\right)\right], \quad N \rightarrow \infty .
$$


By the Stirling's formula (2.1), we obtain

$$
\begin{aligned}
\varepsilon \varphi_{2 N+1}\left(\frac{x}{\sqrt{4 N}}\right) & =\left[2^{-1}(-1)^{N} \pi^{-\frac{1}{2}} N^{-\frac{3}{4}}(1-\cos x)+O\left(N^{-\frac{5}{4}}\right)\right]-\left[2^{-\frac{1}{2}} N^{-\frac{1}{4}}+O\left(N^{-\frac{3}{4}}\right)\right] \\
& =-2^{-\frac{1}{2}} N^{-\frac{1}{4}}+O\left(N^{-\frac{3}{4}}\right), \quad N \rightarrow \infty
\end{aligned}
$$

We are now in a position to compute $\operatorname{Tr} T$ and $\operatorname{Tr} T^{2}$ as $N \rightarrow \infty$, using Theorem 3.6 and Theorem 3.7. The estimates provided by Theorem 3.7 are instrumental in the large $N$ computations that follows.

In what follows, we replace $f(x)$ by $f(\sqrt{4 N} x)$, and note that

$$
f^{\prime}(\sqrt{4 N} x)=\frac{1}{\sqrt{4 N}} \frac{d}{d x} f(\sqrt{4 N} x)
$$

The $f^{\prime}$ that appears in the trace will be accordingly interpreted.

We first consider $\operatorname{Tr} T$, which reads,

$$
\operatorname{Tr} T=\operatorname{Tr} S_{N} f-\operatorname{Tr} \frac{1}{2} S_{N} \varepsilon f^{\prime}+\operatorname{Tr} \sqrt{N+\frac{1}{2}}\left(\varepsilon \varphi_{2 N+1} \otimes \varphi_{2 N} f\right)+\operatorname{Tr} \frac{1}{2} \sqrt{N+\frac{1}{2}}\left(\varepsilon \varphi_{2 N+1} \otimes \varepsilon \varphi_{2 N}\right) f^{\prime} .
$$

$\operatorname{Tr} T$ has four parts.

First of all, we find

$$
\begin{aligned}
\operatorname{Tr} S_{N} f & =\int_{-\infty}^{\infty} S_{N}(x, x) f(\sqrt{4 N} x) d x \\
& =\int_{-\infty}^{\infty} \frac{1}{\sqrt{4 N}} S_{N}\left(\frac{x}{\sqrt{4 N}}, \frac{x}{\sqrt{4 N}}\right) f(x) d x \\
& \rightarrow \frac{1}{\pi} \int_{-\infty}^{\infty} f(x) d x, \quad N \rightarrow \infty
\end{aligned}
$$

The second term reads,

$$
\begin{aligned}
\operatorname{Tr} \frac{1}{2} S_{N} \varepsilon f^{\prime} & =\frac{1}{2} \int_{-\infty}^{\infty} \int_{-\infty}^{\infty} S_{N}(x, y) \varepsilon(y, x) f^{\prime}(\sqrt{4 N} x) d x d y \\
& =\frac{1}{4} \int_{-\infty}^{\infty}\left[\int_{x}^{\infty} S_{N}(x, y) d y-\int_{-\infty}^{x} S_{N}(x, y) d y\right] f^{\prime}(\sqrt{4 N} x) d x
\end{aligned}
$$

To proceed further, let

$$
u=\sqrt{4 N} x, \quad v=\sqrt{4 N} y,
$$


it follows that,

$$
\begin{aligned}
\operatorname{Tr} \frac{1}{2} S_{N} \varepsilon f^{\prime} & =\frac{1}{4 \sqrt{4 N}} \int_{-\infty}^{\infty}\left[\int_{u}^{\infty} \frac{1}{\sqrt{4 N}} S_{N}\left(\frac{u}{\sqrt{4 N}}, \frac{v}{\sqrt{4 N}}\right) d v-\int_{-\infty}^{u} \frac{1}{\sqrt{4 N}} S_{N}\left(\frac{u}{\sqrt{4 N}}, \frac{v}{\sqrt{4 N}}\right) d v\right] f^{\prime}(u) d u \\
& \rightarrow \frac{1}{4 \sqrt{4 N} \pi} \int_{-\infty}^{\infty}\left[\int_{u}^{\infty} \frac{\sin (u-v)}{u-v} d v-\int_{-\infty}^{u} \frac{\sin (u-v)}{u-v} d v\right] f^{\prime}(u) d u, \quad N \rightarrow \infty
\end{aligned}
$$

Now let $t=u-v$, it follows that,

$$
\begin{aligned}
\operatorname{Tr} \frac{1}{2} S_{N} \varepsilon f^{\prime} & \rightarrow \frac{1}{4 \sqrt{4 N} \pi} \int_{-\infty}^{\infty}\left[\int_{-\infty}^{0} \frac{\sin t}{t} d t-\int_{0}^{\infty} \frac{\sin t}{t} d t\right] f^{\prime}(u) d u \\
& =\frac{1}{4 \sqrt{4 N} \pi} \int_{-\infty}^{\infty}\left(\frac{\pi}{2}-\frac{\pi}{2}\right) f^{\prime}(u) d u \\
& =0, \quad N \rightarrow \infty .
\end{aligned}
$$

For the third term, we have,

$$
\begin{aligned}
\operatorname{Tr} \sqrt{N+\frac{1}{2}}\left(\varepsilon \varphi_{2 N+1} \otimes \varphi_{2 N} f\right) & =\sqrt{N+\frac{1}{2}} \int_{-\infty}^{\infty} \varepsilon \varphi_{2 N+1}(x) \varphi_{2 N}(x) f(\sqrt{4 N} x) d x \\
& =\sqrt{N+\frac{1}{2}} \cdot \frac{1}{\sqrt{4 N}} \int_{-\infty}^{\infty} \varepsilon \varphi_{2 N+1}\left(\frac{x}{\sqrt{4 N}}\right) \varphi_{2 N}\left(\frac{x}{\sqrt{4 N}}\right) f(x) d x \\
& =-\frac{(-1)^{N}}{2 \sqrt{2 \pi N}} \int_{-\infty}^{\infty} \cos x f(x) d x+O\left(N^{-1}\right), \quad N \rightarrow \infty,
\end{aligned}
$$

where use has been made of Theorem 3.7 .

Finally to the fourth term, and take note of Theorem 3.7 .

$$
\begin{aligned}
\operatorname{Tr} \frac{1}{2} \sqrt{N+\frac{1}{2}}\left(\varepsilon \varphi_{2 N+1} \otimes \varepsilon \varphi_{2 N}\right) f^{\prime} & =\frac{1}{2} \sqrt{N+\frac{1}{2}} \int_{-\infty}^{\infty} \varepsilon \varphi_{2 N+1}(x) \varepsilon \varphi_{2 N}(x) f^{\prime}(\sqrt{4 N} x) d x \\
& =\frac{1}{2} \sqrt{N+\frac{1}{2}} \cdot \frac{1}{\sqrt{4 N}} \int_{-\infty}^{\infty} \varepsilon \varphi_{2 N+1}\left(\frac{x}{\sqrt{4 N}}\right) \varepsilon \varphi_{2 N}\left(\frac{x}{\sqrt{4 N}}\right) f^{\prime}(x) d x \\
& =O\left(N^{-1}\right), \quad N \rightarrow \infty
\end{aligned}
$$

Therefore, the large $N$ expansion of $\operatorname{Tr} T$, reads,

$$
\operatorname{Tr} T=\frac{1}{\pi} \int_{-\infty}^{\infty} f(x) d x-\frac{(-1)^{N}}{2 \sqrt{2 \pi N}} \int_{-\infty}^{\infty} \cos x f(x) d x+O\left(N^{-1}\right), \quad N \rightarrow \infty .
$$


Working out $\operatorname{Tr} T^{2}$, with $T$ given by, (3.14), there are 10 traces:

$$
\begin{aligned}
\operatorname{Tr} T^{2} & =\operatorname{Tr} S_{N} f S_{N} f-\operatorname{Tr} S_{N} f S_{N} \varepsilon f^{\prime}+\operatorname{Tr} \sqrt{4 N+2} S_{N} f\left(\varepsilon \varphi_{2 N+1} \otimes \varphi_{2 N} f\right) \\
& +\operatorname{Tr} \sqrt{N+\frac{1}{2}} S_{N} f\left(\varepsilon \varphi_{2 N+1} \otimes \varepsilon \varphi_{2 N}\right) f^{\prime}+\operatorname{Tr} \frac{1}{4} S_{N} \varepsilon f^{\prime} S_{N} \varepsilon f^{\prime} \\
& -\operatorname{Tr} \sqrt{N+\frac{1}{2}} S_{N} \varepsilon f^{\prime}\left(\varepsilon \varphi_{2 N+1} \otimes \varphi_{2 N} f\right)-\operatorname{Tr} \frac{1}{2} \sqrt{N+\frac{1}{2}} S_{N} \varepsilon f^{\prime}\left(\varepsilon \varphi_{2 N+1} \otimes \varepsilon \varphi_{2 N}\right) f^{\prime} \\
& +\operatorname{Tr}\left(N+\frac{1}{2}\right)\left(\varepsilon \varphi_{2 N+1} \otimes \varphi_{2 N} f\right)\left(\varepsilon \varphi_{2 N+1} \otimes \varphi_{2 N} f\right) \\
& +\operatorname{Tr}\left(N+\frac{1}{2}\right)\left(\varepsilon \varphi_{2 N+1} \otimes \varphi_{2 N} f\right)\left(\varepsilon \varphi_{2 N+1} \otimes \varepsilon \varphi_{2 N}\right) f^{\prime} \\
& +\operatorname{Tr} \frac{1}{4}\left(N+\frac{1}{2}\right)\left(\varepsilon \varphi_{2 N+1} \otimes \varepsilon \varphi_{2 N}\right) f^{\prime}\left(\varepsilon \varphi_{2 N+1} \otimes \varepsilon \varphi_{2 N}\right) f^{\prime}
\end{aligned}
$$

In the following, we calculate the trace on the right side of (3.16), term by term.

The first term:

$$
\begin{aligned}
\operatorname{Tr} S_{N} f S_{N} f & =\int_{-\infty}^{\infty} \int_{-\infty}^{\infty} S_{N}(x, y) f(\sqrt{4 N} y) S_{N}(y, x) f(\sqrt{4 N} x) d y d x \\
& =\int_{-\infty}^{\infty} \int_{-\infty}^{\infty}\left[\frac{1}{\sqrt{4 N}} S_{N}\left(\frac{x}{\sqrt{4 N}}, \frac{y}{\sqrt{4 N}}\right)\right]^{2} f(x) f(y) d x d y \\
& \rightarrow \frac{1}{\pi^{2}} \int_{-\infty}^{\infty} \int_{-\infty}^{\infty}\left[\frac{\sin (x-y)}{x-y}\right]^{2} f(x) f(y) d x d y, \quad N \rightarrow \infty
\end{aligned}
$$

The second term reads,

$$
\begin{aligned}
\operatorname{Tr} S_{N} f S_{N} \varepsilon f^{\prime} & =\int_{-\infty}^{\infty} \int_{-\infty}^{\infty} \int_{-\infty}^{\infty} S_{N}(x, y) f(\sqrt{4 N} y) S_{N}(y, z) \varepsilon(z, x) f^{\prime}(\sqrt{4 N} x) d x d y d z \\
& =\frac{1}{2} \int_{-\infty}^{\infty} \int_{-\infty}^{\infty} S_{N}(x, y)\left[\int_{x}^{\infty} S_{N}(y, z) d z-\int_{-\infty}^{x} S_{N}(y, z) d z\right] f^{\prime}(\sqrt{4 N} x) f(\sqrt{4 N} y) d x d y
\end{aligned}
$$

A change of variables,

$$
u=\sqrt{4 N} x, \quad v=\sqrt{4 N} y, \quad w=\sqrt{4 N} z,
$$

give

$$
\begin{aligned}
\operatorname{Tr} S_{N} f S_{N} \varepsilon f^{\prime}= & \frac{1}{2 \sqrt{4 N}} \int_{-\infty}^{\infty} \int_{-\infty}^{\infty} \frac{1}{\sqrt{4 N}} S_{N}\left(\frac{u}{\sqrt{4 N}}, \frac{v}{\sqrt{4 N}}\right) \\
& {\left[\int_{u}^{\infty} \frac{1}{\sqrt{4 N}} S_{N}\left(\frac{v}{\sqrt{4 N}}, \frac{w}{\sqrt{4 N}}\right) d w-\int_{-\infty}^{u} \frac{1}{\sqrt{4 N}} S_{N}\left(\frac{v}{\sqrt{4 N}}, \frac{w}{\sqrt{4 N}}\right) d w\right] f^{\prime}(u) f(v) d u d v } \\
\rightarrow & \frac{1}{2 \sqrt{4 N} \pi^{2}} \int_{-\infty}^{\infty} \int_{-\infty}^{\infty} \frac{\sin (u-v)}{u-v}\left[\int_{u}^{\infty} \frac{\sin (v-w)}{v-w} d w-\int_{-\infty}^{u} \frac{\sin (v-w)}{v-w} d w\right] \\
& f^{\prime}(u) f(v) d u d v, N \rightarrow \infty .
\end{aligned}
$$


To proceed further, let $t=v-w$, we see that,

$$
\begin{aligned}
& \int_{u}^{\infty} \frac{\sin (v-w)}{v-w} d w-\int_{-\infty}^{u} \frac{\sin (v-w)}{v-w} d w=\int_{-\infty}^{v-u} \frac{\sin t}{t} d t-\int_{v-u}^{\infty} \frac{\sin t}{t} d t=2 \int_{0}^{v-u} \frac{\sin t}{t} d t \\
= & 2 \operatorname{Si}(v-u),
\end{aligned}
$$

where $\operatorname{Si}(x)$ is the sine integral

$$
\operatorname{Si}(x):=\int_{0}^{x} \frac{\sin t}{t} d t
$$

Since $\operatorname{Si}(-x)=-\operatorname{Si}(x)$, it follows that

$$
\begin{aligned}
\operatorname{Tr} S_{N} f S_{N} \varepsilon f^{\prime} & \rightarrow \frac{1}{\sqrt{4 N} \pi^{2}} \int_{-\infty}^{\infty} \int_{-\infty}^{\infty} \frac{\sin (u-v)}{u-v} \operatorname{Si}(v-u) f^{\prime}(u) f(v) d u d v \\
& =-\frac{1}{2 \pi^{2} \sqrt{N}} \int_{-\infty}^{\infty} \int_{-\infty}^{\infty} \frac{\sin (u-v)}{u-v} \operatorname{Si}(u-v) f^{\prime}(u) f(v) d u d v, N \rightarrow \infty
\end{aligned}
$$

The third term:

$$
\begin{aligned}
& \operatorname{Tr} \sqrt{4 N+2} S_{N} f\left(\varepsilon \varphi_{2 N+1} \otimes \varphi_{2 N} f\right) \\
= & \sqrt{4 N+2} \int_{-\infty}^{\infty} \int_{-\infty}^{\infty} S_{N}(x, y) f(\sqrt{4 N} y) \varepsilon \varphi_{2 N+1}(y) \varphi_{2 N}(x) f(\sqrt{4 N} x) d x d y \\
= & \sqrt{4 N+2} \cdot \frac{1}{\sqrt{4 N}} \int_{-\infty}^{\infty} \int_{-\infty}^{\infty} \frac{1}{\sqrt{4 N}} S_{N}\left(\frac{x}{\sqrt{4 N}}, \frac{y}{\sqrt{4 N}}\right) f(y) \varepsilon \varphi_{2 N+1}\left(\frac{y}{\sqrt{4 N}}\right) \varphi_{2 N}\left(\frac{x}{\sqrt{4 N}}\right) f(x) d x d y \\
= & -\frac{(-1)^{N}}{\pi \sqrt{2 \pi N}} \int_{-\infty}^{\infty} \int_{-\infty}^{\infty} \frac{\sin (x-y)}{x-y} \cos x f(x) f(y) d x d y+O\left(N^{-1}\right), N \rightarrow \infty .
\end{aligned}
$$

The fourth term:

$$
\begin{aligned}
& \operatorname{Tr} \sqrt{N+\frac{1}{2}} S_{N} f\left(\varepsilon \varphi_{2 N+1} \otimes \varepsilon \varphi_{2 N}\right) f^{\prime} \\
= & \sqrt{N+\frac{1}{2}} \int_{-\infty}^{\infty} \int_{-\infty}^{\infty} S_{N}(x, y) f(\sqrt{4 N} y) \varepsilon \varphi_{2 N+1}(y) \varepsilon \varphi_{2 N}(x) f^{\prime}(\sqrt{4 N} x) d x d y \\
= & \sqrt{N+\frac{1}{2}} \cdot \frac{1}{\sqrt{4 N}} \int_{-\infty}^{\infty} \int_{-\infty}^{\infty} \frac{1}{\sqrt{4 N}} S_{N}\left(\frac{x}{\sqrt{4 N}}, \frac{y}{\sqrt{4 N}}\right) f(y) \varepsilon \varphi_{2 N+1}\left(\frac{y}{\sqrt{4 N}}\right) \varepsilon \varphi_{2 N}\left(\frac{x}{\sqrt{4 N}}\right) f^{\prime}(x) d x d y \\
= & O\left(N^{-1}\right), N \rightarrow \infty .
\end{aligned}
$$

The fifth term, with the change of variables,

$$
u=\sqrt{4 N} x, \quad v=\sqrt{4 N} y, \quad w=\sqrt{4 N} z, \quad \tau=\sqrt{4 N} t
$$


we see that,

$$
\begin{aligned}
& \operatorname{Tr} \frac{1}{4} S_{N} \varepsilon f^{\prime} S_{N} \varepsilon f^{\prime} \\
= & \frac{1}{64 N} \int_{-\infty}^{\infty} \int_{-\infty}^{\infty}\left[\int_{w}^{\infty} \frac{1}{\sqrt{4 N}} S_{N}\left(\frac{u}{\sqrt{4 N}}, \frac{v}{\sqrt{4 N}}\right) d v-\int_{-\infty}^{w} \frac{1}{\sqrt{4 N}} S_{N}\left(\frac{u}{\sqrt{4 N}}, \frac{v}{\sqrt{4 N}}\right) d v\right] \\
& {\left[\int_{u}^{\infty} \frac{1}{\sqrt{4 N}} S_{N}\left(\frac{w}{\sqrt{4 N}}, \frac{\tau}{\sqrt{4 N}}\right) d \tau-\int_{-\infty}^{u} \frac{1}{\sqrt{4 N}} S_{N}\left(\frac{w}{\sqrt{4 N}}, \frac{\tau}{\sqrt{4 N}}\right) d \tau\right] f^{\prime}(u) f^{\prime}(w) d u d w } \\
= & O\left(N^{-1}\right), N \rightarrow \infty .
\end{aligned}
$$

The sixth term,

$$
\begin{aligned}
& \operatorname{Tr} \sqrt{N+\frac{1}{2}} S_{N} \varepsilon f^{\prime}\left(\varepsilon \varphi_{2 N+1} \otimes \varphi_{2 N} f\right) \\
= & \frac{1}{8 N} \sqrt{N+\frac{1}{2}} \int_{-\infty}^{\infty} \int_{-\infty}^{\infty}\left[\int_{w}^{\infty} \frac{1}{\sqrt{4 N}} S_{N}\left(\frac{u}{\sqrt{4 N}}, \frac{v}{\sqrt{4 N}}\right) d v-\int_{-\infty}^{w} \frac{1}{\sqrt{4 N}} S_{N}\left(\frac{u}{\sqrt{4 N}}, \frac{v}{\sqrt{4 N}}\right) d v\right] \\
& \varphi_{2 N}\left(\frac{u}{\sqrt{4 N}}\right) \varepsilon \varphi_{2 N+1}\left(\frac{w}{\sqrt{4 N}}\right) f(u) f^{\prime}(w) d u d w \\
= & O\left(N^{-1}\right), N \rightarrow \infty .
\end{aligned}
$$

The seventh term, becomes,

$$
\begin{aligned}
& \operatorname{Tr} \frac{1}{2} \sqrt{N+\frac{1}{2}} S_{N} \varepsilon f^{\prime}\left(\varepsilon \varphi_{2 N+1} \otimes \varepsilon \varphi_{2 N}\right) f^{\prime} \\
= & \frac{1}{16 N} \sqrt{N+\frac{1}{2}} \int_{-\infty}^{\infty} \int_{-\infty}^{\infty}\left[\int_{w}^{\infty} \frac{1}{\sqrt{4 N}} S_{N}\left(\frac{u}{\sqrt{4 N}}, \frac{v}{\sqrt{4 N}}\right) d v-\int_{-\infty}^{w} \frac{1}{\sqrt{4 N}} S_{N}\left(\frac{u}{\sqrt{4 N}}, \frac{v}{\sqrt{4 N}}\right) d v\right] \\
& \varepsilon \varphi_{2 N}\left(\frac{u}{\sqrt{4 N}}\right) \varepsilon \varphi_{2 N+1}\left(\frac{w}{\sqrt{4 N}}\right) f^{\prime}(u) f^{\prime}(w) d u d w \\
= & O\left(N^{-\frac{3}{2}}\right), N \rightarrow \infty .
\end{aligned}
$$

The eighth term can be computed in a similar manner, and we have,

$$
\begin{aligned}
& \operatorname{Tr}\left(N+\frac{1}{2}\right)\left(\varepsilon \varphi_{2 N+1} \otimes \varphi_{2 N} f\right)\left(\varepsilon \varphi_{2 N+1} \otimes \varphi_{2 N} f\right) \\
= & \frac{N+\frac{1}{2}}{4 N} \int_{-\infty}^{\infty} \int_{-\infty}^{\infty} \varepsilon \varphi_{2 N+1}\left(\frac{u}{\sqrt{4 N}}\right) \varepsilon \varphi_{2 N+1}\left(\frac{v}{\sqrt{4 N}}\right) \varphi_{2 N}\left(\frac{u}{\sqrt{4 N}}\right) \varphi_{2 N}\left(\frac{v}{\sqrt{4 N}}\right) f(u) f(v) d u d v \\
= & O\left(N^{-1}\right), N \rightarrow \infty .
\end{aligned}
$$

Proceeding in a similar manner with the ninth term, we have,

$$
\begin{aligned}
& \operatorname{Tr}\left(N+\frac{1}{2}\right)\left(\varepsilon \varphi_{2 N+1} \otimes \varphi_{2 N} f\right)\left(\varepsilon \varphi_{2 N+1} \otimes \varepsilon \varphi_{2 N}\right) f^{\prime} \\
= & \frac{N+\frac{1}{2}}{4 N} \int_{-\infty}^{\infty} \int_{-\infty}^{\infty} \varepsilon \varphi_{2 N+1}\left(\frac{u}{\sqrt{4 N}}\right) \varepsilon \varphi_{2 N+1}\left(\frac{v}{\sqrt{4 N}}\right) \varepsilon \varphi_{2 N}\left(\frac{u}{\sqrt{4 N}}\right) \varphi_{2 N}\left(\frac{v}{\sqrt{4 N}}\right) f^{\prime}(u) f(v) d u d v \\
= & O\left(N^{-\frac{3}{2}}\right), N \rightarrow \infty .
\end{aligned}
$$


The tenth and last term in $\operatorname{Tr} T^{2}$, becomes

$$
\begin{aligned}
& \operatorname{Tr} \frac{1}{4}\left(N+\frac{1}{2}\right)\left(\varepsilon \varphi_{2 N+1} \otimes \varepsilon \varphi_{2 N}\right) f^{\prime}\left(\varepsilon \varphi_{2 N+1} \otimes \varepsilon \varphi_{2 N}\right) f^{\prime} \\
= & \frac{N+\frac{1}{2}}{16 N} \int_{-\infty}^{\infty} \int_{-\infty}^{\infty} \varepsilon \varphi_{2 N+1}\left(\frac{u}{\sqrt{4 N}}\right) \varepsilon \varphi_{2 N+1}\left(\frac{v}{\sqrt{4 N}}\right) \varepsilon \varphi_{2 N}\left(\frac{u}{\sqrt{4 N}}\right) \varepsilon \varphi_{2 N}\left(\frac{v}{\sqrt{4 N}}\right) f^{\prime}(u) f^{\prime}(v) d u d v \\
= & O\left(N^{-2}\right), N \rightarrow \infty .
\end{aligned}
$$

Hence, the large $N$ behavior of (3.16) , reads,

$$
\begin{aligned}
\operatorname{Tr} T^{2} & =\frac{1}{\pi^{2}} \int_{-\infty}^{\infty} \int_{-\infty}^{\infty}\left[\frac{\sin (x-y)}{x-y}\right]^{2} f(x) f(y) d x d y \\
& +\frac{1}{2 \pi^{2} \sqrt{N}} \int_{-\infty}^{\infty} \int_{-\infty}^{\infty} \frac{\sin (x-y)}{x-y} \operatorname{Si}(x-y) f^{\prime}(x) f(y) d x d y \\
& -\frac{(-1)^{N}}{\pi \sqrt{2 \pi N}} \int_{-\infty}^{\infty} \int_{-\infty}^{\infty} \frac{\sin (x-y)}{x-y} \cos x f(x) f(y) d x d y+O\left(N^{-1}\right), N \rightarrow \infty
\end{aligned}
$$

We are now in a position to compute the mean and variance of the (scaled) linear statistics $\sum_{j=1}^{N} F\left(\sqrt{4 N} x_{j}\right)$, which are obtained as the coefficients of $\lambda$ and $\lambda^{2}$, of $\log \operatorname{det}(I+T)$.

Since

$$
f(\sqrt{4 N} x) \approx-\lambda F(\sqrt{4 N} x)+\frac{\lambda^{2}}{2} F^{2}(\sqrt{4 N} x)
$$

we replace $f$ with $-\lambda F+\frac{\lambda^{2}}{2} F^{2}$ in the expression of $\operatorname{Tr} T$ and $\operatorname{Tr} T^{2}$. A minor rearrangement gives,

$$
\begin{aligned}
& \log \operatorname{det}(I+T) \\
= & -\lambda\left\{\frac{1}{\pi} \int_{-\infty}^{\infty} F(x) d x-\frac{(-1)^{N}}{2 \sqrt{2 \pi N}} \int_{-\infty}^{\infty} \cos x F(x) d x+O\left(N^{-1}\right)\right\} \\
+ & \frac{\lambda^{2}}{2}\left\{\frac{1}{\pi} \int_{-\infty}^{\infty} F^{2}(x) d x-\frac{1}{\pi^{2}} \int_{-\infty}^{\infty} \int_{-\infty}^{\infty}\left[\frac{\sin (x-y)}{x-y}\right]^{2} F(x) F(y) d x d y\right. \\
- & \frac{1}{2 \pi^{2} \sqrt{N}} \int_{-\infty}^{\infty} \int_{-\infty}^{\infty} \frac{\sin (x-y)}{x-y} \operatorname{Si}(x-y) F^{\prime}(x) F(y) d x d y \\
- & \left.\frac{(-1)^{N}}{2 \sqrt{2 \pi N}}\left[\int_{-\infty}^{\infty} \cos x F^{2}(x) d x-\frac{2}{\pi} \int_{-\infty}^{\infty} \int_{-\infty}^{\infty} \frac{\sin (x-y)}{x-y} \cos x F(x) F(y) d x d y\right]+O\left(N^{-1}\right)\right\}, N \rightarrow \infty
\end{aligned}
$$

Denote by $\mu_{N}^{(G S E)}$ and $\mathcal{V}_{N}^{(G S E)}$ the mean and variance of the linear statistics $\sum_{j=1}^{N} F\left(\sqrt{4 N} x_{j}\right)$, then we have obtained, the large $N$ corrections of these quantities.

Theorem 3.8. As $N \rightarrow \infty$,

$$
\mu_{N}^{(G S E)}=\frac{1}{2} \mu_{N}^{(G U E)}-\frac{(-1)^{N}}{4 \sqrt{2 \pi N}} \int_{-\infty}^{\infty} \cos x F(x) d x+O\left(N^{-1}\right)
$$




$$
\begin{aligned}
\mathcal{V}_{N}^{(G S E)} & =\frac{1}{2} \mathcal{V}_{N}^{(G U E)}-\frac{1}{4 \pi^{2} \sqrt{N}} \int_{-\infty}^{\infty} \int_{-\infty}^{\infty} \frac{\sin (x-y)}{x-y} \operatorname{Si}(x-y) F^{\prime}(x) F(y) d x d y \\
& -\frac{(-1)^{N}}{4 \sqrt{2 \pi N}}\left[\int_{-\infty}^{\infty} \cos x F^{2}(x) d x-\frac{2}{\pi} \int_{-\infty}^{\infty} \int_{-\infty}^{\infty} \frac{\sin (x-y)}{x-y} \cos x F(x) F(y) d x d y\right]+O\left(N^{-1}\right)
\end{aligned}
$$

where $\mu_{N}^{(G U E)}$ and $\mathcal{V}_{N}^{(G U E)}$ for $N \rightarrow \infty$ are given in (1.2) and (1.3) respectively.

\subsection{LSE}

We study the case with the Laguerre background, namely, the weight, $w(x)=x^{\alpha} \mathrm{e}^{-x}, \quad \alpha>0, \quad x \in \mathbb{R}^{+}$. The idea is to choose special $\psi_{j}$ so that $M^{(4)}$ takes on the simplest possible form. To this end, let

$$
\begin{aligned}
& \psi_{2 j+1}(x):=\frac{1}{\sqrt{2}} \varphi_{2 j+1}^{(\alpha-1)}(x), \quad j=0,1,2, \ldots, \\
& \psi_{2 j}(x):=-\frac{1}{\sqrt{2}} \varepsilon \widetilde{\varphi}_{2 j+1}^{(\alpha-1)}(x), \quad j=0,1,2, \ldots,
\end{aligned}
$$

where $\varphi_{j}^{(\alpha-1)}(x)$ and $\widetilde{\varphi}_{j}^{(\alpha-1)}(x)$ are given by

$$
\begin{gathered}
\varphi_{j}^{(\alpha-1)}(x)=\frac{L_{j}^{(\alpha-1)}(x)}{c_{j}^{(\alpha-1)}} x^{\frac{\alpha}{2}} \mathrm{e}^{-\frac{x}{2}}, \quad j=0,1,2, \ldots, \\
\widetilde{\varphi}_{j}^{(\alpha-1)}(x)=\frac{L_{j}^{(\alpha-1)}(x)}{c_{j}^{(\alpha-1)}} x^{\frac{\alpha}{2}-1} \mathrm{e}^{-\frac{x}{2}}, \quad j=0,1,2, \ldots
\end{gathered}
$$

Here $L_{j}^{(\alpha)}(x), j=0,1,2, \ldots$, are the Laguerre polynomials, with the orthogonality condition,

$$
\int_{0}^{\infty} L_{j}^{(\alpha)}(x) L_{k}^{(\alpha)}(x) x^{\alpha} \mathrm{e}^{-x} d x=\left(c_{j}^{(\alpha)}\right)^{2} \delta_{j k}, \quad c_{j}^{(\alpha)}=\sqrt{\frac{\Gamma(j+\alpha+1)}{\Gamma(j+1)}} .
$$

It is easy to see that

$$
\int_{0}^{\infty} \varphi_{j}^{(\alpha-1)}(x) \widetilde{\varphi}_{k}^{(\alpha-1)}(x) d x=\delta_{j k}, \quad j, k=0,1,2, \ldots
$$

We now prove that (3.17) and (3.18) satisfy (3.1), i.e., $\psi_{j}(x)=\pi_{j}(x) x^{\frac{\alpha}{2}} \mathrm{e}^{-\frac{x}{2}}, j=0,1,2, \ldots$, where $\pi_{j}(x)$ is a polynomial of degree $j$.

Theorem 3.9. $\psi_{j}(x)=\pi_{j}(x) x^{\frac{\alpha}{2}} \mathrm{e}^{-\frac{x}{2}}, j=0,1,2, \ldots$, where $\pi_{j}(x)$ is a polynomial of degree $j$. 
Proof. We prove this by considering two cases, $j$ odd, and $j$ even. If $j=2 n+1$, then by (3.17),

$$
\pi_{2 n+1}(x)=\frac{1}{\sqrt{2} c_{2 n+1}^{(\alpha-1)}} L_{2 n+1}^{(\alpha-1)}(x)
$$

Let $j=2 n$, then

$$
\psi_{2 n}(x)=-\frac{1}{\sqrt{2}} \varepsilon \widetilde{\varphi}_{2 n+1}(x)=-\frac{1}{\sqrt{2} c_{2 n+1}^{(\alpha-1)}} \int_{0}^{x} L_{2 n+1}^{(\alpha-1)}(y) y^{\frac{\alpha}{2}-1} \mathrm{e}^{-\frac{y}{2}} d y
$$

and we have used the fact $\int_{0}^{\infty} L_{2 n+1}^{(\alpha-1)}(y) y^{\frac{\alpha}{2}-1} \mathrm{e}^{-\frac{y}{2}} d y=0, n=0,1,2, \ldots$

Let us rewrite the above as

$$
\int_{0}^{x} L_{2 n+1}^{(\alpha-1)}(y) y^{\frac{\alpha}{2}-1} \mathrm{e}^{-\frac{y}{2}} d y=\widehat{\pi}_{2 n}(x) x^{\frac{\alpha}{2}} \mathrm{e}^{-\frac{x}{2}}, \quad n=0,1,2, \ldots
$$

where

$$
\widehat{\pi}_{2 n}(x):=-\sqrt{2} c_{2 n+1}^{(\alpha-1)} \pi_{2 n}(x)
$$

Take a derivative on both sides,

$$
L_{2 n+1}^{(\alpha-1)}(x) x^{\frac{\alpha}{2}-1} \mathrm{e}^{-\frac{x}{2}}=\left(x \widehat{\pi}_{2 n}^{\prime}(x)+\frac{\alpha}{2} \widehat{\pi}_{2 n}(x)-\frac{1}{2} x \widehat{\pi}_{2 n}(x)\right) x^{\frac{\alpha}{2}-1} \mathrm{e}^{-\frac{x}{2}}
$$

which becomes,

$$
L_{2 n+1}^{(\alpha-1)}(x)=x \widehat{\pi}_{2 n}^{\prime}(x)+\frac{\alpha}{2} \widehat{\pi}_{2 n}(x)-\frac{1}{2} x \widehat{\pi}_{2 n}(x) .
$$

Note that equation (3.20) is equivalent to (3.21). Now we seek to solve (3.21).

Suppose

$$
\widehat{\pi}_{2 n}(x):=a_{0}(n)+a_{1}(n) x+a_{2}(n) x^{2}+\cdots+a_{2 n-1}(n) x^{2 n-1}+a_{2 n}(n) x^{2 n},
$$

we see that the right side of (3.21) is equal to

$$
\begin{aligned}
& x \widehat{\pi}_{2 n}^{\prime}(x)+\frac{\alpha}{2} \widehat{\pi}_{2 n}(x)-\frac{1}{2} x \widehat{\pi}_{2 n}(x) \\
= & \frac{\alpha}{2} a_{0}(n)+\left[\left(1+\frac{\alpha}{2}\right) a_{1}(n)-\frac{1}{2} a_{0}(n)\right] x+\left[\left(2+\frac{\alpha}{2}\right) a_{2}(n)-\frac{1}{2} a_{1}(n)\right] x^{2}+\cdots \\
+ & {\left[\left(2 n-1+\frac{\alpha}{2}\right) a_{2 n-1}(n)-\frac{1}{2} a_{2 n-2}(n)\right] x^{2 n-1}+\left[\left(2 n+\frac{\alpha}{2}\right) a_{2 n}(n)-\frac{1}{2} a_{2 n-1}(n)\right] x^{2 n} } \\
- & \frac{1}{2} a_{2 n}(n) x^{2 n+1} .
\end{aligned}
$$


On the other hand, the left side of (3.21) is equal to

$$
\begin{aligned}
L_{2 n+1}^{(\alpha-1)}(x) & =\left(\begin{array}{c}
2 n+\alpha \\
2 n+1
\end{array}\right)-\left(\begin{array}{c}
2 n+\alpha \\
2 n
\end{array}\right) x+\frac{1}{2 !}\left(\begin{array}{c}
2 n+\alpha \\
2 n-1
\end{array}\right) x^{2}-\frac{1}{3 !}\left(\begin{array}{c}
2 n+\alpha \\
2 n-2
\end{array}\right) x^{3} \\
& +\cdots+\frac{1}{(2 n) !}\left(\begin{array}{c}
2 n+\alpha \\
1
\end{array}\right) x^{2 n}-\frac{1}{(2 n+1) !}\left(\begin{array}{c}
2 n+\alpha \\
0
\end{array}\right) x^{2 n+1}
\end{aligned}
$$

Compare the coefficients of (3.22) and (3.23), we have the equations

$$
\left\{\begin{array}{l}
\frac{\alpha}{2} a_{0}(n)=\left(\begin{array}{c}
2 n+\alpha \\
2 n+1
\end{array}\right) \\
\left(1+\frac{\alpha}{2}\right) a_{1}(n)-\frac{1}{2} a_{0}(n)=-\left(\begin{array}{c}
2 n+\alpha \\
2 n
\end{array}\right) \\
\left(2+\frac{\alpha}{2}\right) a_{2}(n)-\frac{1}{2} a_{1}(n)=\frac{1}{2 !}\left(\begin{array}{c}
2 n+\alpha \\
2 n-1
\end{array}\right) \\
\left(3+\frac{\alpha}{2}\right) a_{3}(n)-\frac{1}{2} a_{2}(n)=-\frac{1}{3 !}\left(\begin{array}{c}
2 n+\alpha \\
2 n-2
\end{array}\right) \\
\vdots \\
\left(2 n-1+\frac{\alpha}{2}\right) a_{2 n-1}(n)-\frac{1}{2} a_{2 n-2}(n)=-\frac{1}{(2 n-1) !}\left(\begin{array}{c}
2 n+\alpha \\
2
\end{array}\right) \\
\left(2 n+\frac{\alpha}{2}\right) a_{2 n}(n)-\frac{1}{2} a_{2 n-1}(n)=-\frac{1}{(2 n) !}\left(\begin{array}{c}
2 n+\alpha \\
1
\end{array}\right) \\
-\frac{1}{2} a_{2 n}(n)=-\frac{1}{(2 n+1) !}\left(\begin{array}{c}
2 n+\alpha \\
0
\end{array}\right) .
\end{array}\right.
$$

By solving the first $2 n+1$ equations in (3.24), we find,

$$
\left\{\begin{array}{l}
\frac{1}{2} a_{0}(n)=\frac{1}{\alpha}\left(\begin{array}{c}
2 n+\alpha \\
2 n+1
\end{array}\right) \\
\frac{1}{2} a_{1}(n)=\frac{1}{\alpha(\alpha+2)}\left(\begin{array}{c}
2 n+\alpha \\
2 n+1
\end{array}\right)-\frac{1}{\alpha+2}\left(\begin{array}{c}
2 n+\alpha \\
2 n
\end{array}\right) \\
\frac{1}{2} a_{2}(n)=\frac{1}{\alpha(\alpha+2)(\alpha+4)}\left(\begin{array}{c}
2 n+\alpha \\
2 n+1
\end{array}\right)-\frac{1}{(\alpha+2)(\alpha+4)}\left(\begin{array}{c}
2 n+\alpha \\
2 n
\end{array}\right)+\frac{1}{2 !} \frac{1}{\alpha+4}\left(\begin{array}{c}
2 n+\alpha \\
2 n-1
\end{array}\right) \\
\frac{1}{2} a_{3}(n)=\frac{1}{\alpha(\alpha+2)(\alpha+4)(\alpha+6)}\left(\begin{array}{c}
2 n+\alpha \\
2 n+1
\end{array}\right)-\frac{1}{(\alpha+2)(\alpha+4)(\alpha+6)}\left(\begin{array}{c}
2 n+\alpha \\
2 n
\end{array}\right)+\frac{1}{2 !} \frac{1}{(\alpha+4)(\alpha+6)}\left(\begin{array}{c}
2 n+\alpha \\
2 n-1
\end{array}\right)-\frac{1}{3 !} \frac{1}{\alpha+6}\left(\begin{array}{c}
2 n+\alpha \\
2 n-2
\end{array}\right) \\
\frac{1}{2} a_{2 n}(n)=\frac{1}{\alpha(\alpha+2)(\alpha+4) \cdots(\alpha+4 n)}\left(\begin{array}{c}
2 n+\alpha \\
2 n+1
\end{array}\right)-\frac{1}{(\alpha+2)(\alpha+4) \cdots(\alpha+4 n)}\left(\begin{array}{c}
2 n+\alpha \\
2 n
\end{array}\right)+\frac{1}{2 !} \frac{1}{(\alpha+4)(\alpha+6) \cdots(\alpha+4 n)}\left(\begin{array}{c}
2 n+\alpha \\
2 n-1
\end{array}\right) \\
-\frac{1}{3 !} \frac{1}{(\alpha+6) \cdots(\alpha+4 n)}\left(\begin{array}{c}
2 n+\alpha \\
2 n-2
\end{array}\right)+\cdots-\frac{1}{(2 n-1) !} \frac{1}{(\alpha+4 n-2)(\alpha+4 n)}\left(\begin{array}{c}
2 n+\alpha \\
2
\end{array}\right)+\frac{1}{(2 n) !} \frac{1}{\alpha+4 n}\left(\begin{array}{c}
2 n+\alpha \\
1
\end{array}\right) .
\end{array}\right.
$$

The last equation of (3.24), simplifies to,

$$
a_{2 n}(n)=\frac{2}{(2 n+1) !}
$$

By Lemma 2.3, we see that linear system (3.24) is solvable. Hence $\widehat{\pi}_{2 n}(x)$ is a polynomial of degree $2 n$. The proof is complete. 
With (3.17) and (3.18) , we compute $M^{(4)}:=\left(\int_{0}^{\infty}\left(\psi_{j}(x) \psi_{k}^{\prime}(x)-\psi_{j}^{\prime}(x) \psi_{k}(x)\right) d x\right)_{j, k=0}^{2 N-1}$, resulting in the following theorem.

\section{Theorem 3.10.}

$$
M^{(4)}=\left(\begin{array}{ccccccc}
0 & 1 & 0 & 0 & \cdots & 0 & 0 \\
-1 & 0 & 0 & 0 & \cdots & 0 & 0 \\
0 & 0 & 0 & 1 & \cdots & 0 & 0 \\
0 & 0 & -1 & 0 & \cdots & 0 & 0 \\
\vdots & \vdots & \vdots & \vdots & & \vdots & \vdots \\
0 & 0 & 0 & 0 & \cdots & 0 & 1 \\
0 & 0 & 0 & 0 & \cdots & -1 & 0
\end{array}\right)_{2 N \times 2 N}
$$

Proof. Let $m_{j k}$ be the $(j, k)$-entry of $M^{(4)}$, i.e.,

$$
m_{j k}:=\int_{0}^{\infty}\left(\psi_{j}(x) \psi_{k}^{\prime}(x)-\psi_{j}^{\prime}(x) \psi_{k}(x)\right) d x, \quad j, k=0,1, \ldots, 2 N-1
$$

We compute $m_{j, k}$ by considering four cases: $(j, k)=$ (even, odd), (odd, even), (even, even), (odd, odd).

For the (even, odd) case,

$$
\begin{aligned}
m_{2 j, 2 k+1} & =\int_{0}^{\infty}\left(\psi_{2 j}(x) \psi_{2 k+1}^{\prime}(x)-\psi_{2 j}^{\prime}(x) \psi_{2 k+1}(x)\right) d x \\
& =\int_{0}^{\infty} \psi_{2 j}(x) \psi_{2 k+1}^{\prime}(x) d x-\int_{0}^{\infty} \psi_{2 j}^{\prime}(x) \psi_{2 k+1}(x) d x \\
& =\left(\left.\psi_{2 j}(x) \psi_{2 k+1}(x)\right|_{0} ^{\infty}-\int_{0}^{\infty} \psi_{2 j}^{\prime}(x) \psi_{2 k+1}(x) d x\right)-\int_{0}^{\infty} \psi_{2 j}^{\prime}(x) \psi_{2 k+1}(x) d x \\
& =-2 \int_{0}^{\infty} \psi_{2 j}^{\prime}(x) \psi_{2 k+1}(x) d x \\
& =-2 \int_{0}^{\infty}\left(-\frac{1}{\sqrt{2}} \widetilde{\varphi}_{2 j+1}^{(\alpha-1)}(x)\right)\left(\frac{1}{\sqrt{2}} \varphi_{2 k+1}^{(\alpha-1)}(x)\right) d x \\
& =\int_{0}^{\infty} \widetilde{\varphi}_{2 j+1}^{(\alpha-1)}(x) \varphi_{2 k+1}^{(\alpha-1)}(x) d x \\
& =\delta_{j k} .
\end{aligned}
$$

For the (odd, even) case, since $M^{(4)}$ is antisymmetric, we have

$$
\begin{aligned}
m_{2 j+1,2 k} & =-m_{2 k, 2 j+1} \\
& =-\delta_{j, k} .
\end{aligned}
$$


For the (even, even) case and $j>k$,

$$
\begin{aligned}
m_{2 j, 2 k} & =\int_{0}^{\infty}\left(\psi_{2 j}(x) \psi_{2 k}^{\prime}(x)-\psi_{2 j}^{\prime}(x) \psi_{2 k}(x)\right) d x \\
& =\int_{0}^{\infty} \psi_{2 j}(x) \psi_{2 k}^{\prime}(x) d x-\int_{0}^{\infty} \psi_{2 j}^{\prime}(x) \psi_{2 k}(x) d x \\
& =\left.\psi_{2 j}(x) \psi_{2 k}(x)\right|_{0} ^{\infty}-2 \int_{0}^{\infty} \psi_{2 j}^{\prime}(x) \psi_{2 k}(x) d x \\
& =-2 \int_{0}^{\infty} \psi_{2 j}^{\prime}(x) \psi_{2 k}(x) d x \\
& =-2 \int_{0}^{\infty}\left(-\frac{1}{\sqrt{2} c_{2 j+1}^{(\alpha-1)}} L_{2 j+1}^{(\alpha-1)}(x) x^{\frac{\alpha}{2}-1} \mathrm{e}^{-\frac{x}{2}}\right)\left(\pi_{2 k}(x) x^{\frac{\alpha}{2}} \mathrm{e}^{-\frac{x}{2}}\right) d x \\
& =\frac{\sqrt{2}}{c_{2 j+1}^{(\alpha-1)}} \int_{0}^{\infty} L_{2 j+1}^{(\alpha-1)}(x) \pi_{2 k}(x) x^{\alpha-1} \mathrm{e}^{-x} d x \\
& =0,
\end{aligned}
$$

since $\pi_{2 k}(x)$ is a polynomial of degree $2 k$ which is less than $2 j+1$.

For the (odd, odd) case and $j>k$,

$$
\begin{aligned}
m_{2 j+1,2 k+1} & =\int_{0}^{\infty}\left(\psi_{2 j+1}(x) \psi_{2 k+1}^{\prime}(x)-\psi_{2 j+1}^{\prime}(x) \psi_{2 k+1}(x)\right) d x \\
& =\int_{0}^{\infty} \psi_{2 j+1}(x) \psi_{2 k+1}^{\prime}(x) d x-\int_{0}^{\infty} \psi_{2 j+1}^{\prime}(x) \psi_{2 k+1}(x) d x \\
& =\int_{0}^{\infty} \psi_{2 j+1}(x) \psi_{2 k+1}^{\prime}(x) d x-\left(\left.\psi_{2 j+1}(x) \psi_{2 k+1}(x)\right|_{0} ^{\infty}-\int_{0}^{\infty} \psi_{2 j+1}(x) \psi_{2 k+1}^{\prime}(x) d x\right) \\
& =2 \int_{0}^{\infty} \psi_{2 j+1}(x) \psi_{2 k+1}^{\prime}(x) d x \\
& =\frac{1}{c_{2 j+1}^{(\alpha-1)} c_{2 k+1}^{(\alpha-1)}} \int_{0}^{\infty} L_{2 j+1}^{(\alpha-1)}(x) x^{\frac{\alpha}{2}} \mathrm{e}^{-\frac{x}{2}}\left(x\left(L_{2 k+1}^{(\alpha-1)}(x)\right)^{\prime}+\frac{\alpha}{2} L_{2 k+1}^{(\alpha-1)}(x)-\frac{1}{2} x L_{2 k+1}^{(\alpha-1)}(x)\right) x^{\frac{\alpha}{2}-1} \mathrm{e}^{-\frac{x}{2}} d x \\
& =\frac{1}{c_{2 j+1}^{(\alpha-1)} c_{2 k+1}^{(\alpha-1)}} \int_{0}^{\infty} L_{2 j+1}^{(\alpha-1)}(x)\left(x\left(L_{2 k+1}^{(\alpha-1)}(x)\right)^{\prime}+\frac{\alpha}{2} L_{2 k+1}^{(\alpha-1)}(x)-\frac{1}{2} x L_{2 k+1}^{(\alpha-1)}(x)\right) x^{\alpha-1} \mathrm{e}^{-x} d x \\
& =0,
\end{aligned}
$$

since $x\left(L_{2 k+1}^{(\alpha-1)}(x)\right)^{\prime}+\frac{\alpha}{2} L_{2 k+1}^{(\alpha-1)}(x)-\frac{1}{2} x L_{2 k+1}^{(\alpha-1)}(x)$ is a polynomial of degree $2 k+2$ which is less than $2 j+1$.

If $j<k$, due to the fact that $M^{(4)}$ is antisymmetric,

$$
\begin{aligned}
m_{2 j, 2 k} & =-m_{2 k, 2 j} \\
& =0
\end{aligned}
$$




$$
\begin{aligned}
m_{2 j+1,2 k+1} & =-m_{2 k+1,2 j+1} \\
& =0 .
\end{aligned}
$$

Thus

$$
m_{2 j, 2 k}=m_{2 j+1,2 k+1}=0, \quad j, k=0,1, \ldots, N-1 .
$$

This is just the desired form of $M^{(4)}$.

It's clear that $\left(M^{(4)}\right)^{-1}=-M^{(4)}$, so $\mu_{2 j, 2 j+1}=-1, \mu_{2 j+1,2 j}=1$, and $\mu_{j k}=0$ for other cases. The rest of this subsection is devoted to the determination of $K_{N, 4}^{(2,2)}(x, y)$,

$$
\begin{aligned}
K_{N, 4}^{(2,2)}(x, y) & =-\sum_{j, k=0}^{2 N-1} \psi_{j}(x) \mu_{j k} \psi_{k}^{\prime}(y) \\
& =\sum_{j=0}^{N-1} \psi_{2 j}(x) \psi_{2 j+1}^{\prime}(y)-\sum_{j=0}^{N-1} \psi_{2 j+1}(x) \psi_{2 j}^{\prime}(y) \\
& =\frac{1}{2} \sum_{j=0}^{N-1} \varphi_{2 j+1}^{(\alpha-1)}(x) \widetilde{\varphi}_{2 j+1}^{(\alpha-1)}(y)-\frac{1}{2} \sum_{j=0}^{N-1} \varepsilon \widetilde{\varphi}_{2 j+1}^{(\alpha-1)}(x)\left[\varphi_{2 j+1}^{(\alpha-1)}(y)\right]^{\prime} .
\end{aligned}
$$

From (3.19), we see that,

$$
\left[\varphi_{j}^{(\alpha-1)}(x)\right]^{\prime}=\frac{1}{c_{j}^{(\alpha-1)}} x^{\frac{\alpha}{2}-1} \mathrm{e}^{-\frac{x}{2}}\left[x\left(L_{j}^{(\alpha-1)}(x)\right)^{\prime}+\frac{\alpha-x}{2} L_{j}^{(\alpha-1)}(x)\right] .
$$

Recall that the Laguerre polynomials $L_{j}^{(\alpha-1)}(x)$, satisfy the differentiation formulas [8],

$$
\begin{gathered}
x\left(L_{j}^{(\alpha-1)}(x)\right)^{\prime}=j L_{j}^{(\alpha-1)}(x)-(j+\alpha-1) L_{j-1}^{(\alpha-1)}(x), \quad j=0,1,2, \ldots, \\
x\left(L_{j}^{(\alpha-1)}(x)\right)^{\prime}=(j+1) L_{j+1}^{(\alpha-1)}(x)+(x-j-\alpha) L_{j}^{(\alpha-1)}(x), \quad j=0,1,2, \ldots .
\end{gathered}
$$

Summing (3.26) and (3.27), and divide by 2, gives,

$$
x\left(L_{j}^{(\alpha-1)}(x)\right)^{\prime}=\frac{j+1}{2} L_{j+1}^{(\alpha-1)}(x)-\frac{j+\alpha-1}{2} L_{j-1}^{(\alpha-1)}(x)+\frac{x-\alpha}{2} L_{j}^{(\alpha-1)}(x), \quad j=0,1,2, \ldots,
$$

or

$$
x\left(L_{j}^{(\alpha-1)}(x)\right)^{\prime}+\frac{\alpha-x}{2} L_{j}^{(\alpha-1)}(x)=\frac{j+1}{2} L_{j+1}^{(\alpha-1)}(x)-\frac{j+\alpha-1}{2} L_{j-1}^{(\alpha-1)}(x), \quad j=0,1,2, \ldots
$$

Hence (3.25) becomes,

$$
\begin{aligned}
{\left[\varphi_{j}^{(\alpha-1)}(x)\right]^{\prime} } & =\frac{1}{c_{j}^{(\alpha-1)}} x^{\frac{\alpha}{2}-1} \mathrm{e}^{-\frac{x}{2}}\left[\frac{j+1}{2} L_{j+1}^{(\alpha-1)}(x)-\frac{j+\alpha-1}{2} L_{j-1}^{(\alpha-1)}(x)\right] \\
& =\frac{1}{2} \sqrt{(j+1)(j+\alpha)} \widetilde{\varphi}_{j+1}^{(\alpha-1)}(x)-\frac{1}{2} \sqrt{j(j+\alpha-1)} \widetilde{\varphi}_{j-1}^{(\alpha-1)}(x) .
\end{aligned}
$$


We see that $\left[\varphi_{j}^{(\alpha-1)}\right]^{\prime}$ is a linear combination of $\widetilde{\varphi}_{j+1}^{(\alpha-1)}$ and $\widetilde{\varphi}_{j-1}^{(\alpha-1)}$, just like the GSE case studied in the last section. Replacing $j$ by $2 j+1$ in (3.28), to find,

$$
\left[\varphi_{2 j+1}^{(\alpha-1)}(y)\right]^{\prime}=\sqrt{(j+1)\left(j+\frac{\alpha+1}{2}\right)} \widetilde{\varphi}_{2 j+2}^{(\alpha-1)}(y)-\sqrt{\left(j+\frac{1}{2}\right)\left(j+\frac{\alpha}{2}\right)} \widetilde{\varphi}_{2 j}^{(\alpha-1)}(y) .
$$

A straightforward computation shows that,

$$
\begin{aligned}
& \sum_{j=0}^{N-1} \varepsilon \widetilde{\varphi}_{2 j+1}^{(\alpha-1)}(x)\left[\varphi_{2 j+1}^{(\alpha-1)}(y)\right]^{\prime} \\
= & \sum_{j=0}^{N-1} \varepsilon \widetilde{\varphi}_{2 j+1}^{(\alpha-1)}(x) \sqrt{(j+1)\left(j+\frac{\alpha+1}{2}\right)} \widetilde{\varphi}_{2 j+2}^{(\alpha-1)}(y)-\sum_{j=0}^{N-1} \varepsilon \widetilde{\varphi}_{2 j+1}^{(\alpha-1)}(x) \sqrt{\left(j+\frac{1}{2}\right)\left(j+\frac{\alpha}{2}\right)} \widetilde{\varphi}_{2 j}^{(\alpha-1)}(y) \\
= & \sum_{j=0}^{N} \sqrt{j\left(j+\frac{\alpha-1}{2}\right)} \varepsilon \widetilde{\varphi}_{2 j-1}^{(\alpha-1)}(x) \widetilde{\varphi}_{2 j}^{(\alpha-1)}(y)-\sum_{j=0}^{N} \sqrt{\left(j+\frac{1}{2}\right)\left(j+\frac{\alpha}{2}\right) \varepsilon \widetilde{\varphi}_{2 j+1}^{(\alpha-1)}(x) \widetilde{\varphi}_{2 j}^{(\alpha-1)}(y)} \\
+ & \sqrt{\left(N+\frac{1}{2}\right)\left(N+\frac{\alpha}{2}\right)} \varepsilon \widetilde{\varphi}_{2 N+1}^{(\alpha-1)}(x) \widetilde{\varphi}_{2 N}^{(\alpha-1)}(y) \\
= & \sum_{j=0}^{N}\left[\sqrt{j\left(j+\frac{\alpha-1}{2}\right)} \varepsilon \widetilde{\varphi}_{2 j-1}^{(\alpha-1)}(x)-\sqrt{\left(j+\frac{1}{2}\right)\left(j+\frac{\alpha}{2}\right)} \varepsilon \widetilde{\varphi}_{2 j+1}^{(\alpha-1)}(x)\right] \widetilde{\varphi}_{2 j}^{(\alpha-1)}(y) \\
+ & \sqrt{\left(N+\frac{1}{2}\right)\left(N+\frac{\alpha}{2}\right)} \varepsilon \widetilde{\varphi}_{2 N+1}^{(\alpha-1)}(x) \widetilde{\varphi}_{2 N}^{(\alpha-1)}(y) .
\end{aligned}
$$

From (3.28) and Theorem 2.7,

$$
\begin{aligned}
\varphi_{2 j}^{(\alpha-1)}(x) & =\varepsilon D \varphi_{2 j}^{(\alpha-1)}(x) \\
& =\varepsilon\left[\varphi_{2 j}^{(\alpha-1)}(x)\right]^{\prime} \\
& =\varepsilon\left[\frac{1}{2} \sqrt{(2 j+1)(2 j+\alpha)} \widetilde{\varphi}_{2 j+1}^{(\alpha-1)}(x)-\frac{1}{2} \sqrt{2 j(2 j+\alpha-1)} \widetilde{\varphi}_{2 j-1}^{(\alpha-1)}(x)\right] \\
& =\sqrt{\left(j+\frac{1}{2}\right)\left(j+\frac{\alpha}{2}\right)} \varepsilon \widetilde{\varphi}_{2 j+1}^{(\alpha-1)}(x)-\sqrt{j\left(j+\frac{\alpha-1}{2}\right)} \varepsilon \widetilde{\varphi}_{2 j-1}^{(\alpha-1)}(x) .
\end{aligned}
$$

Hence, the sum, $\sum_{j=0}^{N-1} \varepsilon \widetilde{\varphi}_{2 j+1}^{(\alpha-1)}(x)\left[\varphi_{2 j+1}^{(\alpha-1)}(y)\right]^{\prime}$ simplifies immediately, and leads to,

$$
\sum_{j=0}^{N-1} \varepsilon \widetilde{\varphi}_{2 j+1}^{(\alpha-1)}(x)\left[\varphi_{2 j+1}^{(\alpha-1)}(y)\right]^{\prime}=-\sum_{j=0}^{N} \varphi_{2 j}^{(\alpha-1)}(x) \widetilde{\varphi}_{2 j}^{(\alpha-1)}(y)+\sqrt{\left(N+\frac{1}{2}\right)\left(N+\frac{\alpha}{2}\right)} \varepsilon \widetilde{\varphi}_{2 N+1}^{(\alpha-1)}(x) \widetilde{\varphi}_{2 N}^{(\alpha-1)}(y) .
$$


It follows that,

$$
\begin{aligned}
K_{N, 4}^{(2,2)}(x, y) & =\frac{1}{2} \sum_{j=0}^{N-1} \varphi_{2 j+1}^{(\alpha-1)}(x) \widetilde{\varphi}_{2 j+1}^{(\alpha-1)}(y)+\frac{1}{2} \sum_{j=0}^{N} \varphi_{2 j}^{(\alpha-1)}(x) \widetilde{\varphi}_{2 j}^{(\alpha-1)}(y) \\
& -\frac{1}{2} \sqrt{\left(N+\frac{1}{2}\right)\left(N+\frac{\alpha}{2}\right)} \varepsilon \widetilde{\varphi}_{2 N+1}^{(\alpha-1)}(x) \widetilde{\varphi}_{2 N}^{(\alpha-1)}(y) \\
& =\frac{1}{2} \sum_{j=0}^{2 N} \varphi_{j}^{(\alpha-1)}(x) \widetilde{\varphi}_{j}^{(\alpha-1)}(y)-\frac{1}{2} \sqrt{\left(N+\frac{1}{2}\right)\left(N+\frac{\alpha}{2}\right)} \varepsilon \widetilde{\varphi}_{2 N+1}^{(\alpha-1)}(x) \widetilde{\varphi}_{2 N}^{(\alpha-1)}(y) \\
& =\frac{1}{2} S_{N}(x, y)-\frac{1}{2} \sqrt{\left(N+\frac{1}{2}\right)\left(N+\frac{\alpha}{2}\right)} \varepsilon \widetilde{\varphi}_{2 N+1}^{(\alpha-1)}(x) \widetilde{\varphi}_{2 N}^{(\alpha-1)}(y),
\end{aligned}
$$

where

$S_{N}(x, y):=\sum_{j=0}^{2 N} \varphi_{j}^{(\alpha-1)}(x) \widetilde{\varphi}_{j}^{(\alpha-1)}(y)=-\sqrt{(2 N+1)(2 N+\alpha)} \frac{\varphi_{2 N+1}^{(\alpha-1)}(x) \widetilde{\varphi}_{2 N}^{(\alpha-1)}(y)-\widetilde{\varphi}_{2 N+1}^{(\alpha-1)}(y) \varphi_{2 N}^{(\alpha-1)}(x)}{x-y}$.

Here we used the Christoffel-Darboux formula in the last equality.

By Theorem $[3.2$, we have the following theorem.

\section{Theorem 3.11.}

$$
\begin{aligned}
{\left[G_{N}^{(4)}(f)\right]^{2} } & =\operatorname{det}\left(I+S_{N} f-\frac{1}{2} S_{N} \varepsilon f^{\prime}-\sqrt{\left(N+\frac{1}{2}\right)\left(N+\frac{\alpha}{2}\right)}\left(\varepsilon \widetilde{\varphi}_{2 N+1}^{(\alpha-1)} \otimes \widetilde{\varphi}_{2 N}^{(\alpha-1)} f\right)\right. \\
& \left.-\frac{1}{2} \sqrt{\left(N+\frac{1}{2}\right)\left(N+\frac{\alpha}{2}\right)}\left(\varepsilon \widetilde{\varphi}_{2 N+1}^{(\alpha-1)} \otimes \varepsilon \widetilde{\varphi}_{2 N}^{(\alpha-1)}\right) f^{\prime}\right) .
\end{aligned}
$$

\subsection{Large $N$ behavior of the LSE moment generating function}

Now consider the scaling limit of $\left[G_{N}^{(4)}(f)\right]^{2}$, write

$$
\left[G_{N}^{(4)}(f)\right]^{2}=: \operatorname{det}(I+T)
$$

where

$$
\begin{aligned}
T: & =S_{N} f-\frac{1}{2} S_{N} \varepsilon f^{\prime}-\sqrt{\left(N+\frac{1}{2}\right)\left(N+\frac{\alpha}{2}\right)}\left(\varepsilon \widetilde{\varphi}_{2 N+1}^{(\alpha-1)} \otimes \widetilde{\varphi}_{2 N}^{(\alpha-1)} f\right) \\
& -\frac{1}{2} \sqrt{\left(N+\frac{1}{2}\right)\left(N+\frac{\alpha}{2}\right)}\left(\varepsilon \widetilde{\varphi}_{2 N+1}^{(\alpha-1)} \otimes \varepsilon \widetilde{\varphi}_{2 N}^{(\alpha-1)}\right) f^{\prime} .
\end{aligned}
$$

Theorem 3.12.

$$
\lim _{N \rightarrow \infty} \frac{y}{4 N} S_{N}\left(\frac{x^{2}}{8 N}, \frac{y^{2}}{8 N}\right)=B^{(\alpha-1)}(x, y)
$$




$$
\lim _{N \rightarrow \infty} \frac{x}{4 N} S_{N}\left(\frac{x^{2}}{8 N}, \frac{x^{2}}{8 N}\right)=B^{(\alpha-1)}(x, x),
$$

where $B^{(\alpha-1)}(x, y)$ and $B^{(\alpha-1)}(x, x)$ are given by (1.6) and (1.7) with $\alpha$ replaced by $\alpha-1$.

\section{Theorem 3.13.}

$$
\begin{gathered}
\widetilde{\varphi}_{2 N}^{(\alpha-1)}\left(\frac{x^{2}}{8 N}\right)=2(2 N)^{\frac{1}{2}} \frac{J_{\alpha-1}(x)}{x}+O\left(N^{-\frac{3}{2}}\right), N \rightarrow \infty, \\
\varepsilon \widetilde{\varphi}_{2 N}^{(\alpha-1)}\left(\frac{x^{2}}{8 N}\right)=(2 N)^{-\frac{1}{2}}\left[\int_{0}^{x} J_{\alpha-1}(y) d y-1\right]+O\left(N^{-\frac{5}{2}}\right), \quad N \rightarrow \infty, \\
\varepsilon \widetilde{\varphi}_{2 N+1}^{(\alpha-1)}\left(\frac{x^{2}}{8 N}\right)=(2 N)^{-\frac{1}{2}} \int_{0}^{x} J_{\alpha-1}(y) d y+O\left(N^{-\frac{5}{2}}\right), \quad N \rightarrow \infty .
\end{gathered}
$$

Proof. Recall the asymptotic formula of the Laguerre polynomials [13,

$L_{N}^{(\alpha)}(x) x^{\frac{\alpha}{2}} \mathrm{e}^{-\frac{x}{2}}=\frac{\Gamma(N+\alpha+1)}{\Gamma(N+1)}\left(N+\frac{\alpha+1}{2}\right)^{-\frac{\alpha}{2}} J_{\alpha}(\sqrt{(4 N+2 \alpha+2) x})+x^{\frac{5}{4}} O\left(N^{\frac{\alpha}{2}-\frac{3}{4}}\right), N \rightarrow \infty$.

We find,

$$
\begin{aligned}
\widetilde{\varphi}_{2 N}^{(\alpha-1)}\left(\frac{x^{2}}{8 N}\right) & =\sqrt{\frac{\Gamma(2 N+1)}{\Gamma(2 N+\alpha)}} L_{2 N}^{(\alpha-1)}\left(\frac{x^{2}}{8 N}\right)\left(\frac{x^{2}}{8 N}\right)^{\frac{\alpha}{2}-1} \mathrm{e}^{-\frac{x^{2}}{16 N}} \\
& =\sqrt{\frac{\Gamma(2 N+1)}{\Gamma(2 N+\alpha)}} \cdot\left(\frac{x^{2}}{8 N}\right)^{-\frac{1}{2}}\left[\frac{\Gamma(2 N+\alpha)}{\Gamma(2 N+1)}\left(2 N+\frac{\alpha}{2}\right)^{-\frac{\alpha-1}{2}} J_{\alpha-1}(x)+O\left(N^{\frac{\alpha}{2}-\frac{5}{2}}\right)\right] \\
& =2(2 N)^{\frac{1}{2}} \frac{J_{\alpha-1}(x)}{x}+O\left(N^{-\frac{3}{2}}\right), \quad N \rightarrow \infty
\end{aligned}
$$

where we have used the formula,

$$
\frac{\Gamma(n+a)}{\Gamma(n+b)}=n^{a-b}\left[1+O\left(n^{-1}\right)\right], n \rightarrow \infty .
$$

Proceeding to $\varepsilon \widetilde{\varphi}_{2 N}^{(\alpha-1)}(x)$, we have,

$$
\begin{aligned}
& \varepsilon \widetilde{\varphi}_{2 N}^{(\alpha-1)}(x) \\
= & \frac{1}{2}\left[\int_{0}^{x} \widetilde{\varphi}_{2 N}^{(\alpha-1)}(y) d y-\int_{x}^{\infty} \widetilde{\varphi}_{2 N}^{(\alpha-1)}(y) d y\right] \\
= & \frac{1}{2} \sqrt{\frac{\Gamma(2 N+1)}{\Gamma(2 N+\alpha)}\left[\int_{0}^{x} L_{2 N}^{(\alpha-1)}(y) y^{\frac{\alpha}{2}-1} \mathrm{e}^{-\frac{y}{2}} d y-\int_{x}^{\infty} L_{2 N}^{(\alpha-1)}(y) y^{\frac{\alpha}{2}-1} \mathrm{e}^{-\frac{y}{2}} d y\right]} \\
= & \frac{1}{2} \sqrt{\frac{\Gamma(2 N+1)}{\Gamma(2 N+\alpha)}}\left[2 \int_{0}^{x} L_{2 N}^{(\alpha-1)}(y) y^{\frac{\alpha}{2}-1} \mathrm{e}^{-\frac{y}{2}} d y-\int_{0}^{\infty} L_{2 N}^{(\alpha-1)}(y) y^{\frac{\alpha}{2}-1} \mathrm{e}^{-\frac{y}{2}} d y\right] \\
= & \frac{1}{2} \sqrt{\frac{\Gamma(2 N+1)}{\Gamma(2 N+\alpha)}}\left[2 \int_{0}^{x} L_{2 N}^{(\alpha-1)}(y) y^{\frac{\alpha}{2}-1} \mathrm{e}^{-\frac{y}{2}} d y-\frac{2^{\frac{\alpha}{2}} \Gamma\left(N+\frac{\alpha}{2}\right)}{\Gamma(N+1)}\right]
\end{aligned}
$$


where we have used the fact that

$$
\int_{0}^{\infty} L_{2 N}^{(\alpha-1)}(y) y^{\frac{\alpha}{2}-1} \mathrm{e}^{-\frac{y}{2}} d y=\frac{2^{\frac{\alpha}{2}} \Gamma\left(N+\frac{\alpha}{2}\right)}{\Gamma(N+1)} .
$$

Continuing,

$$
\begin{aligned}
& \varepsilon \widetilde{\varphi}_{2 N}^{(\alpha-1)}\left(\frac{x^{2}}{8 N}\right) \\
= & \frac{1}{2} \sqrt{\frac{\Gamma(2 N+1)}{\Gamma(2 N+\alpha)}}\left[\frac{1}{2 N} \int_{0}^{x} L_{2 N}^{(\alpha-1)}\left(\frac{y^{2}}{8 N}\right)\left(\frac{y^{2}}{8 N}\right)^{\frac{\alpha}{2}-1} \mathrm{e}^{-\frac{y^{2}}{16 N}} y d y-\frac{2^{\frac{\alpha}{2}} \Gamma\left(N+\frac{\alpha}{2}\right)}{\Gamma(N+1)}\right] \\
= & (2 N)^{-\frac{1}{2}}\left(2 N+\frac{\alpha}{2}\right)^{-\frac{\alpha-1}{2}} \sqrt{\frac{\Gamma(2 N+\alpha)}{\Gamma(2 N+1)}} \int_{0}^{x} J_{\alpha-1}(y) d y-2^{\frac{\alpha}{2}-1} \sqrt{\frac{\Gamma(2 N+1)}{\Gamma(2 N+\alpha)}} \cdot \frac{\Gamma\left(N+\frac{\alpha}{2}\right)}{\Gamma(N+1)}+O\left(N^{-\frac{5}{2}}\right) \\
= & (2 N)^{-\frac{1}{2}}\left[\int_{0}^{x} J_{\alpha-1}(y) d y-1\right]+O\left(N^{-\frac{5}{2}}\right), N \rightarrow \infty .
\end{aligned}
$$

Similarly,

$$
\begin{aligned}
& \varepsilon \widetilde{\varphi}_{2 N+1}^{(\alpha-1)}(x) \\
= & \frac{1}{2}\left[\int_{0}^{x} \widetilde{\varphi}_{2 N+1}^{(\alpha-1)}(y) d y-\int_{x}^{\infty} \widetilde{\varphi}_{2 N+1}^{(\alpha-1)}(y) d y\right] \\
= & \frac{1}{2} \sqrt{\frac{\Gamma(2 N+2)}{\Gamma(2 N+\alpha+1)}\left[\int_{0}^{x} L_{2 N+1}^{(\alpha-1)}(y) y^{\frac{\alpha}{2}-1} \mathrm{e}^{-\frac{y}{2}} d y-\int_{x}^{\infty} L_{2 N+1}^{(\alpha-1)}(y) y^{\frac{\alpha}{2}-1} \mathrm{e}^{-\frac{y}{2}} d y\right]} \\
= & \frac{1}{2} \sqrt{\frac{\Gamma(2 N+2)}{\Gamma(2 N+\alpha+1)}}\left[2 \int_{0}^{x} L_{2 N+1}^{(\alpha-1)}(y) y^{\frac{\alpha}{2}-1} \mathrm{e}^{-\frac{y}{2}} d y-\int_{0}^{\infty} L_{2 N+1}^{(\alpha-1)}(y) y^{\frac{\alpha}{2}-1} \mathrm{e}^{-\frac{y}{2}} d y\right] \\
= & \sqrt{\frac{\Gamma(2 N+2)}{\Gamma(2 N+\alpha+1)}} \int_{0}^{x} L_{2 N+1}^{(\alpha-1)}(y) y^{\frac{\alpha}{2}-1} \mathrm{e}^{-\frac{y}{2}} d y,
\end{aligned}
$$

where we have used the fact that

$$
\int_{0}^{\infty} L_{2 N+1}^{(\alpha-1)}(y) y^{\frac{\alpha}{2}-1} \mathrm{e}^{-\frac{y}{2}} d y=0 .
$$

It follows that

$$
\begin{aligned}
& \varepsilon \widetilde{\varphi}_{2 N+1}^{(\alpha-1)}\left(\frac{x^{2}}{8 N}\right) \\
= & \sqrt{\frac{\Gamma(2 N+2)}{\Gamma(2 N+\alpha+1)} \int_{0}^{\frac{x^{2}}{8 N}} L_{2 N+1}^{(\alpha-1)}(y) y^{\frac{\alpha}{2}-1} \mathrm{e}^{-\frac{y}{2}} d y} \\
= & \frac{1}{4 N} \sqrt{\frac{\Gamma(2 N+2)}{\Gamma(2 N+\alpha+1)}} \int_{0}^{x} L_{2 N+1}^{(\alpha-1)}\left(\frac{y^{2}}{8 N}\right)\left(\frac{y^{2}}{8 N}\right)^{\frac{\alpha}{2}-1} \mathrm{e}^{-\frac{y^{2}}{16 N}} y d y \\
= & (2 N)^{-\frac{1}{2}}\left(2 N+1+\frac{\alpha}{2}\right)^{-\frac{\alpha-1}{2}} \sqrt{\frac{\Gamma(2 N+\alpha+1)}{\Gamma(2 N+2)}} \int_{0}^{x} J_{\alpha-1}(y) d y+O\left(N^{-\frac{5}{2}}\right) \\
= & (2 N)^{-\frac{1}{2}} \int_{0}^{x} J_{\alpha-1}(y) d y+O\left(N^{-\frac{5}{2}}\right), N \rightarrow \infty
\end{aligned}
$$


We now use Theorem 3.12 and Theorem 3.13 to compute $\operatorname{Tr} T$ and $\operatorname{Tr} T^{2}$ as $N \rightarrow \infty$. In the computations below, we replace $f(x)$ by $f(\sqrt{8 N x})$ and $f^{\prime}(x)$ by

$$
f^{\prime}(\sqrt{8 N x})=\frac{d}{\sqrt{8 N} d \sqrt{x}} f(\sqrt{8 N x}) .
$$

Consider $\operatorname{Tr} T$, which reads,

$$
\begin{aligned}
\operatorname{Tr} T & =\operatorname{Tr} S_{N} f-\operatorname{Tr} \frac{1}{2} S_{N} \varepsilon f^{\prime}-\operatorname{Tr} \sqrt{\left(N+\frac{1}{2}\right)\left(N+\frac{\alpha}{2}\right)}\left(\varepsilon \widetilde{\varphi}_{2 N+1}^{(\alpha-1)} \otimes \widetilde{\varphi}_{2 N}^{(\alpha-1)} f\right) \\
& -\operatorname{Tr} \frac{1}{2} \sqrt{\left(N+\frac{1}{2}\right)\left(N+\frac{\alpha}{2}\right)}\left(\varepsilon \widetilde{\varphi}_{2 N+1}^{(\alpha-1)} \otimes \varepsilon \widetilde{\varphi}_{2 N}^{(\alpha-1)}\right) f^{\prime} .
\end{aligned}
$$

So we compute $\operatorname{Tr} T$ by calculating the four terms in the right side. The first term,

$$
\begin{aligned}
\operatorname{Tr} S_{N} f & =\int_{0}^{\infty} S_{N}(x, x) f(\sqrt{8 N x}) d x \\
& =\int_{0}^{\infty} \frac{x}{4 N} S_{N}\left(\frac{x^{2}}{8 N}, \frac{x^{2}}{8 N}\right) f(x) d x \\
& \rightarrow \int_{0}^{\infty} B^{(\alpha-1)}(x, x) f(x) d x, \quad N \rightarrow \infty
\end{aligned}
$$

The second term,

$$
\begin{aligned}
\operatorname{Tr} \frac{1}{2} S_{N} \varepsilon f^{\prime} & =\frac{1}{2} \int_{0}^{\infty} \int_{0}^{\infty} S_{N}(x, y) \varepsilon(y, x) f^{\prime}(\sqrt{8 N x}) d x d y \\
& =\frac{1}{4} \int_{0}^{\infty}\left[\int_{x}^{\infty} S_{N}(x, y) d y-\int_{0}^{x} S_{N}(x, y) d y\right] f^{\prime}(\sqrt{8 N x}) d x
\end{aligned}
$$

Let

$$
u=\sqrt{8 N x}, \quad v=\sqrt{8 N y}
$$

then

$$
\begin{aligned}
\operatorname{Tr} \frac{1}{2} S_{N} \varepsilon f^{\prime} & =\frac{1}{16 N} \int_{0}^{\infty}\left[\int_{u}^{\infty} \frac{1}{4 N} S_{N}\left(\frac{u^{2}}{8 N}, \frac{v^{2}}{8 N}\right) v d v-\int_{0}^{u} \frac{1}{4 N} S_{N}\left(\frac{u^{2}}{8 N}, \frac{v^{2}}{8 N}\right) v d v\right] u f^{\prime}(u) d u \\
& \rightarrow \frac{1}{16 N} \int_{0}^{\infty}\left[\int_{u}^{\infty} B^{(\alpha-1)}(u, v) d v-\int_{0}^{u} B^{(\alpha-1)}(u, v) d v\right] u f^{\prime}(u) d u, \quad N \rightarrow \infty .
\end{aligned}
$$


The third term,

$$
\begin{aligned}
& \operatorname{Tr} \sqrt{\left(N+\frac{1}{2}\right)\left(N+\frac{\alpha}{2}\right)}\left(\varepsilon \widetilde{\varphi}_{2 N+1}^{(\alpha-1)} \otimes \widetilde{\varphi}_{2 N}^{(\alpha-1)} f\right) \\
= & \sqrt{\left(N+\frac{1}{2}\right)\left(N+\frac{\alpha}{2}\right)} \int_{0}^{\infty} \varepsilon \widetilde{\varphi}_{2 N+1}^{(\alpha-1)}(x) \widetilde{\varphi}_{2 N}^{(\alpha-1)}(x) f(\sqrt{8 N x}) d x \\
= & \frac{\sqrt{\left(N+\frac{1}{2}\right)\left(N+\frac{\alpha}{2}\right)}}{4 N} \int_{0}^{\infty} \varepsilon \widetilde{\varphi}_{2 N+1}^{(\alpha-1)}\left(\frac{x^{2}}{8 N}\right) \widetilde{\varphi}_{2 N}^{(\alpha-1)}\left(\frac{x^{2}}{8 N}\right) x f(x) d x \\
= & \frac{1}{2} \int_{0}^{\infty}\left[\int_{0}^{x} J_{\alpha-1}(y) d y\right] J_{\alpha-1}(x) f(x) d x+O\left(N^{-2}\right), \quad N \rightarrow \infty .
\end{aligned}
$$

The fourth term,

$$
\begin{aligned}
& \operatorname{Tr} \frac{1}{2} \sqrt{\left(N+\frac{1}{2}\right)\left(N+\frac{\alpha}{2}\right)}\left(\varepsilon \widetilde{\varphi}_{2 N+1}^{(\alpha-1)} \otimes \varepsilon \widetilde{\varphi}_{2 N}^{(\alpha-1)}\right) f^{\prime} \\
= & \frac{1}{2} \sqrt{\left(N+\frac{1}{2}\right)\left(N+\frac{\alpha}{2}\right)} \int_{0}^{\infty} \varepsilon \widetilde{\varphi}_{2 N+1}^{(\alpha-1)}(x) \varepsilon \widetilde{\varphi}_{2 N}^{(\alpha-1)}(x) f^{\prime}(\sqrt{8 N x}) d x \\
= & \frac{\sqrt{\left(N+\frac{1}{2}\right)\left(N+\frac{\alpha}{2}\right)}}{8 N} \int_{0}^{\infty} \varepsilon \widetilde{\varphi}_{2 N+1}^{(\alpha-1)}\left(\frac{x^{2}}{8 N}\right) \varepsilon \widetilde{\varphi}_{2 N}^{(\alpha-1)}\left(\frac{x^{2}}{8 N}\right) x f^{\prime}(x) d x \\
= & \frac{1}{16 N} \int_{0}^{\infty}\left[\int_{0}^{x} J_{\alpha-1}(y) d y\right]\left[\int_{0}^{x} J_{\alpha-1}(y) d y-1\right] x f^{\prime}(x) d x+O\left(N^{-3}\right), \quad N \rightarrow \infty .
\end{aligned}
$$

Therefore,

$$
\begin{aligned}
\operatorname{Tr} T & =\int_{0}^{\infty} B^{(\alpha-1)}(x, x) f(x) d x-\frac{1}{2} \int_{0}^{\infty}\left[\int_{0}^{x} J_{\alpha-1}(y) d y\right] J_{\alpha-1}(x) f(x) d x \\
& -\frac{1}{16 N} \int_{0}^{\infty}\left[\int_{x}^{\infty} B^{(\alpha-1)}(x, y) d y-\int_{0}^{x} B^{(\alpha-1)}(x, y) d y\right] x f^{\prime}(x) d x \\
& -\frac{1}{16 N} \int_{0}^{\infty}\left[\int_{0}^{x} J_{\alpha-1}(y) d y\right]\left[\int_{0}^{x} J_{\alpha-1}(y) d y-1\right] x f^{\prime}(x) d x+O\left(N^{-2}\right), \quad N \rightarrow \infty
\end{aligned}
$$


Next, we compute $\operatorname{Tr} T^{2}$, where there are 10 traces,

$$
\begin{aligned}
\operatorname{Tr} T^{2} & =\operatorname{Tr} S_{N} f S_{N} f-\operatorname{Tr} S_{N} f S_{N} \varepsilon f^{\prime}-\operatorname{Tr} 2 \sqrt{\left(N+\frac{1}{2}\right)\left(N+\frac{\alpha}{2}\right)} S_{N} f\left(\varepsilon \widetilde{\varphi}_{2 N+1}^{(\alpha-1)} \otimes \widetilde{\varphi}_{2 N}^{(\alpha-1)} f\right) \\
& -\operatorname{Tr} \sqrt{\left(N+\frac{1}{2}\right)\left(N+\frac{\alpha}{2}\right)} S_{N} f\left(\varepsilon \widetilde{\varphi}_{2 N+1}^{(\alpha-1)} \otimes \varepsilon \widetilde{\varphi}_{2 N}^{(\alpha-1)}\right) f^{\prime}+\operatorname{Tr} \frac{1}{4} S_{N} \varepsilon f^{\prime} S_{N} \varepsilon f^{\prime} \\
& +\operatorname{Tr} \sqrt{\left(N+\frac{1}{2}\right)\left(N+\frac{\alpha}{2}\right)} S_{N} \varepsilon f^{\prime}\left(\varepsilon \widetilde{\varphi}_{2 N+1}^{(\alpha-1)} \otimes \widetilde{\varphi}_{2 N}^{(\alpha-1)} f\right) \\
& +\operatorname{Tr} \frac{1}{2} \sqrt{\left(N+\frac{1}{2}\right)\left(N+\frac{\alpha}{2}\right)} S_{N} \varepsilon f^{\prime}\left(\varepsilon \widetilde{\varphi}_{2 N+1}^{(\alpha-1)} \otimes \varepsilon \widetilde{\varphi}_{2 N}^{(\alpha-1)}\right) f^{\prime} \\
& +\operatorname{Tr}\left(N+\frac{1}{2}\right)\left(N+\frac{\alpha}{2}\right)\left(\varepsilon \widetilde{\varphi}_{2 N+1}^{(\alpha-1)} \otimes \widetilde{\varphi}_{2 N}^{(\alpha-1)} f\right)\left(\varepsilon \widetilde{\varphi}_{2 N+1}^{(\alpha-1)} \otimes \widetilde{\varphi}_{2 N}^{(\alpha-1)} f\right) \\
& +\operatorname{Tr}\left(N+\frac{1}{2}\right)\left(N+\frac{\alpha}{2}\right)\left(\varepsilon \widetilde{\varphi}_{2 N+1}^{(\alpha-1)} \otimes \widetilde{\varphi}_{2 N}^{(\alpha-1)} f\right)\left(\varepsilon \widetilde{\varphi}_{2 N+1}^{(\alpha-1)} \otimes \varepsilon \widetilde{\varphi}_{2 N}^{(\alpha-1)}\right) f^{\prime} \\
& +\operatorname{Tr} \frac{1}{4}\left(N+\frac{1}{2}\right)\left(N+\frac{\alpha}{2}\right)\left(\varepsilon \widetilde{\varphi}_{2 N+1}^{(\alpha-1)} \otimes \varepsilon \widetilde{\varphi}_{2 N}^{(\alpha-1)}\right) f^{\prime}\left(\varepsilon \widetilde{\varphi}_{2 N+1}^{(\alpha-1)} \otimes \varepsilon \widetilde{\varphi}_{2 N}^{(\alpha-1)}\right) f^{\prime} .
\end{aligned}
$$

In the following, we need to calculate the traces on the right side term by term. The first term,

$$
\begin{aligned}
\operatorname{Tr} S_{N} f S_{N} f & =\int_{0}^{\infty} \int_{0}^{\infty} S_{N}(x, y) f(\sqrt{8 N y}) S_{N}(y, x) f(\sqrt{8 N x}) d x d y \\
& =\int_{0}^{\infty} \int_{0}^{\infty} \frac{y}{4 N} S_{N}\left(\frac{x^{2}}{8 N}, \frac{y^{2}}{8 N}\right) \frac{x}{4 N} S_{N}\left(\frac{y^{2}}{8 N}, \frac{x^{2}}{8 N}\right) f(x) f(y) d x d y \\
& \rightarrow \int_{0}^{\infty} \int_{0}^{\infty} B^{(\alpha-1)}(x, y) B^{(\alpha-1)}(y, x) f(x) f(y) d x d y, \quad N \rightarrow \infty .
\end{aligned}
$$

The second term,

$\operatorname{Tr} S_{N} f S_{N} \varepsilon f^{\prime}$

$$
\begin{aligned}
= & \frac{1}{8 N} \int_{0}^{\infty} \int_{0}^{\infty} \frac{v}{4 N} S_{N}\left(\frac{u^{2}}{8 N}, \frac{v^{2}}{8 N}\right)\left[\int_{u}^{\infty} \frac{w}{4 N} S_{N}\left(\frac{v^{2}}{8 N}, \frac{w^{2}}{8 N}\right) d w-\int_{0}^{u} \frac{w}{4 N} S_{N}\left(\frac{v^{2}}{8 N}, \frac{w^{2}}{8 N}\right) d w\right] \\
& u f^{\prime}(u) f(v) d u d v \\
\rightarrow & \frac{1}{8 N} \int_{0}^{\infty} \int_{0}^{\infty} B^{(\alpha-1)}(u, v)\left[\int_{u}^{\infty} B^{(\alpha-1)}(v, w) d w-\int_{0}^{u} B^{(\alpha-1)}(v, w) d w\right] u f^{\prime}(u) f(v) d u d v, \quad N \rightarrow \infty .
\end{aligned}
$$

The third term,

$$
\begin{aligned}
& \operatorname{Tr} 2 \sqrt{\left(N+\frac{1}{2}\right)\left(N+\frac{\alpha}{2}\right)} S_{N} f\left(\varepsilon \widetilde{\varphi}_{2 N+1}^{(\alpha-1)} \otimes \widetilde{\varphi}_{2 N}^{(\alpha-1)} f\right) \\
= & \frac{1}{2 N} \sqrt{\left(N+\frac{1}{2}\right)\left(N+\frac{\alpha}{2}\right)} \int_{0}^{\infty} \int_{0}^{\infty} \frac{1}{4 N} S_{N}\left(\frac{u^{2}}{8 N}, \frac{v^{2}}{8 N}\right) v \widetilde{\varphi}_{2 N}^{(\alpha-1)}\left(\frac{u^{2}}{8 N}\right) \varepsilon \widetilde{\varphi}_{2 N+1}^{(\alpha-1)}\left(\frac{v^{2}}{8 N}\right) u f(u) f(v) d u d v \\
= & \int_{0}^{\infty} \int_{0}^{\infty} B^{(\alpha-1)}(u, v) J_{\alpha-1}(u)\left[\int_{0}^{v} J_{\alpha-1}(t) d t\right] f(u) f(v) d u d v+O\left(N^{-2}\right), \quad N \rightarrow \infty
\end{aligned}
$$


The fourth term,

$$
\begin{aligned}
& \operatorname{Tr} \sqrt{\left(N+\frac{1}{2}\right)\left(N+\frac{\alpha}{2}\right)} S_{N} f\left(\varepsilon \widetilde{\varphi}_{2 N+1}^{(\alpha-1)} \otimes \varepsilon \widetilde{\varphi}_{2 N}^{(\alpha-1)}\right) f^{\prime} \\
= & \frac{1}{4 N} \sqrt{\left(N+\frac{1}{2}\right)\left(N+\frac{\alpha}{2}\right)} \int_{0}^{\infty} \int_{0}^{\infty} \frac{1}{4 N} S_{N}\left(\frac{u^{2}}{8 N}, \frac{v^{2}}{8 N}\right) v f(v) \varepsilon \widetilde{\varphi}_{2 N+1}^{(\alpha-1)}\left(\frac{v^{2}}{8 N}\right) \varepsilon \widetilde{\varphi}_{2 N}^{(\alpha-1)}\left(\frac{u^{2}}{8 N}\right) u f^{\prime}(u) d u d v \\
= & \frac{1}{8 N} \int_{0}^{\infty} \int_{0}^{\infty} B^{(\alpha-1)}(u, v)\left[\int_{0}^{u} J_{\alpha-1}(t) d t-1\right]\left[\int_{0}^{v} J_{\alpha-1}(t) d t\right] u f^{\prime}(u) f(v) d u d v+O\left(N^{-3}\right), \quad N \rightarrow \infty
\end{aligned}
$$

The fifth term,

$$
\begin{aligned}
& \operatorname{Tr} \frac{1}{4} S_{N} \varepsilon f^{\prime} S_{N} \varepsilon f^{\prime} \\
= & \frac{1}{4} \int_{0}^{\infty} \int_{0}^{\infty} \int_{0}^{\infty} \int_{0}^{\infty} S_{N}(x, y) \varepsilon(y, z) f^{\prime}(\sqrt{8 N z}) S_{N}(z, t) \varepsilon(t, x) f^{\prime}(\sqrt{8 N x}) d x d y d z d t \\
= & \frac{1}{16} \int_{0}^{\infty} \int_{0}^{\infty}\left[\int_{z}^{\infty} S_{N}(x, y) d y-\int_{0}^{z} S_{N}(x, y) d y\right]\left[\int_{x}^{\infty} S_{N}(z, t) d t-\int_{0}^{x} S_{N}(z, t) d t\right] \\
= & \frac{1}{256 N^{2}} \int_{0}^{\infty} \int_{0}^{\infty}\left[\int_{w}^{\infty} \frac{1}{4 N} S_{N}\left(\frac{u^{2}}{8 N}, \frac{v^{2}}{8 N}\right) v d v-\int_{0}^{w} \frac{1}{4 N} S_{N}\left(\frac{u^{2}}{8 N}, \frac{v^{2}}{8 N}\right) v d v\right] \\
& {\left[\int_{u}^{\infty} \frac{1}{4 N} S_{N}\left(\frac{w^{2}}{8 N}, \frac{\tau^{2}}{8 N}\right) \tau d \tau-\int_{0}^{u} \frac{1}{4 N} S_{N}\left(\frac{w^{2}}{8 N}, \frac{\tau^{2}}{8 N}\right) \tau d \tau\right] u w f^{\prime}(u) f^{\prime}(w) d u d w } \\
= & O\left(\frac{1}{N^{2}}\right), N \rightarrow \infty .
\end{aligned}
$$

The sixth term,

$$
\begin{aligned}
& \operatorname{Tr} \sqrt{\left(N+\frac{1}{2}\right)\left(N+\frac{\alpha}{2}\right)} S_{N} \varepsilon f^{\prime}\left(\varepsilon \widetilde{\varphi}_{2 N+1}^{(\alpha-1)} \otimes \widetilde{\varphi}_{2 N}^{(\alpha-1)} f\right) \\
= & \frac{1}{32 N^{2}} \sqrt{\left(N+\frac{1}{2}\right)\left(N+\frac{\alpha}{2}\right)} \int_{0}^{\infty} \int_{0}^{\infty}\left[\int_{w}^{\infty} \frac{v}{4 N} S_{N}\left(\frac{u^{2}}{8 N}, \frac{v^{2}}{8 N}\right) d v-\int_{0}^{w} \frac{v}{4 N} S_{N}\left(\frac{u^{2}}{8 N}, \frac{v^{2}}{8 N}\right) d v\right] \\
& f^{\prime}(w) \varepsilon \widetilde{\varphi}_{2 N+1}^{(\alpha-1)}\left(\frac{w^{2}}{8 N}\right) \widetilde{\varphi}_{2 N}^{(\alpha-1)}\left(\frac{u^{2}}{8 N}\right) u w f(u) d u d w \\
= & \frac{1}{16 N} \int_{0}^{\infty} \int_{0}^{\infty}\left[\int_{w}^{\infty} B^{(\alpha-1)}(u, v) d v-\int_{0}^{w} B^{(\alpha-1)}(u, v) d v\right]\left[\int_{0}^{w} J_{\alpha-1}(t) d t\right] J_{\alpha-1}(u) w f^{\prime}(w) f(u) d u d w \\
+ & O\left(N^{-3}\right), \quad N \rightarrow \infty .
\end{aligned}
$$


The seventh term,

$$
\begin{aligned}
& \operatorname{Tr} \frac{1}{2} \sqrt{\left(N+\frac{1}{2}\right)\left(N+\frac{\alpha}{2}\right)} S_{N} \varepsilon f^{\prime}\left(\varepsilon \widetilde{\varphi}_{2 N+1}^{(\alpha-1)} \otimes \varepsilon \widetilde{\varphi}_{2 N}^{(\alpha-1)}\right) f^{\prime} \\
= & \frac{1}{64 N^{2}} \sqrt{\left(N+\frac{1}{2}\right)\left(N+\frac{\alpha}{2}\right)} \int_{0}^{\infty} \int_{0}^{\infty}\left[\int_{w}^{\infty} \frac{v}{4 N} S_{N}\left(\frac{u^{2}}{8 N}, \frac{v^{2}}{8 N}\right) d v-\int_{0}^{w} \frac{v}{4 N} S_{N}\left(\frac{u^{2}}{8 N}, \frac{v^{2}}{8 N}\right) d v\right] \\
& f^{\prime}(w) \varepsilon \widetilde{\varphi}_{2 N+1}^{(\alpha-1)}\left(\frac{w^{2}}{8 N}\right) \varepsilon \widetilde{\varphi}_{2 N}^{(\alpha-1)}\left(\frac{u^{2}}{8 N}\right) f^{\prime}(u) u w d u d w \\
= & O\left(\frac{1}{N^{2}}\right), N \rightarrow \infty .
\end{aligned}
$$

The eighth term,

$$
\begin{aligned}
& \operatorname{Tr}\left(N+\frac{1}{2}\right)\left(N+\frac{\alpha}{2}\right)\left(\varepsilon \widetilde{\varphi}_{2 N+1}^{(\alpha-1)} \otimes \widetilde{\varphi}_{2 N}^{(\alpha-1)} f\right)\left(\varepsilon \widetilde{\varphi}_{2 N+1}^{(\alpha-1)} \otimes \widetilde{\varphi}_{2 N}^{(\alpha-1)} f\right) \\
= & \frac{\left(N+\frac{1}{2}\right)\left(N+\frac{\alpha}{2}\right)}{16 N^{2}} \int_{0}^{\infty} \int_{0}^{\infty} \varepsilon \widetilde{\varphi}_{2 N+1}^{(\alpha-1)}\left(\frac{u^{2}}{8 N}\right) \varepsilon \widetilde{\varphi}_{2 N+1}^{(\alpha-1)}\left(\frac{v^{2}}{8 N}\right) \widetilde{\varphi}_{2 N}^{(\alpha-1)}\left(\frac{u^{2}}{8 N}\right) \widetilde{\varphi}_{2 N}^{(\alpha-1)}\left(\frac{v^{2}}{8 N}\right) u v f(u) f(v) d u d v \\
= & \frac{1}{4} \int_{0}^{\infty} \int_{0}^{\infty}\left[\int_{0}^{u} J_{\alpha-1}(t) d t\right]\left[\int_{0}^{v} J_{\alpha-1}(t) d t\right] J_{\alpha-1}(u) J_{\alpha-1}(v) f(u) f(v) d u d v+O\left(N^{-2}\right), N \rightarrow \infty .
\end{aligned}
$$

The ninth term,

$$
\begin{aligned}
& \operatorname{Tr}\left(N+\frac{1}{2}\right)\left(N+\frac{\alpha}{2}\right)\left(\varepsilon \widetilde{\varphi}_{2 N+1}^{(\alpha-1)} \otimes \widetilde{\varphi}_{2 N}^{(\alpha-1)} f\right)\left(\varepsilon \widetilde{\varphi}_{2 N+1}^{(\alpha-1)} \otimes \varepsilon \widetilde{\varphi}_{2 N}^{(\alpha-1)}\right) f^{\prime} \\
= & \frac{\left(N+\frac{1}{2}\right)\left(N+\frac{\alpha}{2}\right)}{16 N^{2}} \int_{0}^{\infty} \int_{0}^{\infty} \varepsilon \widetilde{\varphi}_{2 N+1}^{(\alpha-1)}\left(\frac{u^{2}}{8 N}\right) \varepsilon \widetilde{\varphi}_{2 N+1}^{(\alpha-1)}\left(\frac{v^{2}}{8 N}\right) \varepsilon \widetilde{\varphi}_{2 N}^{(\alpha-1)}\left(\frac{u^{2}}{8 N}\right) \widetilde{\varphi}_{2 N}^{(\alpha-1)}\left(\frac{v^{2}}{8 N}\right) \\
& u v f^{\prime}(u) f(v) d u d v \\
= & \frac{1}{16 N} \int_{0}^{\infty} \int_{0}^{\infty}\left[\int_{0}^{u} J_{\alpha-1}(t) d t\right]\left[\int_{0}^{v} J_{\alpha-1}(t) d t\right]\left[\int_{0}^{u} J_{\alpha-1}(t) d t-1\right] J_{\alpha-1}(v) u f^{\prime}(u) f(v) d u d v \\
+ & O\left(N^{-3}\right), N \rightarrow \infty .
\end{aligned}
$$

The tenth term,

$$
\begin{aligned}
& \operatorname{Tr} \frac{1}{4}\left(N+\frac{1}{2}\right)\left(N+\frac{\alpha}{2}\right)\left(\varepsilon \widetilde{\varphi}_{2 N+1}^{(\alpha-1)} \otimes \varepsilon \widetilde{\varphi}_{2 N}^{(\alpha-1)}\right) f^{\prime}\left(\varepsilon \widetilde{\varphi}_{2 N+1}^{(\alpha-1)} \otimes \varepsilon \widetilde{\varphi}_{2 N}^{(\alpha-1)}\right) f^{\prime} \\
= & \frac{1}{64 N^{2}}\left(N+\frac{1}{2}\right)\left(N+\frac{\alpha}{2}\right) \int_{0}^{\infty} \int_{0}^{\infty} \varepsilon \widetilde{\varphi}_{2 N+1}^{(\alpha-1)}\left(\frac{u^{2}}{8 N}\right) \varepsilon \widetilde{\varphi}_{2 N+1}^{(\alpha-1)}\left(\frac{v^{2}}{8 N}\right) \varepsilon \widetilde{\varphi}_{2 N}^{(\alpha-1)}\left(\frac{u^{2}}{8 N}\right) \varepsilon \widetilde{\varphi}_{2 N}^{(\alpha-1)}\left(\frac{v^{2}}{8 N}\right) \\
& u v f^{\prime}(u) f^{\prime}(v) d u d v \\
= & O\left(\frac{1}{N^{2}}\right), N \rightarrow \infty .
\end{aligned}
$$


Therefore,

$\operatorname{Tr} T^{2}$

$$
\begin{aligned}
& =\int_{0}^{\infty} \int_{0}^{\infty} B^{(\alpha-1)}(x, y) B^{(\alpha-1)}(y, x) f(x) f(y) d x d y \\
& -\int_{0}^{\infty} \int_{0}^{\infty} B^{(\alpha-1)}(x, y) J_{\alpha-1}(x)\left[\int_{0}^{y} J_{\alpha-1}(z) d z\right] f(x) f(y) d x d y \\
& +\frac{1}{4} \int_{0}^{\infty} \int_{0}^{\infty}\left[\int_{0}^{x} J_{\alpha-1}(z) d z\right]\left[\int_{0}^{y} J_{\alpha-1}(z) d z\right] J_{\alpha-1}(x) J_{\alpha-1}(y) f(x) f(y) d x d y \\
& -\frac{1}{8 N} \int_{0}^{\infty} \int_{0}^{\infty} B^{(\alpha-1)}(x, y)\left[\int_{x}^{\infty} B^{(\alpha-1)}(y, z) d z-\int_{0}^{x} B^{(\alpha-1)}(y, z) d z\right] x f^{\prime}(x) f(y) d x d y \\
& -\frac{1}{8 N} \int_{0}^{\infty} \int_{0}^{\infty} B^{(\alpha-1)}(x, y)\left[\int_{0}^{x} J_{\alpha-1}(z) d z-1\right]\left[\int_{0}^{y} J_{\alpha-1}(z) d z\right] x f^{\prime}(x) f(y) d x d y \\
& +\frac{1}{16 N} \int_{0}^{\infty} \int_{0}^{\infty}\left[\int_{x}^{\infty} B^{(\alpha-1)}(y, z) d z-\int_{0}^{x} B^{(\alpha-1)}(y, z) d z\right]\left[\int_{0}^{x} J_{\alpha-1}(z) d z\right] J_{\alpha-1}(y) x f^{\prime}(x) f(y) d x d y \\
& +\frac{1}{16 N} \int_{0}^{\infty} \int_{0}^{\infty}\left[\int_{0}^{x} J_{\alpha-1}(z) d z\right]\left[\int_{0}^{y} J_{\alpha-1}(z) d z\right]\left[\int_{0}^{x} J_{\alpha-1}(z) d z-1\right] J_{\alpha-1}(y) x f^{\prime}(x) f(y) d x d y \\
& +O(N-2), N \rightarrow \infty .
\end{aligned}
$$

Now we want to see the mean and variance of the linear statistics $\sum_{j=1}^{N} F\left(\sqrt{8 N x_{j}}\right)$, so we need to obtain the coefficients of $\lambda$ and $\lambda^{2}$, firstly we know

$$
f(\sqrt{8 N x}) \approx-\lambda F(\sqrt{8 N x})+\frac{\lambda^{2}}{2} F^{2}(\sqrt{8 N x})
$$

then we replace $f$ with $-\lambda F+\frac{\lambda^{2}}{2} F^{2}$ in the expression of $\operatorname{Tr} T$ and $\operatorname{Tr} T^{2}$, similar to previous discussions, denote by $\mu_{N}^{(L S E, \alpha)}$ and $\mathcal{V}_{N}^{(L S E, \alpha)}$ the mean and variance of the linear statistics $\sum_{j=1}^{N} F\left(\sqrt{8 N x_{j}}\right)$, we have the following theorem.

Theorem 3.14. As $N \rightarrow \infty$,

$$
\begin{aligned}
\mu_{N}^{(L S E, \alpha)} & =\frac{1}{2} \mu_{N}^{(L U E, \alpha-1)}-\frac{1}{4} \int_{0}^{\infty}\left[\int_{0}^{x} J_{\alpha-1}(y) d y\right] J_{\alpha-1}(x) F(x) d x \\
& -\frac{1}{32 N} \int_{0}^{\infty}\left[\int_{x}^{\infty} B^{(\alpha-1)}(x, y) d y-\int_{0}^{x} B^{(\alpha-1)}(x, y) d y\right] x F^{\prime}(x) d x \\
& -\frac{1}{32 N} \int_{0}^{\infty}\left[\int_{0}^{x} J_{\alpha-1}(y) d y\right]\left[\int_{0}^{x} J_{\alpha-1}(y) d y-1\right] x F^{\prime}(x) d x+O\left(N^{-2}\right)
\end{aligned}
$$




$$
\begin{aligned}
\mathcal{V}_{N}^{(L S E, \alpha)} & =\frac{1}{2} \mathcal{V}_{N}^{(L U E, \alpha-1)}-\frac{1}{4} \int_{0}^{\infty}\left[\int_{0}^{x} J_{\alpha-1}(y) d y\right] J_{\alpha-1}(x) F^{2}(x) d x \\
& +\frac{1}{2} \int_{0}^{\infty} \int_{0}^{\infty} B^{(\alpha-1)}(x, y) J_{\alpha-1}(x)\left[\int_{0}^{y} J_{\alpha-1}(z) d z\right] F(x) F(y) d x d y \\
& -\frac{1}{8} \int_{0}^{\infty} \int_{0}^{\infty}\left[\int_{0}^{x} J_{\alpha-1}(z) d z\right]\left[\int_{0}^{y} J_{\alpha-1}(z) d z\right] J_{\alpha-1}(x) J_{\alpha-1}(y) F(x) F(y) d x d y \\
& -\frac{1}{16 N} \int_{0}^{\infty}\left[\int_{x}^{\infty} B^{(\alpha-1)}(x, y) d y-\int_{0}^{x} B^{(\alpha-1)}(x, y) d y\right] x F(x) F^{\prime}(x) d x \\
& -\frac{1}{16 N} \int_{0}^{\infty}\left[\int_{0}^{x} J_{\alpha-1}(y) d y\right]\left[\int_{0}^{x} J_{\alpha-1}(y) d y-1\right] x F(x) F^{\prime}(x) d x \\
+ & \frac{1}{16 N} \int_{0}^{\infty} \int_{0}^{\infty} B^{(\alpha-1)}(x, y)\left[\int_{x}^{\infty} B^{(\alpha-1)}(y, z) d z-\int_{0}^{x} B^{(\alpha-1)}(y, z) d z\right] x F^{\prime}(x) F(y) d x d y \\
+ & \frac{1}{16 N} \int_{0}^{\infty} \int_{0}^{\infty} B^{(\alpha-1)}(x, y)\left[\int_{0}^{x} J_{\alpha-1}(z) d z-1\right]\left[\int_{0}^{y} J_{\alpha-1}(z) d z\right] x F^{\prime}(x) F(y) d x d y \\
& -\frac{1}{32 N} \int_{0}^{\infty} \int_{0}^{\infty}\left[\int_{x}^{\infty} B^{(\alpha-1)}(y, z) d z-\int_{0}^{x} B^{(\alpha-1)}(y, z) d z\right]\left[\int_{0}^{x} J_{\alpha-1}(z) d z\right] J_{\alpha-1}(y) \\
& x F^{\prime}(x) F(y) d x d y \\
& \frac{1}{32 N} \int_{0}^{\infty} \int_{0}^{\infty}\left[\int_{0}^{x} J_{\alpha-1}(z) d z\right]\left[\int_{0}^{y} J_{\alpha-1}(z) d z\right]\left[\int_{0}^{x} J_{\alpha-1}(z) d z-1\right] J_{\alpha-1}(y) x F^{\prime}(x) F(y) d x d y \\
+ & O\left(N^{-2}\right)
\end{aligned}
$$

where $\mu_{N}^{(L U E, \alpha-1)}$ and $\mathcal{V}_{N}^{(L U E, \alpha-1)}$ for $N \rightarrow \infty$ are given in (1.4) and (1.5) respectively with $\alpha$ replaced by $\alpha-1$.

\section{The orthogonal ensembles}

\subsection{General case}

For the orthogonal ensembles, $\beta=1$, the equation (1.1), becomes,

$$
G_{N}^{(1)}(f)=C_{N}^{(1)} \int_{[a, b]^{n}} \prod_{1 \leq j<k \leq N}\left|x_{j}-x_{k}\right| \prod_{j=1}^{N} w\left(x_{j}\right)\left[1+f\left(x_{j}\right)\right] d x_{j},
$$

and we assume $N$ is even. Here,

$$
C_{N}^{(1)}=\frac{1}{\int_{(a, b)^{N}} \prod_{1 \leq j<k \leq N}\left|x_{j}-x_{k}\right| \prod_{j=1}^{N} w\left(x_{j}\right) d x_{j}}
$$

depends on $N$. 
We also follow the treatment of [6, 15], firstly using Theorem 2.5 and some computations, we find

$$
\left[G_{N}^{(1)}(f)\right]^{2}=\widehat{C_{N}^{(1)}} \operatorname{det}\left(\int_{a}^{b} \int_{a}^{b} \varepsilon(x, y) \pi_{j}(x) \pi_{k}(y) w(x) w(y)(1+f(x))(1+f(y)) d x d y\right)_{j, k=0}^{N-1}
$$

where $\widehat{C_{N}^{(1)}}$ is a constant depending on $N$ and $\pi_{j}(x)$ is an arbitrary polynomial of degree $j$. Let

$$
\psi_{j}(x)=\pi_{j}(x) w(x)
$$

it follows that

$$
\left[G_{N}^{(1)}(f)\right]^{2}=\operatorname{det}\left(I+\left(M^{(1)}\right)^{-1} L^{(1)}\right)
$$

where

$$
\begin{gathered}
M^{(1)}=\left(\int_{a}^{b} \psi_{j}(x) \varepsilon \psi_{k}(x) d x\right)_{j, k=0}^{N-1}, \\
L^{(1)}=\left(\int_{a}^{b}\left(f(x) \psi_{j}(x) \varepsilon \psi_{k}(x)-f(x) \psi_{k}(x) \varepsilon \psi_{j}(x)-f(x) \psi_{k}(x) \varepsilon\left(f \psi_{j}\right)(x)\right) d x\right)_{j, k=0}^{N-1} .
\end{gathered}
$$

If $\left(M^{(1)}\right)^{-1}=\left(\mu_{j k}\right)_{j, k=0}^{N-1}$, then

$$
\left[G_{N}^{(1)}(f)\right]^{2}=\operatorname{det}\left(I+K_{N}^{(1)} f\right)
$$

where $K_{N}^{(1)}$ is an integral operator

$$
K_{N}^{(1)}=\left(\begin{array}{cc}
-\sum_{j, k=0}^{N-1} \mu_{j k} \psi_{j} \otimes \varepsilon \psi_{k} & \sum_{j, k=0}^{N-1} \mu_{j k} \psi_{j} \otimes \psi_{k} \\
-\sum_{j, k=0}^{N-1} \mu_{j k} \varepsilon \psi_{j} \otimes \varepsilon \psi_{k}-\varepsilon & \sum_{j, k=0}^{N-1} \mu_{j k} \varepsilon \psi_{j} \otimes \psi_{k}
\end{array}\right)=:\left(\begin{array}{cc}
K_{N, 1}^{(1,1)} & K_{N, 1}^{(1,2)} \\
K_{N, 1}^{(2,1)} & K_{N, 1}^{(2,2)}
\end{array}\right) .
$$

In the next theorem, we obtain relations on $K_{N, 1}^{(i, j)}, i, j=1,2$.

\section{Theorem 4.1.}

$$
K_{N, 1}^{(1,2)}=D K_{N, 1}^{(2,2)}, \quad K_{N, 1}^{(2,1)}=K_{N, 1}^{(2,2)} \varepsilon-\varepsilon, \quad K_{N, 1}^{(1,1)}=D K_{N, 1}^{(2,2)} \varepsilon .
$$


Proof. For any integrable function $g(x)$ supported on $[a, b]$, we have

$$
\begin{aligned}
D K_{N, 1}^{(2,2)} g(x) & =\frac{d}{d x} \int_{a}^{b} K_{N, 1}^{(2,2)}(x, y) g(y) d y \\
& =\int_{a}^{b} \frac{\partial}{\partial x} K_{N, 1}^{(2,2)}(x, y) g(y) d y \\
& =\int_{a}^{b} \frac{\partial}{\partial x} \sum_{j, k=0}^{N-1} \varepsilon \psi_{j}(x) \mu_{j k} \psi_{k}(y) g(y) d y \\
& =\int_{a}^{b} \sum_{j, k=0}^{N-1}\left(D \varepsilon \psi_{j}(x)\right) \mu_{j k} \psi_{k}(y) g(y) d y \\
& =\int_{a}^{b} \sum_{j, k=0}^{N-1} \psi_{j}(x) \mu_{j k} \psi_{k}(y) g(y) d y \\
& =\int_{a}^{b} K_{N, 1}^{(1,2)}(x, y) g(y) d y \\
& =K_{N, 1}^{(1,2)} g(x),
\end{aligned}
$$

which implies,

$$
K_{N, 1}^{(1,2)}=D K_{N, 1}^{(2,2)}
$$

Note that

$$
\begin{aligned}
K_{N, 1}^{(2,2)} \varepsilon & =\sum_{j, k=0}^{N-1} \mu_{j k} \varepsilon \psi_{j} \otimes \psi_{k} \varepsilon \\
& =-\sum_{j, k=0}^{N-1} \mu_{j k} \varepsilon \psi_{j} \otimes \varepsilon \psi_{k} \\
& =K_{N, 1}^{(2,1)}+\varepsilon
\end{aligned}
$$

that is,

$$
K_{N, 1}^{(2,1)}=K_{N, 1}^{(2,2)} \varepsilon-\varepsilon
$$

Moreover, from (4.2),

$$
\begin{aligned}
D K_{N, 1}^{(2,2)} \varepsilon & =-\sum_{j, k=0}^{N-1} \mu_{j k}\left(D \varepsilon \psi_{j}\right) \otimes \varepsilon \psi_{k} \\
& =-\sum_{j, k=0}^{N-1} \mu_{j k} \psi_{j} \otimes \varepsilon \psi_{k} \\
& =K_{N, 1}^{(1,1)}
\end{aligned}
$$

The proof is complete. 
The series of computations presented below takes the determinant into the form of identity plus scalar operators. From Theorem 4.1, $K_{N}^{(1)}$ can be written as

$$
\begin{aligned}
K_{N}^{(1)} & =\left(\begin{array}{cc}
D K_{N, 1}^{(2,2)} \varepsilon & D K_{N, 1}^{(2,2)} \\
K_{N, 1}^{(2,2)} \varepsilon-\varepsilon & K_{N, 1}^{(2,2)}
\end{array}\right) \\
& =\left(\begin{array}{cc}
D & 0 \\
0 & I
\end{array}\right)\left(\begin{array}{cc}
K_{N, 1}^{(2,2)} \varepsilon & K_{N, 1}^{(2,2)} \\
K_{N, 1}^{(2,2)} \varepsilon-\varepsilon & K_{N, 1}^{(2,2)}
\end{array}\right) \\
& =: \widetilde{A} \widetilde{B}
\end{aligned}
$$

where

$$
\widetilde{A}:=\left(\begin{array}{cc}
D & 0 \\
0 & I
\end{array}\right), \quad \widetilde{B}:=\left(\begin{array}{cc}
K_{N, 1}^{(2,2)} \varepsilon & K_{N, 1}^{(2,2)} \\
K_{N, 1}^{(2,2)} \varepsilon-\varepsilon & K_{N, 1}^{(2,2)}
\end{array}\right) .
$$

By Theorem 2.6, we have

$$
\left[G_{N}^{(1)}(f)\right]^{2}=\operatorname{det}(I+\widetilde{A} \widetilde{B} f)=\operatorname{det}(I+\widetilde{B} f \widetilde{A}) .
$$

Since

$$
\begin{aligned}
\widetilde{B} f \widetilde{A} & =\left(\begin{array}{cc}
K_{N, 1}^{(2,2)} \varepsilon & K_{N, 1}^{(2,2)} \\
K_{N, 1}^{(2,2)} \varepsilon-\varepsilon & K_{N, 1}^{(2,2)}
\end{array}\right) f\left(\begin{array}{cc}
D & 0 \\
0 & I
\end{array}\right) \\
& =\left(\begin{array}{cc}
K_{N, 1}^{(2,2)} \varepsilon & K_{N, 1}^{(2,2)} \\
K_{N, 1}^{(2,2)} \varepsilon-\varepsilon & K_{N, 1}^{(2,2)}
\end{array}\right)\left(\begin{array}{cc}
f D & 0 \\
0 & f
\end{array}\right) \\
& =\left(\begin{array}{cc}
K_{N, 1}^{(2,2)} \varepsilon f D & K_{N, 1}^{(2,2)} f \\
K_{N, 1}^{(2,2)} \varepsilon f D-\varepsilon f D & K_{N, 1}^{(2,2)} f
\end{array}\right),
\end{aligned}
$$

then

$$
\left[G_{N}^{(1)}(f)\right]^{2}=\operatorname{det}\left(\begin{array}{cc}
I+K_{N, 1}^{(2,2)} \varepsilon f D & K_{N, 1}^{(2,2)} f \\
K_{N, 1}^{(2,2)} \varepsilon f D-\varepsilon f D & I+K_{N, 1}^{(2,2)} f
\end{array}\right) .
$$

The computation below reduces the above into a determinant of scalar operators. We subtract row 1 from row 2 ,

$$
\left[G_{N}^{(1)}(f)\right]^{2}=\operatorname{det}\left(\begin{array}{cc}
I+K_{N, 1}^{(2,2)} \varepsilon f D & K_{N, 1}^{(2,2)} f \\
-I-\varepsilon f D & I
\end{array}\right) .
$$


Next, add column 2 times $I+\varepsilon f D$ to column 1 ,

$$
\begin{aligned}
{\left[G_{N}^{(1)}(f)\right]^{2} } & =\operatorname{det}\left(\begin{array}{cc}
I+K_{N, 1}^{(2,2)} \varepsilon f D+K_{N, 1}^{(2,2)} f(I+\varepsilon f D) & K_{N, 1}^{(2,2)} f \\
0 & I
\end{array}\right) \\
& =\operatorname{det}\left(I+K_{N, 1}^{(2,2)}(\varepsilon f D+f+f \varepsilon f D)\right) .
\end{aligned}
$$

Remark. The above result agrees with [6] for the GOE case if we take $f=-\mu \chi_{J}$, where $\chi_{J}$ is the characteristic function of the interval $J$.

Using (3.3), Lemma 2.7 and note that $f$ is a Schwartz function, then we have the following theorem.

\section{Theorem 4.2.}

$$
\left[G_{N}^{(1)}(f)\right]^{2}=\operatorname{det}\left(I+K_{N, 1}^{(2,2)}\left(f^{2}+2 f\right)-K_{N, 1}^{(2,2)} \varepsilon f^{\prime}-K_{N, 1}^{(2,2)} f \varepsilon f^{\prime}\right)
$$

where the kernel of $K_{N, 1}^{(2,2)}$ reads

$$
K_{N, 1}^{(2,2)}(x, y)=\sum_{j, k=0}^{N-1} \mu_{j k} \varepsilon \psi_{j}(x) \psi_{k}(y)
$$

\subsection{GOE}

It is convenient in this case to choose

$$
w(x)=\mathrm{e}^{-\frac{x^{2}}{2}}, \quad x \in \mathbb{R}
$$

following Dieng and Tracy-Widom's discussion [6, 15]. We want to choose $\psi_{j}$ to make $M^{(1)}$ simplest possible. Define

$$
\psi_{2 n+1}(x):=\frac{d}{d x} \varphi_{2 n}(x), \quad \psi_{2 n}(x):=\varphi_{2 n}(x), \quad n=0,1,2, \ldots
$$

where $\varphi_{j}(x)$ is given by (3.5),

$$
\varphi_{j}(x)=\frac{H_{j}(x)}{c_{j}} \mathrm{e}^{-\frac{x^{2}}{2}}, \quad c_{j}=\pi^{\frac{1}{4}} 2^{\frac{j}{2}} \sqrt{\Gamma(j+1)} .
$$

We can check that this definition satisfies (4.1), i.e., $\psi_{j}(x)=\pi_{j}(x) \mathrm{e}^{-\frac{x^{2}}{2}}, j=0,1,2, \ldots$, where $\pi_{j}(x)$ is a polynomial of degree $j$. The matrix $M^{(1)}:=\left(\int_{-\infty}^{\infty} \psi_{j}(x) \varepsilon \psi_{k}(x) d x\right)_{j, k=0}^{N-1}$ is computed below from (4.3). 


\section{Lemma 4.3. (Dieng, Tracy - Widom)}

$$
M^{(1)}=\left(\begin{array}{ccccccc}
0 & 1 & 0 & 0 & \cdots & 0 & 0 \\
-1 & 0 & 0 & 0 & \cdots & 0 & 0 \\
0 & 0 & 0 & 1 & \cdots & 0 & 0 \\
0 & 0 & -1 & 0 & \cdots & 0 & 0 \\
\vdots & \vdots & \vdots & \vdots & & \vdots & \vdots \\
0 & 0 & 0 & 0 & \cdots & 0 & 1 \\
0 & 0 & 0 & 0 & \cdots & -1 & 0
\end{array}\right)_{N \times N} .
$$

It's obvious that $\left(M^{(1)}\right)^{-1}=-M^{(1)}$, so $\mu_{2 j, 2 j+1}=-1, \mu_{2 j+1,2 j}=1$, and $\mu_{j k}=0$ for other cases, hence

$$
\begin{aligned}
K_{N, 1}^{(2,2)}(x, y) & =\sum_{j, k=0}^{N-1} \mu_{j k} \varepsilon \psi_{j}(x) \psi_{k}(y) \\
& =-\sum_{j=0}^{\frac{N}{2}-1} \varepsilon \psi_{2 j}(x) \psi_{2 j+1}(y)+\sum_{j=0}^{\frac{N}{2}-1} \varepsilon \psi_{2 j+1}(x) \psi_{2 j}(y) \\
& =\sum_{j=0}^{\frac{N}{2}-1} \varphi_{2 j}(x) \varphi_{2 j}(y)-\sum_{j=0}^{\frac{N}{2}-1} \varepsilon \varphi_{2 j}(x) \varphi_{2 j}^{\prime}(y)
\end{aligned}
$$

By (3.11), we have

$$
\begin{aligned}
\sum_{j=0}^{\frac{N}{2}-1} \varepsilon \varphi_{2 j}(x) \varphi_{2 j}^{\prime}(y) & =\sum_{j=0}^{\frac{N}{2}-1} \varepsilon \varphi_{2 j}(x)\left(\sqrt{j} \varphi_{2 j-1}(y)-\sqrt{j+\frac{1}{2}} \varphi_{2 j+1}(y)\right) \\
& =\sum_{j=0}^{\frac{N}{2}-1} \sqrt{j} \varepsilon \varphi_{2 j}(x) \varphi_{2 j-1}(y)-\sum_{j=0}^{\frac{N}{2}-1} \sqrt{j+\frac{1}{2}} \varepsilon \varphi_{2 j}(x) \varphi_{2 j+1}(y) \\
& =\sum_{j=1}^{\frac{N}{2}-1} \sqrt{j} \varepsilon \varphi_{2 j}(x) \varphi_{2 j-1}(y)-\sum_{j=1}^{\frac{N}{2}} \sqrt{j-\frac{1}{2}} \varepsilon \varphi_{2 j-2}(x) \varphi_{2 j-1}(y) \\
& =\sum_{j=1}^{\frac{N}{2}} \sqrt{j} \varepsilon \varphi_{2 j}(x) \varphi_{2 j-1}(y)-\sqrt{\frac{N}{2}} \varepsilon \varphi_{N}(x) \varphi_{N-1}(y)-\sum_{j=1}^{\frac{N}{2}} \sqrt{j-\frac{1}{2}} \varepsilon \varphi_{2 j-2}(x) \varphi_{2 j-1}(y) \\
& =-\sum_{j=1}^{\frac{N}{2}}\left(\sqrt{j-\frac{1}{2}} \varepsilon \varphi_{2 j-2}(x)-\sqrt{j} \varepsilon \varphi_{2 j}(x)\right) \varphi_{2 j-1}(y)-\sqrt{\frac{N}{2}} \varepsilon \varphi_{N}(x) \varphi_{N-1}(y)
\end{aligned}
$$

Using (3.11) again, we find

$$
\varphi_{2 j-1}^{\prime}(x)=\sqrt{j-\frac{1}{2}} \varphi_{2 j-2}(x)-\sqrt{j} \varphi_{2 j}(x),
$$


then according to Lemma 2.7 ,

$$
\varphi_{2 j-1}(x)=\varepsilon \varphi_{2 j-1}^{\prime}(x)=\sqrt{j-\frac{1}{2}} \varepsilon \varphi_{2 j-2}(x)-\sqrt{j} \varepsilon \varphi_{2 j}(x) .
$$

Hence

$$
\sum_{j=0}^{\frac{N}{2}-1} \varepsilon \varphi_{2 j}(x) \varphi_{2 j}^{\prime}(y)=-\sum_{j=1}^{\frac{N}{2}} \varphi_{2 j-1}(x) \varphi_{2 j-1}(y)-\sqrt{\frac{N}{2}} \varepsilon \varphi_{N}(x) \varphi_{N-1}(y) .
$$

It follows that

$$
\begin{aligned}
K_{N, 1}^{(2,2)}(x, y) & =\sum_{j=0}^{\frac{N}{2}-1} \varphi_{2 j}(x) \varphi_{2 j}(y)+\sum_{j=1}^{\frac{N}{2}} \varphi_{2 j-1}(x) \varphi_{2 j-1}(y)+\sqrt{\frac{N}{2}} \varepsilon \varphi_{N}(x) \varphi_{N-1}(y) \\
& =\sum_{j=0}^{N-1} \varphi_{j}(x) \varphi_{j}(y)+\sqrt{\frac{N}{2}} \varepsilon \varphi_{N}(x) \varphi_{N-1}(y) \\
& =S_{N}(x, y)+\sqrt{\frac{N}{2}} \varepsilon \varphi_{N}(x) \varphi_{N-1}(y)
\end{aligned}
$$

where

$$
S_{N}(x, y):=\sum_{j=0}^{N-1} \varphi_{j}(x) \varphi_{j}(y)=\sqrt{\frac{N}{2}} \frac{\varphi_{N}(x) \varphi_{N-1}(y)-\varphi_{N}(y) \varphi_{N-1}(x)}{x-y} .
$$

Here we have used the Christoffel-Darboux formula in the last equality. Note that $S_{N}(x, y)=K_{N}^{(2)}(x, y)$.

By Theorem 4.2, we have the following theorem.

\section{Theorem 4.4.}

$$
\begin{aligned}
{\left[G_{N}^{(1)}(f)\right]^{2} } & =\operatorname{det}\left(I+S_{N}\left(f^{2}+2 f\right)-S_{N} \varepsilon f^{\prime}-S_{N} f \varepsilon f^{\prime}+\sqrt{\frac{N}{2}} \varepsilon \varphi_{N} \otimes \varphi_{N-1}\left(f^{2}+2 f\right)\right. \\
& \left.+\sqrt{\frac{N}{2}}\left(\varepsilon \varphi_{N} \otimes \varepsilon \varphi_{N-1}\right) f^{\prime}+\sqrt{\frac{N}{2}}\left(\varepsilon \varphi_{N} \otimes \varepsilon\left(\varphi_{N-1} f\right)\right) f^{\prime}\right) .
\end{aligned}
$$

\subsection{Large $N$ behavior of the GOE moment generating function}

Now we consider the scaling limit of $\left[G_{N}^{(1)}(f)\right]^{2}$. Write

$$
\left[G_{N}^{(1)}(f)\right]^{2}=: \operatorname{det}(I+T)
$$

where

$$
\begin{aligned}
T: & =S_{N}\left(f^{2}+2 f\right)-S_{N} \varepsilon f^{\prime}-S_{N} f \varepsilon f^{\prime}+\sqrt{\frac{N}{2}} \varepsilon \varphi_{N} \otimes \varphi_{N-1}\left(f^{2}+2 f\right) \\
& +\sqrt{\frac{N}{2}}\left(\varepsilon \varphi_{N} \otimes \varepsilon \varphi_{N-1}\right) f^{\prime}+\sqrt{\frac{N}{2}}\left(\varepsilon \varphi_{N} \otimes \varepsilon\left(\varphi_{N-1} f\right)\right) f^{\prime} .
\end{aligned}
$$


In the computations below, we replace $f(x)$ by $f(\sqrt{2 N} x)$ and $f^{\prime}(x)$ by

$$
f^{\prime}(\sqrt{2 N} x)=\frac{d}{\sqrt{2 N} d x} f(\sqrt{2 N} x) .
$$

Similarly to the GSE case, we have the following theorems.

\section{Theorem 4.5.}

$$
\begin{gathered}
\lim _{N \rightarrow \infty} \frac{1}{\sqrt{2 N}} S_{N}\left(\frac{x}{\sqrt{2 N}}, \frac{y}{\sqrt{2 N}}\right)=\frac{\sin (x-y)}{\pi(x-y)}, \\
\lim _{N \rightarrow \infty} \frac{1}{\sqrt{2 N}} S_{N}\left(\frac{x}{\sqrt{2 N}}, \frac{x}{\sqrt{2 N}}\right)=\frac{1}{\pi} .
\end{gathered}
$$

\section{Theorem 4.6.}

$$
\begin{gathered}
\varphi_{N-1}\left(\frac{x}{\sqrt{2 N}}\right)=-(-1)^{\frac{N}{2}} 2^{\frac{1}{4}} \pi^{-\frac{1}{2}} N^{-\frac{1}{4}} \sin x+O\left(N^{-\frac{3}{4}}\right), N \rightarrow \infty \\
\varepsilon \varphi_{N}\left(\frac{x}{\sqrt{2 N}}\right)=(-1)^{\frac{N}{2}} 2^{-\frac{1}{4}} \pi^{-\frac{1}{2}} N^{-\frac{3}{4}} \sin x+O\left(N^{-\frac{5}{4}}\right), N \rightarrow \infty \\
\varepsilon \varphi_{N-1}\left(\frac{x}{\sqrt{2 N}}\right)=-2^{-\frac{1}{4}} N^{-\frac{1}{4}}+O\left(N^{-\frac{3}{4}}\right), N \rightarrow \infty, \\
\varepsilon\left(\varphi_{N-1} f\right)\left(\frac{x}{\sqrt{2 N}}\right)=-(-1)^{\frac{N}{2}} 2^{-\frac{5}{4}} \pi^{-\frac{1}{2}} N^{-\frac{3}{4}}\left[\int_{-\infty}^{x} \sin y f(y) d y-\int_{x}^{\infty} \sin y f(y) d y\right]+O\left(N^{-\frac{5}{4}}\right), \quad N \rightarrow \infty .
\end{gathered}
$$

Using Theorem 4.5 and Theorem 4.6 to compute $\operatorname{Tr} T$ and $\operatorname{Tr} T^{2}$ as $N \rightarrow \infty$. we have

$$
\begin{aligned}
\operatorname{Tr} T & =\frac{1}{\pi} \int_{-\infty}^{\infty}\left[f^{2}(x)+2 f(x)\right] d x \\
& -\frac{1}{2 \pi \sqrt{2 N}} \int_{-\infty}^{\infty}\left[\int_{x}^{\infty} \frac{\sin (x-y)}{x-y} f(y) d y-\int_{-\infty}^{x} \frac{\sin (x-y)}{x-y} f(y) d y\right] f^{\prime}(x) d x \\
& -\frac{1}{2 \pi N} \int_{-\infty}^{\infty} \sin ^{2} x\left[f^{2}(x)+2 f(x)\right] d x-\frac{(-1)^{\frac{N}{2}}}{2 \sqrt{2 \pi} N} \int_{-\infty}^{\infty} \sin x f^{\prime}(x) d x+O\left(N^{-\frac{3}{2}}\right), N \rightarrow \infty
\end{aligned}
$$

and

$$
\begin{aligned}
\operatorname{Tr} T^{2}= & \frac{1}{\pi^{2}} \int_{-\infty}^{\infty} \int_{-\infty}^{\infty}\left[\frac{\sin (x-y)}{x-y}\right]^{2}\left[f^{2}(x)+2 f(x)\right]\left[f^{2}(y)+2 f(y)\right] d x d y \\
+ & \frac{2}{\pi^{2} \sqrt{2 N}} \int_{-\infty}^{\infty} \int_{-\infty}^{\infty} \frac{\sin (x-y)}{x-y} \operatorname{Si}(x-y) f^{\prime}(x)\left[f^{2}(y)+2 f(y)\right] d x d y \\
- & \frac{1}{\pi^{2} \sqrt{2 N}} \int_{-\infty}^{\infty} \int_{-\infty}^{\infty}\left[\int_{x}^{\infty} \frac{\sin (y-z)}{y-z} f(z) d z-\int_{-\infty}^{x} \frac{\sin (y-z)}{y-z} f(z) d z\right] \frac{\sin (x-y)}{x-y} \\
& f^{\prime}(x)\left[f^{2}(y)+2 f(y)\right] d x d y+O\left(N^{-1}\right), \quad N \rightarrow \infty .
\end{aligned}
$$


Now we want to find the mean and variance of the linear statistics $\sum_{j=1}^{N} F\left(\sqrt{2 N} x_{j}\right)$, since

$$
f(\sqrt{2 N} x) \approx-\lambda F(\sqrt{2 N} x)+\frac{\lambda^{2}}{2} F^{2}(\sqrt{2 N} x)
$$

we replace $f$ with $-\lambda F+\frac{\lambda^{2}}{2} F^{2}$ in the expression of $\operatorname{Tr} T$ and $\operatorname{Tr} T^{2}$, we have

$$
\begin{aligned}
& \log \operatorname{det}(I+T) \\
= & -\lambda\left\{\frac{2}{\pi} \int_{-\infty}^{\infty} F(x) d x-\frac{1}{\pi N} \int_{-\infty}^{\infty} \sin ^{2} x F(x) d x-\frac{(-1)^{\frac{N}{2}}}{2 \sqrt{2 \pi} N} \int_{-\infty}^{\infty} \sin x F^{\prime}(x) d x+O\left(N^{-\frac{3}{2}}\right)\right\} \\
+ & \frac{\lambda^{2}}{2}\left\{\frac{4}{\pi} \int_{-\infty}^{\infty} F^{2}(x) d x-\frac{4}{\pi^{2}} \int_{-\infty}^{\infty} \int_{-\infty}^{\infty}\left[\frac{\sin (x-y)}{x-y}\right]^{2} F(x) F(y) d x d y\right. \\
- & \frac{1}{\pi \sqrt{2 N}} \int_{-\infty}^{\infty}\left[\int_{x}^{\infty} \frac{\sin (x-y)}{x-y} F(y) d y-\int_{-\infty}^{x} \frac{\sin (x-y)}{x-y} F(y) d y\right] F^{\prime}(x) d x \\
- & \left.\frac{4}{\pi^{2} \sqrt{2 N}} \int_{-\infty}^{\infty} \int_{-\infty}^{\infty} \frac{\sin (x-y)}{x-y} \operatorname{Si}(x-y) F^{\prime}(x) F(y) d x d y+O\left(N^{-1}\right)\right\}, N \rightarrow \infty
\end{aligned}
$$

Denote by $\mu_{N}^{(G O E)}$ and $\mathcal{V}_{N}^{(G O E)}$ the mean and variance of the linear statistics $\sum_{j=1}^{N} F\left(\sqrt{2 N} x_{j}\right)$, then we have the following theorem.

Theorem 4.7. As $N \rightarrow \infty$,

$$
\begin{aligned}
\mu_{N}^{(G O E)} & =\mu_{N}^{(G U E)}-\frac{1}{2 \pi N} \int_{-\infty}^{\infty} \sin ^{2} x F(x) d x-\frac{(-1)^{\frac{N}{2}}}{4 \sqrt{2 \pi} N} \int_{-\infty}^{\infty} \sin x F^{\prime}(x) d x+O\left(N^{-\frac{3}{2}}\right), \\
\mathcal{V}_{N}^{(G O E)}= & 2 \mathcal{V}_{N}^{(G U E)}-\frac{1}{2 \pi \sqrt{2 N}} \int_{-\infty}^{\infty}\left[\int_{x}^{\infty} \frac{\sin (x-y)}{x-y} F(y) d y-\int_{-\infty}^{x} \frac{\sin (x-y)}{x-y} F(y) d y\right] F^{\prime}(x) d x \\
- & \frac{2}{\pi^{2} \sqrt{2 N}} \int_{-\infty}^{\infty} \int_{-\infty}^{\infty} \frac{\sin (x-y)}{x-y} \operatorname{Si}(x-y) F^{\prime}(x) F(y) d x d y+O\left(N^{-1}\right)
\end{aligned}
$$

where $\mu_{N}^{(G U E)}$ and $\mathcal{V}_{N}^{(G U E)}$ for $N \rightarrow \infty$ are given in (1.2) and (1.3) respectively.

\subsection{LOE}

We now specialize results obtained in Section 4.1 to the situation where $w$ is taken to be the square root of the Laguerre weight, namely,

$$
w(x)=x^{\frac{\alpha}{2}} \mathrm{e}^{-\frac{x}{2}}, \quad \alpha>-2, x \in \mathbb{R}^{+} .
$$

Again we choose a special $\psi_{j}$ so that $M^{(1)}$ is as simple as possible. Let

$$
\psi_{2 n+1}(x):=\frac{d}{d x} \varphi_{2 n}^{(\alpha+1)}(x), \quad \psi_{2 n}(x):=\widetilde{\varphi}_{2 n}^{(\alpha+1)}(x), \quad n=0,1,2, \ldots,
$$


where $\varphi_{j}^{(\alpha+1)}(x)$ and $\widetilde{\varphi}_{j}^{(\alpha+1)}(x)$ are given by

$$
\begin{gathered}
\varphi_{j}^{(\alpha+1)}(x):=\frac{L_{j}^{(\alpha+1)}(x)}{c_{j}^{(\alpha+1)}} x^{\frac{\alpha}{2}+1} \mathrm{e}^{-\frac{x}{2}}, \quad j=0,1,2, \ldots \\
\widetilde{\varphi}_{j}^{(\alpha+1)}(x):=\frac{L_{j}^{(\alpha+1)}(x)}{c_{j}^{(\alpha+1)}} x^{\frac{\alpha}{2}} \mathrm{e}^{-\frac{x}{2}}, \quad j=0,1,2, \ldots
\end{gathered}
$$

It's easy to see that

$$
\int_{0}^{\infty} \varphi_{j}^{(\alpha+1)}(x) \widetilde{\varphi}_{k}^{(\alpha+1)}(x) d x=\delta_{j k}, \quad j, k=0,1,2, \ldots
$$

The next theorem shows that (4.4) satisfies (4.1), namely, $\psi_{j}(x) x^{-\frac{\alpha}{2}} \mathrm{e}^{\frac{x}{2}}$ is a polynomial of degree $j$.

Theorem 4.8. $\psi_{j}(x) x^{-\frac{\alpha}{2}} \mathrm{e}^{\frac{x}{2}}, j=0,1,2, \ldots$ is a polynomial of degree $j$.

Proof. We prove this by considering two cases, $j$ even and $j$ odd. It is clear for even $j$, that $\psi_{2 j} x^{-\alpha / 2} \mathrm{e}^{x / 2}$ is up to a constant multiple of $L_{2 j}^{(\alpha+1)}(x)$.

If $j$ is odd, then we find,

$$
\begin{aligned}
\psi_{2 n+1}(x) x^{-\frac{\alpha}{2}} \mathrm{e}^{\frac{x}{2}} & =\left[\varphi_{2 n}^{(\alpha+1)}(x)\right]^{\prime} x^{-\frac{\alpha}{2}} \mathrm{e}^{\frac{x}{2}} \\
& =\left(\frac{1}{c_{2 n}^{(\alpha+1)}} L_{2 n}^{(\alpha+1)}(x) x^{\frac{\alpha}{2}+1} \mathrm{e}^{-\frac{x}{2}}\right)^{\prime} x^{-\frac{\alpha}{2}} \mathrm{e}^{\frac{x}{2}} \\
& =\frac{1}{c_{2 n}^{(\alpha+1)}}\left[x\left(L_{2 n}^{(\alpha+1)}(x)\right)^{\prime}+\left(\frac{\alpha}{2}+1\right) L_{2 n}^{(\alpha+1)}(x)-\frac{1}{2} x L_{2 n}^{(\alpha+1)}(x)\right], \quad n=0,1,2, \ldots
\end{aligned}
$$

Thus $\psi_{2 n+1}(x) x^{-\alpha / 2} \mathrm{e}^{\frac{x^{2}}{2}}$ is a polynomial of degree $2 n+1$. The proof is complete.

We use (4.4) to compute $M^{(1)}:=\left(\int_{0}^{\infty} \psi_{j}(x) \varepsilon \psi_{k}(x) d x\right)_{j, k=0}^{N-1}$, resulting in the following theorem.

\section{Theorem 4.9.}

$$
M^{(1)}=\left(\begin{array}{ccccccc}
0 & 1 & 0 & 0 & \cdots & 0 & 0 \\
-1 & 0 & 0 & 0 & \cdots & 0 & 0 \\
0 & 0 & 0 & 1 & \cdots & 0 & 0 \\
0 & 0 & -1 & 0 & \cdots & 0 & 0 \\
\vdots & \vdots & \vdots & \vdots & & \vdots & \vdots \\
0 & 0 & 0 & 0 & \cdots & 0 & 1 \\
0 & 0 & 0 & 0 & \cdots & -1 & 0
\end{array}\right)_{N \times N}
$$


Proof. Let $m_{j k}$ be the $(j, k)$-entry of $M^{(1)}$, again separating into four cases: $(j, k)=($ even, odd), (odd, even), (even, even) and (odd, odd). We find, for the (even, odd) case,

$$
\begin{aligned}
m_{2 j, 2 k+1} & =\int_{0}^{\infty} \psi_{2 j}(x) \varepsilon \psi_{2 k+1}(x) d x \\
& =\int_{0}^{\infty} \widetilde{\varphi}_{2 j}^{(\alpha+1)}(x)\left(\varepsilon D \varphi_{2 k}^{(\alpha+1)}(x)\right) d x \\
& =\int_{0}^{\infty} \widetilde{\varphi}_{2 j}^{(\alpha+1)}(x) \varphi_{2 k}^{(\alpha+1)}(x) d x \\
& =\delta_{j k} .
\end{aligned}
$$

For the (odd, even) case, note that $M^{(1)}$ is antisymmetric, then

$$
\begin{aligned}
m_{2 j+1,2 k} & =-m_{2 k, 2 j+1} \\
& =-\delta_{j k} .
\end{aligned}
$$

The (even, even) case,

$$
\begin{aligned}
m_{2 j, 2 k} & =\int_{0}^{\infty} \psi_{2 j}(x) \varepsilon \psi_{2 k}(x) d x \\
& =\int_{0}^{\infty} \widetilde{\varphi}_{2 j}^{(\alpha+1)}(x) \varepsilon \widetilde{\varphi}_{2 k}^{(\alpha+1)}(x) d x \\
& =\frac{1}{2 c_{2 j}^{(\alpha+1)} c_{2 k}^{(\alpha+1)}} \int_{0}^{\infty} L_{2 j}^{(\alpha+1)}(x) x^{\frac{\alpha}{2}} \mathrm{e}^{-\frac{x}{2}}\left(\int_{0}^{x} L_{2 k}^{(\alpha+1)}(y) y^{\frac{\alpha}{2}} \mathrm{e}^{-\frac{y}{2}} d y-\int_{x}^{\infty} L_{2 k}^{(\alpha+1)}(y) y^{\frac{\alpha}{2}} \mathrm{e}^{-\frac{y}{2}} d y\right) d x \\
& =\frac{1}{2 c_{2 j}^{(\alpha+1)} c_{2 k}^{(\alpha+1)}} \int_{0}^{\infty} L_{2 j}^{(\alpha+1)}(x) x^{\frac{\alpha}{2}} \mathrm{e}^{-\frac{x}{2}}\left(2 \int_{0}^{x} L_{2 k}^{(\alpha+1)}(y) y^{\frac{\alpha}{2}} \mathrm{e}^{-\frac{y}{2}} d y-\int_{0}^{\infty} L_{2 k}^{(\alpha+1)}(y) y^{\frac{\alpha}{2}} \mathrm{e}^{-\frac{y}{2}} d y\right) d x \\
& =\frac{1}{2 c_{2 j}^{(\alpha+1)} c_{2 k}^{(\alpha+1)}}\left(2 \int_{0}^{\infty} L_{2 j}^{(\alpha+1)}(x) x^{\frac{\alpha}{2}} \mathrm{e}^{-\frac{x}{2}} d x \int_{0}^{x} L_{2 k}^{(\alpha+1)}(y) y^{\frac{\alpha}{2}} \mathrm{e}^{-\frac{y}{2}} d y\right. \\
& \left.-\int_{0}^{\infty} L_{2 j}^{(\alpha+1)}(x) x^{\frac{\alpha}{2}} \mathrm{e}^{-\frac{x}{2}} d x \int_{0}^{\infty} L_{2 k}^{(\alpha+1)}(y) y^{\frac{\alpha}{2}} \mathrm{e}^{-\frac{y}{2}} d y\right) \\
& =\frac{1}{2 c_{2 j}^{(\alpha+1)} c_{2 k}^{(\alpha+1)}}\left[\frac{2^{\alpha+2} \Gamma\left(j+1+\frac{\alpha}{2}\right) \Gamma\left(k+1+\frac{\alpha}{2}\right)}{j ! k !}-\frac{2^{\alpha+2} \Gamma\left(j+1+\frac{\alpha}{2}\right) \Gamma\left(k+1+\frac{\alpha}{2}\right)}{j ! k !}\right] \\
& =0, \quad(k)
\end{aligned}
$$

where we have used the fact

$$
\int_{0}^{\infty} L_{2 j}^{(\alpha+1)}(x) x^{\frac{\alpha}{2}} \mathrm{e}^{-\frac{x}{2}} d x=\frac{2^{\frac{\alpha}{2}+1} \Gamma\left(j+1+\frac{\alpha}{2}\right)}{j !}, j=0,1,2, \ldots
$$


Finally, the (odd, odd) case. If $j<k$,

$$
\begin{aligned}
m_{2 j+1,2 k+1} & =\int_{0}^{\infty} \psi_{2 j+1}(x) \varepsilon \psi_{2 k+1}(x) d x \\
& =\int_{0}^{\infty}\left[\varphi_{2 j}^{(\alpha+1)}(x)\right]^{\prime} \varepsilon D \varphi_{2 k}^{(\alpha+1)}(x) d x \\
& =\int_{0}^{\infty}\left[\varphi_{2 j}^{(\alpha+1)}(x)\right]^{\prime} \varphi_{2 k}^{(\alpha+1)}(x) d x \\
& =\int_{0}^{\infty} \frac{1}{c_{2 j}^{(\alpha+1)}}\left[x\left(L_{2 j}^{(\alpha+1)}(x)\right)^{\prime}+\left(\frac{\alpha}{2}+1\right) L_{2 j}^{(\alpha+1)}(x)-\frac{1}{2} x L_{2 j}^{(\alpha+1)}(x)\right] x^{\frac{\alpha}{2}} \mathrm{e}^{-\frac{x}{2}} \\
& \frac{1}{c_{2 k}^{(\alpha+1)}} L_{2 k}^{(\alpha+1)}(x) x^{\frac{\alpha}{2}+1} \mathrm{e}^{-\frac{x}{2}} d x \\
= & \frac{1}{c_{2 j}^{(\alpha+1)} c_{2 k}^{(\alpha+1)}} \int_{0}^{\infty} L_{2 k}^{(\alpha+1)}(x)\left[x\left(L_{2 j}^{(\alpha+1)}(x)\right)^{\prime}+\left(\frac{\alpha}{2}+1\right) L_{2 j}^{(\alpha+1)}(x)-\frac{1}{2} x L_{2 j}^{(\alpha+1)}(x)\right] x^{\alpha+1} \mathrm{e}^{-x} d x \\
= & 0,
\end{aligned}
$$

since, $2 j+1$, the degree of the polynomials $x\left(L_{2 j}^{(\alpha+1)}(x)\right)^{\prime}+\left(\frac{\alpha}{2}+1\right) L_{2 j}^{(\alpha+1)}(x)-\frac{1}{2} x L_{2 j}^{(\alpha+1)}(x)$, is less than $2 k$.

If $j>k$, due to the fact that $M^{(1)}$ is antisymmetric,

$$
\begin{aligned}
m_{2 j+1,2 k+1} & =-m_{2 k+1,2 j+1} \\
& =0
\end{aligned}
$$

hence

$$
m_{2 j+1,2 k+1}=0, \quad j, k=0,1, \ldots
$$

It is the desired result for $M^{(1)}$. The proof is complete.

We begin here a series of computations analogues to those in derivation of the LSE problem, ending up with an expression for $\left[G_{N}^{(1)}(f)\right]^{2}$ as a scalar Fredholm determinant.

It's obvious that $\left(M^{(1)}\right)^{-1}=-M^{(1)}$, so $\mu_{2 j, 2 j+1}=-1, \mu_{2 j+1,2 j}=1$, and $\mu_{j k}=0$ for other cases, hence

$$
\begin{aligned}
K_{N, 1}^{(2,2)}(x, y) & =\sum_{j, k=0}^{N-1} \mu_{j k} \varepsilon \psi_{j}(x) \psi_{k}(y) \\
& =-\sum_{j=0}^{\frac{N}{2}-1} \varepsilon \psi_{2 j}(x) \psi_{2 j+1}(y)+\sum_{j=0}^{\frac{N}{2}-1} \varepsilon \psi_{2 j+1}(x) \psi_{2 j}(y) \\
& =\sum_{j=0}^{\frac{N}{2}-1} \varphi_{2 j}^{(\alpha+1)}(x) \widetilde{\varphi}_{2 j}^{(\alpha+1)}(y)-\sum_{j=0}^{\frac{N}{2}-1} \varepsilon \widetilde{\varphi}_{2 j}^{(\alpha+1)}(x)\left[\varphi_{2 j}^{(\alpha+1)}(y)\right]^{\prime}
\end{aligned}
$$


Differentiating (4.5) with respect to $x$, we find,

$$
\left[\varphi_{j}^{(\alpha+1)}(x)\right]^{\prime}=\frac{1}{c_{j}^{(\alpha+1)}}\left[x\left(L_{j}^{(\alpha+1)}(x)\right)^{\prime}+\frac{\alpha+2-x}{2} L_{j}^{(\alpha+1)}(x)\right] x^{\frac{\alpha}{2}} \mathrm{e}^{-\frac{x}{2}} .
$$

Recall that the Laguerre polynomials $L_{j}^{(\alpha+1)}$ satisfy the differentiation formulas [8]

$$
\begin{gathered}
x\left(L_{j}^{(\alpha+1)}(x)\right)^{\prime}=j L_{j}^{(\alpha+1)}(x)-(j+\alpha+1) L_{j-1}^{(\alpha+1)}(x), \quad j=0,1,2, \ldots, \\
x\left(L_{j}^{(\alpha+1)}(x)\right)^{\prime}=(j+1) L_{j+1}^{(\alpha+1)}(x)-(j+\alpha+2-x) L_{j}^{(\alpha+1)}(x), \quad j=0,1,2, \ldots
\end{gathered}
$$

The sum of (4.6) and (4.7) divided by 2, gives,

$$
x\left(L_{j}^{(\alpha+1)}(x)\right)^{\prime}=\frac{j+1}{2} L_{j+1}^{(\alpha+1)}(x)-\frac{j+\alpha+1}{2} L_{j-1}^{(\alpha+1)}(x)-\frac{\alpha+2-x}{2} L_{j}^{(\alpha+1)}(x), \quad j=0,1,2, \ldots,
$$

which is the same as,

$$
x\left(L_{j}^{(\alpha+1)}(x)\right)^{\prime}+\frac{\alpha+2-x}{2} L_{j}^{(\alpha+1)}(x)=\frac{j+1}{2} L_{j+1}^{(\alpha+1)}(x)-\frac{j+\alpha+1}{2} L_{j-1}^{(\alpha+1)}(x) .
$$

Hence the derivative of $\varphi_{j}^{(\alpha+1)}(x)$ becomes,

$$
\begin{aligned}
{\left[\varphi_{j}^{(\alpha+1)}(x)\right]^{\prime} } & =\frac{1}{c_{j}^{(\alpha+1)}}\left[\frac{j+1}{2} L_{j+1}^{(\alpha+1)}(x)-\frac{j+\alpha+1}{2} L_{j-1}^{(\alpha+1)}(x)\right] x^{\frac{\alpha}{2}} \mathrm{e}^{-\frac{x}{2}} \\
& =\frac{1}{2} \sqrt{(j+1)(j+\alpha+2)} \widetilde{\varphi}_{j+1}^{(\alpha+1)}(x)-\frac{1}{2} \sqrt{j(j+\alpha+1)} \widetilde{\varphi}_{j-1}^{(\alpha+1)}(x) .
\end{aligned}
$$

So replacing $j$ by $2 j$, we see that,

$$
\left[\varphi_{2 j}^{(\alpha+1)}(y)\right]^{\prime}=\sqrt{\left(j+\frac{1}{2}\right)\left(j+\frac{\alpha+2}{2}\right)} \widetilde{\varphi}_{2 j+1}^{(\alpha+1)}(y)-\sqrt{j\left(j+\frac{\alpha+1}{2}\right)} \widetilde{\varphi}_{2 j-1}^{(\alpha+1)}(y) .
$$

Continuing, we compute the summation to give,

$$
\begin{aligned}
& \sum_{j=0}^{\frac{N}{2}-1} \varepsilon \widetilde{\varphi}_{2 j}^{(\alpha+1)}(x)\left[\varphi_{2 j}^{(\alpha+1)}(y)\right]^{\prime} \\
= & \sum_{j=0}^{\frac{N}{2}-1} \sqrt{\left(j+\frac{1}{2}\right)\left(j+\frac{\alpha+2}{2}\right)} \varepsilon \widetilde{\varphi}_{2 j}^{(\alpha+1)}(x) \widetilde{\varphi}_{2 j+1}^{(\alpha+1)}(y) \\
- & \sum_{j=1}^{\frac{N}{2}-1} \sqrt{j\left(j+\frac{\alpha+1}{2}\right)} \varepsilon \widetilde{\varphi}_{2 j}^{(\alpha+1)}(x) \widetilde{\varphi}_{2 j-1}^{(\alpha+1)}(y) \\
= & \sum_{j=1}^{\frac{N}{2}}\left[\sqrt{\left(j-\frac{1}{2}\right)\left(j+\frac{\alpha}{2}\right)} \varepsilon \widetilde{\varphi}_{2 j-2}^{(\alpha+1)}(x)-\sqrt{j\left(j+\frac{\alpha+1}{2}\right)} \varepsilon \widetilde{\varphi}_{2 j}^{(\alpha+1)}(x)\right] \widetilde{\varphi}_{2 j-1}^{(\alpha+1)}(y) \\
+ & \frac{1}{2} \sqrt{N(N+\alpha+1)} \varepsilon \widetilde{\varphi}_{N}^{(\alpha+1)}(x) \widetilde{\varphi}_{N-1}^{(\alpha+1)}(y) .
\end{aligned}
$$


From (4.8), and using Lemma 2.7, we have

$$
\begin{aligned}
\varphi_{2 j-1}^{(\alpha+1)}(x) & =\varepsilon\left[\varphi_{2 j-1}^{(\alpha+1)}(x)\right]^{\prime} \\
& =\sqrt{j\left(j+\frac{\alpha+1}{2}\right)} \varepsilon \widetilde{\varphi}_{2 j}^{(\alpha+1)}(x)-\sqrt{\left(j-\frac{1}{2}\right)\left(j+\frac{\alpha}{2}\right)} \varepsilon \widetilde{\varphi}_{2 j-2}^{(\alpha+1)}(x) .
\end{aligned}
$$

The sum simplifies to

$$
\sum_{j=0}^{\frac{N}{2}-1} \varepsilon \widetilde{\varphi}_{2 j}^{(\alpha+1)}(x)\left[\varphi_{2 j}^{(\alpha+1)}(y)\right]^{\prime}=-\sum_{j=1}^{\frac{N}{2}} \varphi_{2 j-1}^{(\alpha+1)}(x) \widetilde{\varphi}_{2 j-1}^{(\alpha+1)}(y)+\frac{1}{2} \sqrt{N(N+\alpha+1)} \varepsilon \widetilde{\varphi}_{N}^{(\alpha+1)}(x) \widetilde{\varphi}_{N-1}^{(\alpha+1)}(y) .
$$

The computations above gives a compact form for $K_{N, 1}^{(2,2)}(x, y)$ :

$$
\begin{aligned}
K_{N, 1}^{(2,2)}(x, y) & =\sum_{j=0}^{\frac{N}{2}-1} \varphi_{2 j}^{(\alpha+1)}(x) \widetilde{\varphi}_{2 j}^{(\alpha+1)}(y)+\sum_{j=1}^{\frac{N}{2}} \varphi_{2 j-1}^{(\alpha+1)}(x) \widetilde{\varphi}_{2 j-1}^{(\alpha+1)}(y)-\frac{1}{2} \sqrt{N(N+\alpha+1)} \varepsilon \widetilde{\varphi}_{N}^{(\alpha+1)}(x) \widetilde{\varphi}_{N-1}^{(\alpha+1)}(y) \\
& =\sum_{j=0}^{N-1} \varphi_{j}^{(\alpha+1)}(x) \widetilde{\varphi}_{j}^{(\alpha+1)}(y)-\frac{1}{2} \sqrt{N(N+\alpha+1)} \varepsilon \widetilde{\varphi}_{N}^{(\alpha+1)}(x) \widetilde{\varphi}_{N-1}^{(\alpha+1)}(y) \\
& =S_{N}(x, y)-\frac{1}{2} \sqrt{N(N+\alpha+1)} \varepsilon \widetilde{\varphi}_{N}^{(\alpha+1)}(x) \widetilde{\varphi}_{N-1}^{(\alpha+1)}(y),
\end{aligned}
$$

where

$$
S_{N}(x, y):=\sum_{j=0}^{N-1} \varphi_{j}^{(\alpha+1)}(x) \widetilde{\varphi}_{j}^{(\alpha+1)}(y)=-\sqrt{N(N+\alpha+1)} \frac{\varphi_{N}^{(\alpha+1)}(x) \widetilde{\varphi}_{N-1}^{(\alpha+1)}(y)-\widetilde{\varphi}_{N}^{(\alpha+1)}(y) \varphi_{N-1}^{(\alpha+1)}(x)}{x-y} .
$$

Here we have used the Christoffel-Darboux formula in the last equality.

By Theorem 4.2, we have the following theorem.

\section{Theorem 4.10.}

$$
\begin{aligned}
& {\left[G_{N}^{(1)}(f)\right]^{2} } \\
= & \operatorname{det}\left(I+S_{N}\left(f^{2}+2 f\right)-S_{N} \varepsilon f^{\prime}-S_{N} f \varepsilon f^{\prime}-\frac{1}{2} \sqrt{N(N+\alpha+1)}\left[\varepsilon \widetilde{\varphi}_{N}^{(\alpha+1)} \otimes \widetilde{\varphi}_{N-1}^{(\alpha+1)}\left(f^{2}+2 f\right)\right]\right. \\
- & \left.\frac{1}{2} \sqrt{N(N+\alpha+1)}\left(\varepsilon \widetilde{\varphi}_{N}^{(\alpha+1)} \otimes \varepsilon \widetilde{\varphi}_{N-1}^{(\alpha+1)}\right) f^{\prime}-\frac{1}{2} \sqrt{N(N+\alpha+1)}\left[\varepsilon \widetilde{\varphi}_{N}^{(\alpha+1)} \otimes \varepsilon\left(\widetilde{\varphi}_{N-1}^{(\alpha+1)} f\right)\right] f^{\prime}\right) .
\end{aligned}
$$

\subsection{Large $N$ behavior of the LOE moment generating function}

Now we consider the scaling limit of $\left[G_{N}^{(1)}(f)\right]^{2}$. We write

$$
\left[G_{N}^{(1)}(f)\right]^{2}=: \operatorname{det}(I+T),
$$


where

$$
\begin{aligned}
T: & =S_{N}\left(f^{2}+2 f\right)-S_{N} \varepsilon f^{\prime}-S_{N} f \varepsilon f^{\prime}-\frac{1}{2} \sqrt{N(N+\alpha+1)}\left[\varepsilon \widetilde{\varphi}_{N}^{(\alpha+1)} \otimes \widetilde{\varphi}_{N-1}^{(\alpha+1)}\left(f^{2}+2 f\right)\right] \\
& -\frac{1}{2} \sqrt{N(N+\alpha+1)}\left(\varepsilon \widetilde{\varphi}_{N}^{(\alpha+1)} \otimes \varepsilon \widetilde{\varphi}_{N-1}^{(\alpha+1)}\right) f^{\prime} \\
& -\frac{1}{2} \sqrt{N(N+\alpha+1)}\left[\varepsilon \widetilde{\varphi}_{N}^{(\alpha+1)} \otimes \varepsilon\left(\widetilde{\varphi}_{N-1}^{(\alpha+1)} f\right)\right] f^{\prime} .
\end{aligned}
$$

In the computations below, we replace $f(x)$ by $f(\sqrt{4 N x})$ and $f^{\prime}(x)$ by

$$
f^{\prime}(\sqrt{4 N x})=\frac{d}{\sqrt{4 N} d \sqrt{x}} f(\sqrt{4 N x}) .
$$

Similarly to the LSE case, we have the following two theorems.

\section{Theorem 4.11.}

$$
\begin{aligned}
& \lim _{N \rightarrow \infty} \frac{y}{2 N} S_{N}\left(\frac{x^{2}}{4 N}, \frac{y^{2}}{4 N}\right)=B^{(\alpha+1)}(x, y), \\
& \lim _{N \rightarrow \infty} \frac{x}{2 N} S_{N}\left(\frac{x^{2}}{4 N}, \frac{x^{2}}{4 N}\right)=B^{(\alpha+1)}(x, x),
\end{aligned}
$$

where $B^{(\alpha+1)}(x, y)$ and $B^{(\alpha+1)}(x, x)$ are given by (1.6) and (1.7) with $\alpha$ replaced by $\alpha+1$.

\section{Theorem 4.12.}

$$
\begin{gathered}
\widetilde{\varphi}_{N-1}^{(\alpha+1)}\left(\frac{x^{2}}{4 N}\right)=2 N^{\frac{1}{2}} \frac{J_{\alpha+1}(x)}{x}+O\left(N^{-\frac{3}{2}}\right), N \rightarrow \infty, \\
\varepsilon \widetilde{\varphi}_{N}^{(\alpha+1)}\left(\frac{x^{2}}{4 N}\right)=N^{-\frac{1}{2}}\left[\int_{0}^{x} J_{\alpha+1}(y) d y-1\right]+O\left(N^{-\frac{5}{2}}\right), \quad N \rightarrow \infty, \\
\varepsilon \widetilde{\varphi}_{N-1}^{(\alpha+1)}\left(\frac{x^{2}}{4 N}\right)=N^{-\frac{1}{2}} \int_{0}^{x} J_{\alpha+1}(y) d y+O\left(N^{-\frac{5}{2}}\right), N \rightarrow \infty, \\
\varepsilon\left(\widetilde{\varphi}_{N-1}^{(\alpha+1)} f\right)\left(\frac{x^{2}}{4 N}\right)=\frac{1}{2} N^{-\frac{1}{2}}\left[\int_{0}^{x} J_{\alpha+1}(y) f(y) d y-\int_{x}^{\infty} J_{\alpha+1}(y) f(y) d y\right]+O\left(N^{-\frac{5}{2}}\right), \quad N \rightarrow \infty .
\end{gathered}
$$


Using Theorem 4.11 and Theorem 4.12 to compute $\operatorname{Tr} T$ and $\operatorname{Tr} T^{2}$ as $N \rightarrow \infty$. We obtain

$$
\begin{aligned}
\operatorname{Tr} T & =\int_{0}^{\infty} B^{(\alpha+1)}(x, x)\left[f^{2}(x)+2 f(x)\right] d x \\
& -\frac{1}{2} \int_{0}^{\infty}\left[\int_{0}^{x} J_{\alpha+1}(y) d y-1\right] J_{\alpha+1}(x)\left[f^{2}(x)+2 f(x)\right] d x \\
& -\frac{1}{4 N} \int_{0}^{\infty}\left[\int_{x}^{\infty} B^{(\alpha+1)}(x, y) d y-\int_{0}^{x} B^{(\alpha+1)}(x, y) d y\right] x f^{\prime}(x) d x \\
& -\frac{1}{4 N} \int_{0}^{\infty}\left[\int_{x}^{\infty} B^{(\alpha+1)}(x, y) f(y) d y-\int_{0}^{x} B^{(\alpha+1)}(x, y) f(y) d y\right] x f^{\prime}(x) d x \\
& -\frac{1}{4 N} \int_{0}^{\infty}\left[\int_{0}^{x} J_{\alpha+1}(y) d y-1\right]\left[\int_{0}^{x} J_{\alpha+1}(y) d y\right] x f^{\prime}(x) d x \\
& -\frac{1}{8 N} \int_{0}^{\infty}\left[\int_{0}^{x} J_{\alpha+1}(y) d y-1\right]\left[\int_{0}^{x} J_{\alpha+1}(y) f(y) d y-\int_{x}^{\infty} J_{\alpha+1}(y) f(y) d y\right] x f^{\prime}(x) d x \\
& +O\left(N^{-2}\right), \quad N \rightarrow \infty,
\end{aligned}
$$


and

$\operatorname{Tr} T^{2}$

$$
\begin{aligned}
& =\int_{0}^{\infty} \int_{0}^{\infty} B^{(\alpha+1)}(x, y) B^{(\alpha+1)}(y, x)\left[f^{2}(x)+2 f(x)\right]\left[f^{2}(y)+2 f(y)\right] d x d y \\
& -\int_{0}^{\infty} \int_{0}^{\infty} B^{(\alpha+1)}(x, y) J_{\alpha+1}(x)\left[\int_{0}^{y} J_{\alpha+1}(z) d z-1\right]\left[f^{2}(x)+2 f(x)\right]\left[f^{2}(y)+2 f(y)\right] d x d y \\
& +\frac{1}{4} \int_{0}^{\infty} \int_{0}^{\infty}\left[\int_{0}^{x} J_{\alpha+1}(z) d z-1\right]\left[\int_{0}^{y} J_{\alpha+1}(z) d z-1\right] J_{\alpha+1}(x) J_{\alpha+1}(y) \\
& {\left[f^{2}(x)+2 f(x)\right]\left[f^{2}(y)+2 f(y)\right] d x d y} \\
& -\frac{1}{2 N} \int_{0}^{\infty} \int_{0}^{\infty} B^{(\alpha+1)}(x, y)\left[\int_{x}^{\infty} B^{(\alpha+1)}(y, z) d z-\int_{0}^{x} B^{(\alpha+1)}(y, z) d z\right] x f^{\prime}(x)\left[f^{2}(y)+2 f(y)\right] d x d y \\
& -\frac{1}{2 N} \int_{0}^{\infty} \int_{0}^{\infty} B^{(\alpha+1)}(x, y)\left[\int_{x}^{\infty} B^{(\alpha+1)}(y, z) f(z) d z-\int_{0}^{x} B^{(\alpha+1)}(y, z) f(z) d z\right] \\
& x f^{\prime}(x)\left[f^{2}(y)+2 f(y)\right] d x d y \\
& -\frac{1}{2 N} \int_{0}^{\infty} \int_{0}^{\infty} B^{(\alpha+1)}(x, y)\left[\int_{0}^{x} J_{\alpha+1}(z) d z\right]\left[\int_{0}^{y} J_{\alpha+1}(z) d z-1\right] x f^{\prime}(x)\left[f^{2}(y)+2 f(y)\right] d x d y \\
& -\frac{1}{4 N} \int_{0}^{\infty} \int_{0}^{\infty} B^{(\alpha+1)}(x, y)\left[\int_{0}^{x} J_{\alpha+1}(z) f(z) d z-\int_{x}^{\infty} J_{\alpha+1}(z) f(z) d z\right]\left[\int_{0}^{y} J_{\alpha+1}(z) d z-1\right] \\
& x f^{\prime}(x)\left[f^{2}(y)+2 f(y)\right] d x d y \\
& +\frac{1}{4 N} \int_{0}^{\infty} \int_{0}^{\infty}\left[\int_{x}^{\infty} B^{(\alpha+1)}(y, z) d z-\int_{0}^{x} B^{(\alpha+1)}(y, z) d z\right]\left[\int_{0}^{x} J_{\alpha+1}(z) d z-1\right] J_{\alpha+1}(y) \\
& x f^{\prime}(x)\left[f^{2}(y)+2 f(y)\right] d x d y \\
& +\frac{1}{4 N} \int_{0}^{\infty} \int_{0}^{\infty}\left[\int_{x}^{\infty} B^{(\alpha+1)}(y, z) f(z) d z-\int_{0}^{x} B^{(\alpha+1)}(y, z) f(z) d z\right]\left[\int_{0}^{x} J_{\alpha+1}(z) d z-1\right] J_{\alpha+1}(y) \\
& x f^{\prime}(x)\left[f^{2}(y)+2 f(y)\right] d x d y \\
& +\frac{1}{4 N} \int_{0}^{\infty} \int_{0}^{\infty}\left[\int_{0}^{x} J_{\alpha+1}(z) d z-1\right]\left[\int_{0}^{y} J_{\alpha+1}(z) d z-1\right]\left[\int_{0}^{x} J_{\alpha+1}(z) d z\right] J_{\alpha+1}(y) \\
& x f^{\prime}(x)\left[f^{2}(y)+2 f(y)\right] d x d y \\
& +\frac{1}{8 N} \int_{0}^{\infty} \int_{0}^{\infty}\left[\int_{0}^{x} J_{\alpha+1}(z) d z-1\right]\left[\int_{0}^{y} J_{\alpha+1}(z) d z-1\right] \\
& {\left[\int_{0}^{x} J_{\alpha+1}(z) f(z) d z-\int_{x}^{\infty} J_{\alpha+1}(z) f(z) d z\right] J_{\alpha+1}(y) x f^{\prime}(x)\left[f^{2}(y)+2 f(y)\right] d x d y} \\
& +O\left(N^{-2}\right), \quad N \rightarrow \infty \text {. }
\end{aligned}
$$

Now we want to see the mean and variance of the linear statistics $\sum_{j=1}^{N} F\left(\sqrt{4 N x_{j}}\right)$, so we need to obtain the coefficients of $\lambda$ and $\lambda^{2}$, firstly we know

$$
f(\sqrt{4 N x}) \approx-\lambda F(\sqrt{4 N x})+\frac{\lambda^{2}}{2} F^{2}(\sqrt{4 N x})
$$

then we replace $f$ with $-\lambda F+\frac{\lambda^{2}}{2} F^{2}$ in the expression of $\operatorname{Tr} T$ and $\operatorname{Tr} T^{2}$, similar to previous 
discussions, denote by $\mu_{N}^{(L O E, \alpha)}$ and $\mathcal{V}_{N}^{(L O E, \alpha)}$ the mean and variance of the linear statistics $\sum_{j=1}^{N} F\left(\sqrt{4 N x_{j}}\right)$, then we have the following theorem.

Theorem 4.13. As $N \rightarrow \infty$,

$$
\begin{aligned}
\mu_{N}^{(L O E, \alpha)} & =\mu_{N}^{(L U E, \alpha+1)}-\frac{1}{2} \int_{0}^{\infty}\left[\int_{0}^{x} J_{\alpha+1}(y) d y-1\right] J_{\alpha+1}(x) F(x) d x \\
& -\frac{1}{8 N} \int_{0}^{\infty}\left[\int_{x}^{\infty} B^{(\alpha+1)}(x, y) d y-\int_{0}^{x} B^{(\alpha+1)}(x, y) d y\right] x F^{\prime}(x) d x \\
& \quad-\frac{1}{8 N} \int_{0}^{\infty}\left[\int_{0}^{x} J_{\alpha+1}(y) d y-1\right]\left[\int_{0}^{x} J_{\alpha+1}(y) d y\right] x F^{\prime}(x) d x+O\left(N^{-2}\right), \\
\mathcal{V}_{N}^{(L O E, \alpha)}= & 2 \mathcal{V}_{N}^{(L U E, \alpha+1)}-\int_{0}^{\infty}\left[\int_{0}^{x} J_{\alpha+1}(y) d y-1\right] J_{\alpha+1}(x) F^{2}(x) d x \\
+ & 2 \int_{0}^{\infty} \int_{0}^{\infty} B^{(\alpha+1)}(x, y) J_{\alpha+1}(x)\left[\int_{0}^{y} J_{\alpha+1}(z) d z-1\right] F(x) F(y) d x d y \\
- & \frac{1}{2} \int_{0}^{\infty} \int_{0}^{\infty}\left[\int_{0}^{x} J_{\alpha+1}(z) d z-1\right]\left[\int_{0}^{y} J_{\alpha+1}(z) d z-1\right] J_{\alpha+1}(x) J_{\alpha+1}(y) F(x) F(y) d x d y \\
- & \frac{1}{4 N} \int_{0}^{\infty}\left[\int_{x}^{\infty} B^{(\alpha+1)}(x, y) d y-\int_{0}^{x} B^{(\alpha+1)}(x, y) d y\right] x F(x) F^{\prime}(x) d x \\
- & \frac{1}{4 N} \int_{0}^{\infty}\left[\int_{x}^{\infty} B^{(\alpha+1)}(x, y) F(y) d y-\int_{0}^{x} B^{(\alpha+1)}(x, y) F(y) d y\right] x F^{\prime}(x) d x \\
& -\frac{1}{4 N} \int_{0}^{\infty}\left[\int_{0}^{x} J_{\alpha+1}(y) d y-1\right]\left[\int_{0}^{x} J_{\alpha+1}(y) d y\right] x F(x) F^{\prime}(x) d x \\
& -\frac{1}{8 N} \int_{0}^{\infty}\left[\int_{0}^{x} J_{\alpha+1}(y) d y-1\right]\left[\int_{0}^{x} J_{\alpha+1}(y) F(y) d y-\int_{x}^{\infty} J_{\alpha+1}(y) F(y) d y\right] x F^{\prime}(x) d x \\
+ & \frac{1}{2 N} \int_{0}^{\infty} \int_{0}^{\infty} B^{(\alpha+1)}(x, y)\left[\int_{x}^{\infty} B^{(\alpha+1)}(y, z) d z-\int_{0}^{x} B^{(\alpha+1)}(y, z) d z\right] x F^{\prime}(x) F(y) d x d y \\
+ & \frac{1}{2 N} \int_{0}^{\infty} \int_{0}^{\infty} B^{(\alpha+1)}(x, y)\left[\int_{0}^{x} J_{\alpha+1}(z) d z\right]\left[\int_{0}^{y} J_{\alpha+1}(z) d z-1\right] x F^{\prime}(x) F(y) d x d y \\
- & \frac{1}{4 N} \int_{0}^{\infty} \int_{0}^{\infty}\left[\int_{x}^{\infty} B^{(\alpha+1)}(y, z) d z-\int_{0}^{x} B^{(\alpha+1)}(y, z) d z\right]\left[\int_{0}^{x} J_{\alpha+1}(z) d z-1\right] J_{\alpha+1}(y) \\
& x F^{\prime}(x) F(y) d x d y \\
- & \frac{1}{4 N} \int_{0}^{\infty} \int_{0}^{\infty}\left[\int_{0}^{x} J_{\alpha+1}(z) d z-1\right]\left[\int_{0}^{y} J_{\alpha+1}(z) d z-1\right]\left[\int_{0}^{x} J_{\alpha+1}(z) d z\right] J_{\alpha+1}(y) \\
& x F^{\prime}(x) F(y) d x d y+O\left(N^{-2}\right), \\
& \\
& \\
&
\end{aligned}
$$

where $\mu_{N}^{(L U E, \alpha+1)}$ and $\mathcal{V}_{N}^{(L U E, \alpha+1)}$ for $N \rightarrow \infty$ are given in (1.4) and (1.5) respectively with $\alpha$ replaced by $\alpha+1$. 


\section{Conclusion}

In this paper, we study the moment generating function of linear statistics of the form $\sum_{j=1} F\left(x_{j}\right)$, namely, the expectation value or average of

$$
\exp \left[-\lambda \sum_{j=1}^{N} F\left(x_{j}\right)\right]
$$

computed in the "background" of the symplectic and orthogonal ensembles. We then specialize to the Gaussian case, where the background weight is the normal distribution and the Laguerre case, where the background weight is the gamma distribution.

Finally, we compute the large $N$ behavior of the moment generating, where $F($.$) is suitably$ scaled and obtained the mean and variance of the corresponding linear statistics in the four cases. The more complex situation with the Jacobi background will be studied in the future, in which case the polynomials depend on two parameters $a$ and $b$.

\section{Acknowledgment}

The Authors would like to thank the Macau Science Foundation for generous support, awarding the grant FDCT/2012/A3.

\section{References}

[1] M. Adler, Spectral statistics of orthogonal and symplectic ensembles, In The Oxford Handbook of Random Matrix Theory, Oxford University Press, Oxford, 2011.

[2] E. L. Basor, C. A. Tracy, Variance calculations and the Bessel kernel, Journal of Statistical Physics 73 (1993) 415-421.

[3] E. L. Basor, Distribution functions for random variables for ensembles of positive Hermitian matrices, Commun. Math. Phys. 188 (1997) 327-350.

[4] E. L. Basor, Y. Chen, H. Widom, Determinants of Hankel Matrices, Journal of Functional Analysis 179 (2001) 214-234. 
[5] N. G. de Bruijn, On some multiple integrals involving determinants, J. Indian Math. Soc. 19 (1955) 133-151.

[6] M. Dieng, C. A. Tracy, Application of random matrix theory to multivariate statistics, In Random Matrices, Random Processes and Integrable Systems (2011) 443-507.

[7] I. Gohberg, S. Goldberg, M.A. Kaashoek, Classes of Linear Operators, vols. I, Birkhäuser Verlag, Basel, 1990.

[8] I.S. Gradshteyn, I.M. Ryzhik, Table of Integrals, Series, and Products: Seventh Edition, Elsevier(Singapore) Pte Ltd., Singapore, 2007.

[9] Y. Kuramoto, Y. Kato, Dynamics of One-Dimensional Quantum Systems: Inverse-Square Interaction Models, Cambridge University Press, Cambridge, 2009.

[10] N.N. Lebedev, Special Functions and Their Applications, Dover Publications, INC., New York, 1972.

[11] M. L. Mehta, Random Matrices:Third Edition, Elsevier(Singapore) Pte Ltd., Singapore, 2006.

[12] A. Selberg, Bemerkninger om et multiplet integral, Norsk Matematisk Tidsskrift 26 (1944) $71-78$.

[13] G. Szegö, Orthogonal Polynomials: Fourth Edition, American Mathematical Society, Providence, RI, 1975.

[14] C. A. Tracy, H. Widom, On orthogonal and symplectic matrix ensembles, Commun. Math. Phys. 177 (1996) 727-754.

[15] C. A. Tracy, H. Widom, Correlation functions, cluster functions and spacing distributions for random matrices, Journal of Statistical Physics 92 (1998) 809-835.

[16] H. Widom, On the relation between orthogonal, symplectic and unitary matrix ensembles, Journal of Statistical Physics 94 (1999) 347-363. 